\title{
DigITALCOMMONS
}

$@$ WAYNESTATE -

Wayne State University

Wayne State University Dissertations

$1-1-1998$

\section{The relationship of cognitive and emotional coping to adjustment to chronic myofascial pain}

Julie A. Smith

Follow this and additional works at: http://digitalcommons.wayne.edu/oa_dissertations

\section{Recommended Citation}

Smith, Julie A., "The relationship of cognitive and emotional coping to adjustment to chronic myofascial pain" (1998). Wayne State University Dissertations. Paper 1216. 
THE RELATIONSHIP OF COGNITIVE AND EMOTIONAL COPING TO ADJUSTMENT TO CHRONIC MYOFASCIAL PAIN

\author{
by \\ JULIE ANN SMITH \\ DISSERTATION
}

Submitted to the Graduate School

of Wayne State University

Detroit, Michigan

in partial fulfillment of the requirements

for the degree of

DOCTOR OF PHILOSOPHY

1998

MAJOR: PSYCHOLOGY (Clinical)

Approved by: 4 L temley 7/-20/98

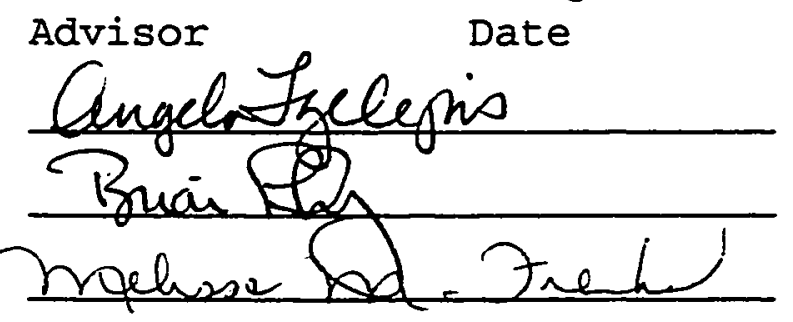




\section{ACKNOWLEDGEMENTS}

First and foremost, I would like to express my deepest appreciation to Dr. Mark Lumley, for all of his tireless efforts in helping me accomplish this goal and for never failing to match my pace along the way. Thank you so much for your support, encouragement, and dedication to helping me along the way.

Thanks to my committee members, Dr. Brian Lakey, Dr. Angela Tzelepis and Dr. Melissa Franks for their support and helpful feedback. Thanks also to Jason Nupp for his help recruiting subjects.

Eternal thanks to Dr. David Longo, the sweetest, most genuine, albeit "asocial" guy I know. Thanks for providing more professional support than I ever thought possible. Your quiet way of doing for others and asking nothing in return does not go unnoticed and is greatly appreciated. Thanks also for sharing your blue-collar sense of humor and depressive pessimism at all the right moments.

Overdue thanks to the strong women who have been role models at different stages in my life: the first, Monica Domagala, who taught me at an early age about what it means to be a feminist and who unknowingly ignited the initial spark; Nancy Ryan, who provided spiritual and emotional nurturance during a time of great need; and Lori KleinShapiro, who taught me countless, invaluable life lessons and helped me understand my "process" with more clarity. 
Heartfelt thanks to my dearest friends who always had faith in me even when I did not, and who always have been my strongest supporters in this and every other aspect of my life: Dr. Jana Brittain Drew, Kim Sirl, Michelle Todd, Dr. Caren DeBernardo, and Noel Dean Atkins.

Many thanks to my family members who have provided real and practical support through it all, especially, my parents, Sherman and Marlene Smith; my brother and his family, Charlie, Lynnette, and zachary Smith; my maternal grandparents, Ed and Frances Jourdan Sr.; and my aunt, Donna Jourdan. 
PAGE

ACKNOWLEDGEMENTS .........................

LIST OF TABLES ....................... vii

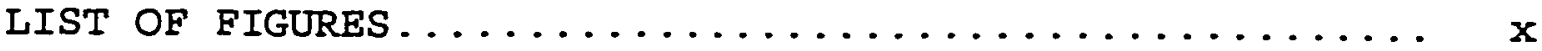

I. GENERAL INTRODUCTION AND LITERATURE REVIEW

A. CHRONIC PAIN......................... 1

B. MYOFASCIAL PAIN SYNDROMES............... 5

C. THE INTERPLAY OF PSYCHOLOGICAL AND PHYSIOLOGICAL FACTORS IN THE CHRONIC PAIN EXPERIENCE....... 16

D. COGNITIVE FACTORS IN COPING AND ADJUSTMENT TO CHRONIC RAIN....................... 20

E. EMOTIONAL EACTORS IN COPING AND ADJUSTMENT TO CHRONIC RAIN....................... 34

F. SUMMARY AND SRECIFIC AIMS ............. 57

G. HYPOTHESES ....................... 59

II. METHODOLOGY

A. PARTICIPANTS ..................... 61

B. PROCEDURES ......................... 64

C. MEASURES ......................... 67

III. RESULTS

A. OVERVIEW OF RESULTS AND ANALYSES ........... 84

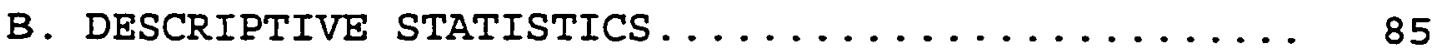

C. DATA REDUCTION..................... 89

D. RELATIONSHIPS AMONG PREDICTORS ............ 106

E. RELATIONSHIPS AMONG PREDICTORS AND OUTCOMES.... 110

F. PARTIAL CORRELATIONS ................. 113

G. MULTIPLE REGRESSION ANALYSES ............ 116 
H. THE RELATIONSHIP BETWEEN STRESS AND COPING.... 131

I. EXPLORATORY MODERATOR ANALYSES ............. 132

IV. DISCUSSION

A. REDUNDANCY AMONG MEASURES OF CONSTRUCTS . . . . . 137

B. COGNITIVE FACTORS AND ADJUSTMENT........... 143

C. EMOTIONAL FACTOR AND ADJUSTMENT............ 148

D. UNIQUE PREDICTIVE ABILITIES OF EMOTIONAL COPING 150

E. UNIQUE PREDICTIVE ABILITIES OF COGNITIVE COPING 151

F. RELATIONSHIPS BETWEEN LIFE STRESS, COPING, AND

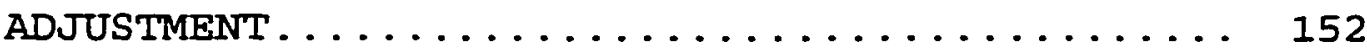

G. ADDITIONAL FINDINGS................. 155

H. IMPLICATIONS ...................... 162

I. LIMITATIONS OF THE STUDY.............. 166

J. FUTURE RESEARCH DIRECTIONS ............. 171

V. APPENDICES

A. INFORMED CONSENT . . ................. 175

B. PATIENT INFORMATION FORM.............. 177

C. VANDERBILT MULTIDIMENSIONAL PAIN COPING INVENTORY

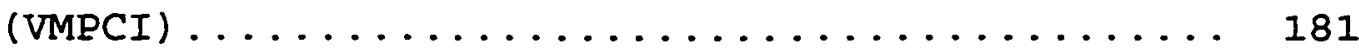

D. CHRONIC PAIN SELF-EFFICACY SCALE (CPSS) ....... 186

E. SELF-CONTROL SCALE.................... 192

F. TWENTY-ITEM TORONTO ALEXITHYMIA SCALE (TAS-20) • 194

G. EMOTIONAL APPROACH COPING SCALE........... 196

H. TRAIT META-MOOD SCALE - 30-ITEM FORM (TMMS-30). 198

I. LIFE EVENTS CHECKLIST................ 201

J. CATASTRORHIZING SCAIE................. 204

K. EMOTIONAL APPROACH COPING SCALE - PAIN VERSION. 207

L. PAIN RATING INDEX (PRI) OF THE MCGILL PAIN 


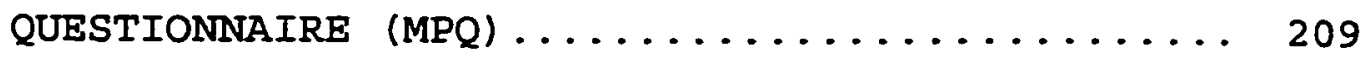

M. VISUAL ANALOGUE SCALES (VAS) ............. 211

N. INTEREERENCE SCALE ................... 213

O. CENTER FOR EPIDEMIOLOGIC STUDIES - DEPRESSION

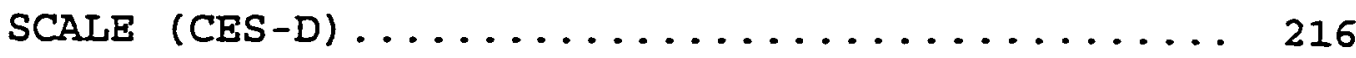

P. POSITIVE AND NEGATIVE AFFECT SCHEDULE EXPANDED FORM $($ PANAS-X) ............... 218

VI. REFERENCES ....................... 220

VII. ABSTRACT........................ 228

VIII. AUTOBIOGRAPHICAL STATEMENT............... 230 


\section{IIST OF TABLES}

TABLE

PAGE

1. Demographic Information.................. 63

2. Medical History Information................. 65

3. Pain Therapy Information................. 66

4. Descriptive Statistics for Predictors........... 86

5. Descriptive Statistics for Outcomes............ 88

6. Zero-Order Correlations among Cognitive Variables.. 90

7. Zero-Order Correlations among Emotional Variables.. 93

8. Zero-Order Correlations among Outcomes.......... 95

9. Oblimin-Rotated Loadings of the Cognitive

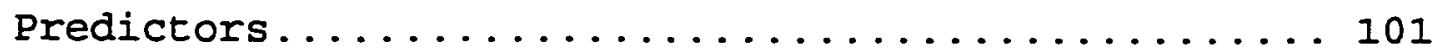

10. Factor Loadings of the Emotional Predictors...... 103

11. Oblimin-Rotated Loadings of the Cognitive and

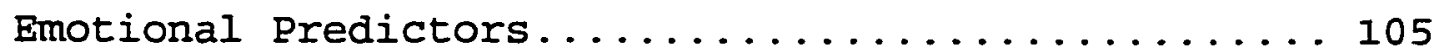

12. Zero-Order Correlations among Predictors....... 107

13. Zero-Order Correlations of Predictors with Outcome Variables................................ 11

14. Partial Correlations of Predictors with Outcomes, Controlling for Sex, Age, Education, and Pain

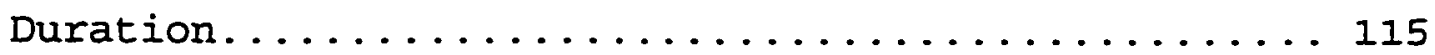

15. Hierarchical Regression of Sensory Pain on Self-Efficacy, Controlling for Cognitive Coping and Sociodemographic Variables................. 117

16. Hierarchical Regression of Affective Pain on SelfEfficacy, Controlling for Cognitive Coping and Sociodemographic variables................. 118 
17. Hierarchical Regression of Physical Impairment on Self-Efficacy, Controlling for Cognitive Coping and Sociodemographic Variables................ 119

18. Hierarchical Regression of Depression on SelfEfficacy, Controlling for Cognitive Coping and Sociodemographic Variables.................. 120

19. Hierarchical Regression of Physical Impairment on Cognitive Coping, Controlling for Self-Efficacy and Sociodemographic variables.................. 122

20. Hierarchical Regression of Affective Pain on Emotional Coping, Controlling for Cognitive Factors and Sociodemographic Variables............... 123

21. Hierarchical Regression of Depression on Emotional Coping, Controlling for Cognitive Factors and Sociodemographic variables.................. 124

22. Hierarchical Regression of Sensory Pain on Emotional Coping, Controlling for Cognitive coping and Sociodemographic variables................. 126

23. Hierarchical Regression of Sensory Pain on Cognitive Factors, Controlling for Emotional coping and Sociodemographic variables................. 127

24. Hierarchical Regression of Affective Pain on Cognitive Factors, Controlling for Emotional Coping and Sociodemographic variables................. 128

25. Hierarchical Regression of Physical Impairment on Cognitive Factors, Controlling for Emotional Coping and Sociodemographic Variables............... 129 
26. Hierarchical Regression of Depression on Cognitive Factors, Controlling for Emotional Coping and Sociodemographic variables................ 130 


\section{LIST OF FIGURES}

1. Tender Point Sites in Fibromyalgia............ 6

2. Myofascial Trigger Points and Pain Reference Patterns.......................... 8

3. Mechanisms in Myofascial Pain syndrome........... 9 
The International Association for the study of Pain (IASP) has defined pain as "an unpleasant sensory and emotional experience associated with actual or potential tissue damage, or described in terms of such damage (Merskey \& Bogduk, 1994, p. 210)." This widely accepted definition of pain acknowledges that pain is a multidimensional phenomenon that includes both sensory and affective components. As such, pain is always a subjective experience with multiple physical, psychological, and social factors influencing one's perceptions of pain intensity and pain tolerance, and one's response to the pain experience.

\section{Chronic pain}

Chronic pain should be differentiated from acute pain. Acute pain is adaptive and functional in that it warns an individual that injury or tissue damage may occur or has occurred, and it acts as a signal that appropriate action, such as eliminating the source of pain, or seeking respite or medical treatment should be taken. Acute pain also can lead to temporary limitations in activity which prevent further damage from occurring. When the signals of acute pain are attended to and appropriate measures are taken, acute pain typically resolves within hours or days (Bonica, 1990a).

Unlike acute pain, chronic pain is usually defined as pain that persists for six months or more, long after the time when somatic healing normally takes place. According to Bonica (1990b), 
Chronic pain is caused by chronic pathologic processes in somatic structures or viscera, or by prolonged and sometimes permanent dysfunction of the peripheral or central nervous system or both, and, in contrast to acute pain, it can also be caused primarily by psychologic mechanisms and/or environmental factors ( $p$. 181).

Chronic pain serves no beneficial function and is resistant to medical intervention. Chronic pain severity may vary over time and may or may not correspond to a recognizable active pathophysiologic or pathoanatomic process (Bonica, 1990a; Chapman, 1991). Chronic pain is particularly problematic because of the profound physical, emotional, social, and economic stresses that it may place on affected patients, thereby decreasing their overall quality of life (Bonica, 1990a; Bonica, 1990b; Mims, 1989).

Chronic pain is a major health and economic problem in the United States. Researchers have estimated that onethird of all Americans suffer from chronic pain (Bonica, 1990b). Many of these individuals have difficulty maintaining gainful employment due to the physical and psychological limitations of their pain (Mims, 1989). In excess of 400 million days of work were lost in 1986 due to chronic pain. In 1985, it was estimated that pain cost over $\$ 65$ billion in lost work productivity in the United States, with approximately two-thirds of this amount attributable to chronic pain (Bonica, 1990b). 
Chronic pain patients have been found to evidence numerous somatic symptoms in response to their pain conditions. These symptoms can include sleep and appetite disturbance, fatigue, constipation, increased irritability, decreased libido and sexual activity, psychomotor retardation, and lowered pain tolerance. Some chronic pain researchers believe that these vegetative signs may be due to depletion of serotonin and endorphins associated with chronic pain. This may account for the reversal of some of these symptoms when serotonergic agonist antidepressants are administered (Bonica, 1990b).

The most frequent complaint associated with chronic pain is difficulty initiating and maintaining sleep. Many patients also report consistently decreased energy, both in response to the lack of sleep and the continuous experience of pain. Appetite changes also are common with some patients experiencing loss of appetite and weight and others experiencing weight gain due to increased eating and inactivity. Persistent pain combined with the above factors can lead to decreased pain tolerance, even with relatively minor subsequent injuries, and to mood disturbance, often accompanied by irritability rather than sadness (Bonica, 1990b).

Chronic pain is associated with emotional distress including depression and anxiety. Prevalence rates for depression are consistently higher for chronic pain patients than for the general population. Romano and Turner (1985) 
reviewed the literature concerning the relationship between pain and depression. These authors concluded that depression and chronic pain develop simultaneously for approximately $50 \%$ of chronic pain patients, with $40 \%$ becoming depressed after experiencing pain for six months or more. The remaining $10 \%$ develop pain subsequent to depression. The majority of chronic pain patients do not have a history of premorbid emotional disturbance (Brown, 1990). Yet, both depression and anxiety are manifestations of adjustment in chronic pain patients. Level of depression has been found to correlate with pain intensity (Philips, 1988). Further, depressed pain patients evidence a higher treatment drop-out rate than non-depressed pain patients. Anxiety also has been associated with high pain intensity. In addition, anxiety about pain and about further injury can contribute to behavioral avoidance and increased pain behavior (Lethem, Slade, Troupe, \& Bentley, 1983). Overall, emotional distress has been found to be an important correlate of quality of life and treatment completion in chronic pain patients. Patients who are more depressed and anxious demonstrate more pain behavior, report higher pain intensity, and have a tendency to drop out of treatment than those with lower levels of emotional distress.

Chronic pain can have a negative effect on family and other social relationships due to factors such as increased irritability and depression, altered personal and occupational roles, and decreased income and independence 
for the chronic pain patient. Along with increased problems with family and friends, chronic pain patients may withdraw from social activities and become increasingly isolated. Chronic pain patients may become physically and emotionally disabled by pain to the extent that they are no longer able to maintain gainful employment. Patients who receive disability payments frequently experience economic strain and reduced standard of living, as well as decreased selfesteem and social status (Bonica, 1990b; Mims, 1989).

Myofascial pain Syndromes

Myofascial pain is one of the primary types of chronic pain seen in general practice and chronic pain clinics in the United States. Myofascial pain syndromes comprise a large group of separate but interrelated muscle disorders that are ranked among the most frequent causes of persistent and disabling pain (Simons, 1989; Sola \& Bonica, 1990).

Myofascial pain syndromes include both diffuse pain syndromes, which involve several muscles, and specific pain syndromes, which affect a single muscle. Fibromyalgia is considered a diffuse myofascial pain syndrome. Fibromyalgia is characterized by widespread pain which is experienced in multiple predictable, and usually symmetrically distributed, tender points in muscles and/or their investing connective tissue (e.g. tendons that join muscles, etc.). On digital palpation, 11 out of 18 potential tender point sites must be present for clinical diagnosis of fibromyalgia (see Figure 1). Stiffness, fatigue, and sleep disturbance typically 


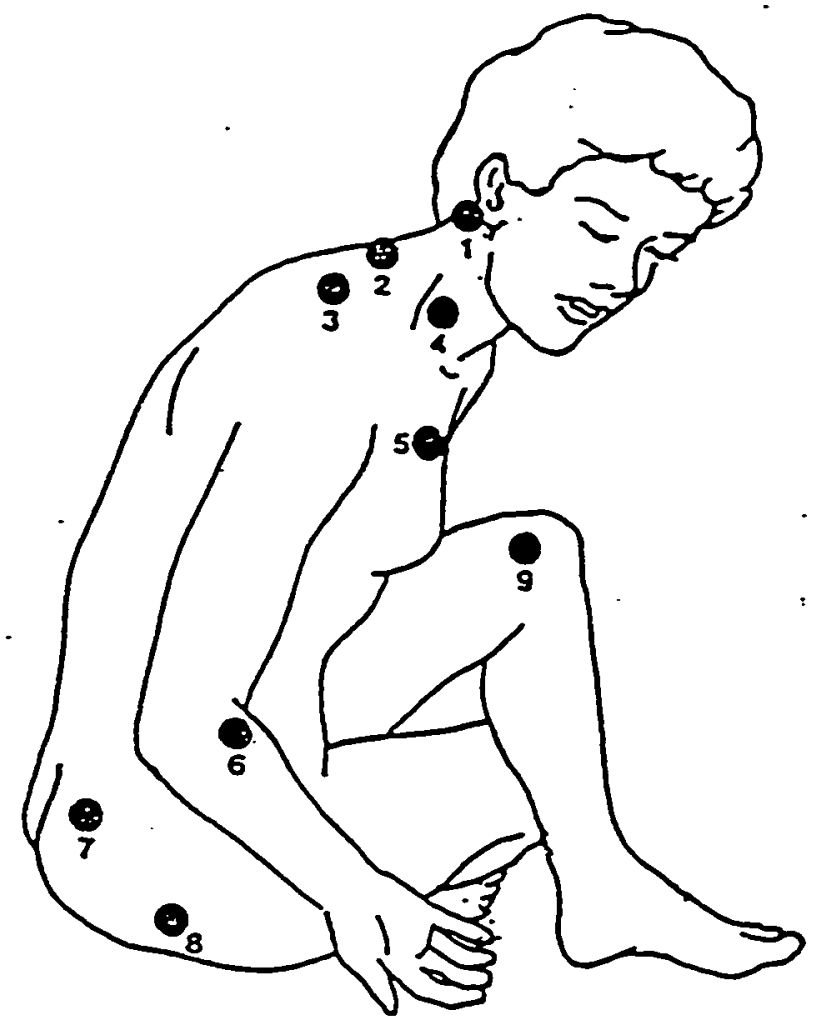

Figure 1. Tender point sites in fibromyalgia. 
accompany this syndrome. Primary fibromyalgia is uncommon, while concomitant fibromyalgia occurs in association with another musculoskeletal condition, where it typically acts to intensity the pain of the associated condition. Fibromyalgia is always considered a chronic condition (Merskey \& Bogduk, 1994).

Specific myofascial pain syndromes begin as a focal disorder in one muscle; however, a chronic myofascial pain syndrome that proliferates over time due to perpetuating factors is often virtually indistinguishable from a diffuse myofascial pain syndrome (Simons \& Simons, 1990). The pain of both specific and diffuse syndromes have the same qualities (Merskey \& Bogduk, 1994).

Patients with myofascial pain syndromes present with hypersensitive points or trigger points within one or more muscles and/or the investing connective tissue (Sola \& Bonica, 1990). Myofascial trigger points are located within palpable taut bands of muscle. They produce local tenderness, distributions of persistent referred pain (pain felt in a part of the body that is served by the same nerve or nerve root but is at some distance from its cause), and deep hyperalgesia or tenderness in the reference zone (see Figures 2 and 3) (Simons, 1989; Sola \& Bonica, 1990). When trigger points are palpated, a distinct pain response or jump sign is elicited. Associated symptoms of myofascial pain syndromes include muscle spasm, tenderness, stiffness, limited range of motion, weakness, fatigue, and occasionally 
Figure 2 .

A. Pain reference patterns of trigger areas in the upper portion of infraspinatus muscle as. mapped in 193 patients with shoulder pain. The black are is called the "essential" reference zone because $100 \%$ of the subjects felt pain there; the shaded and stippled areas are collectively labeled the "spillover" reference zone because not all patients felt pain in these areas.

A.

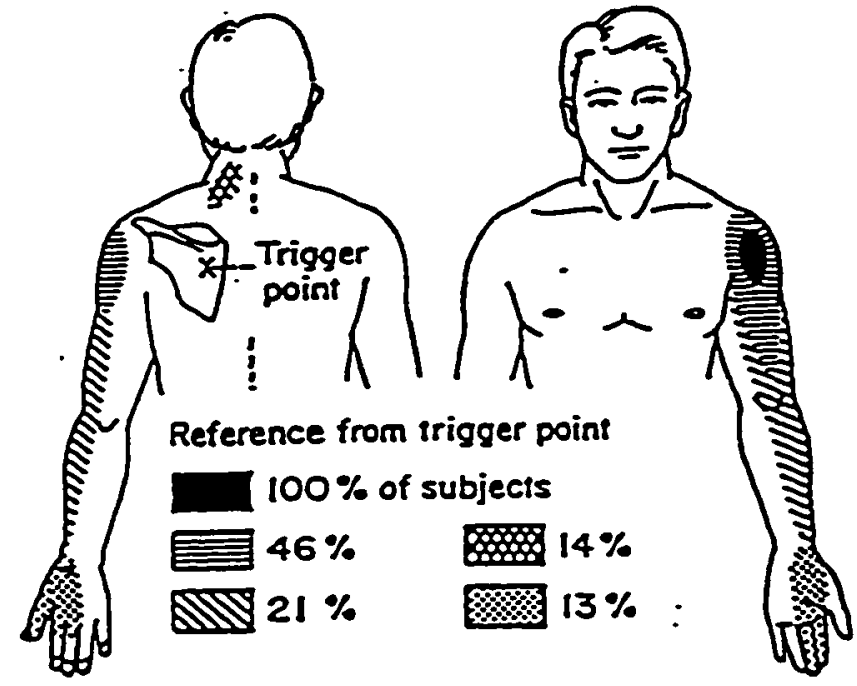

B.

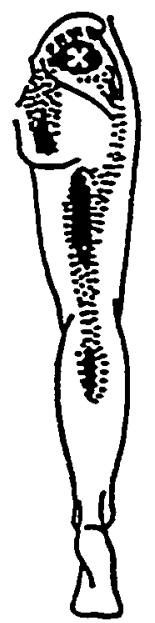

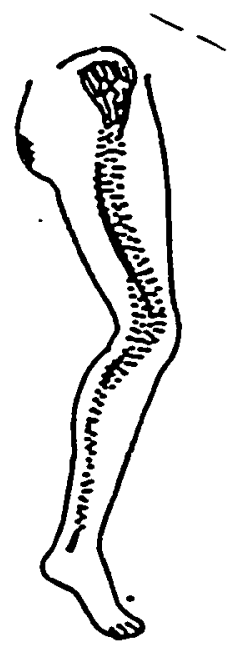

B. Gluteus medius trigger point (one of the most powerful trigger points in the body, with its local pain and referral pattern to the thigh and legs), and tensor fascia lata trigger point and characteristic hip pain and lateral thigh and leg reference zones.

C.
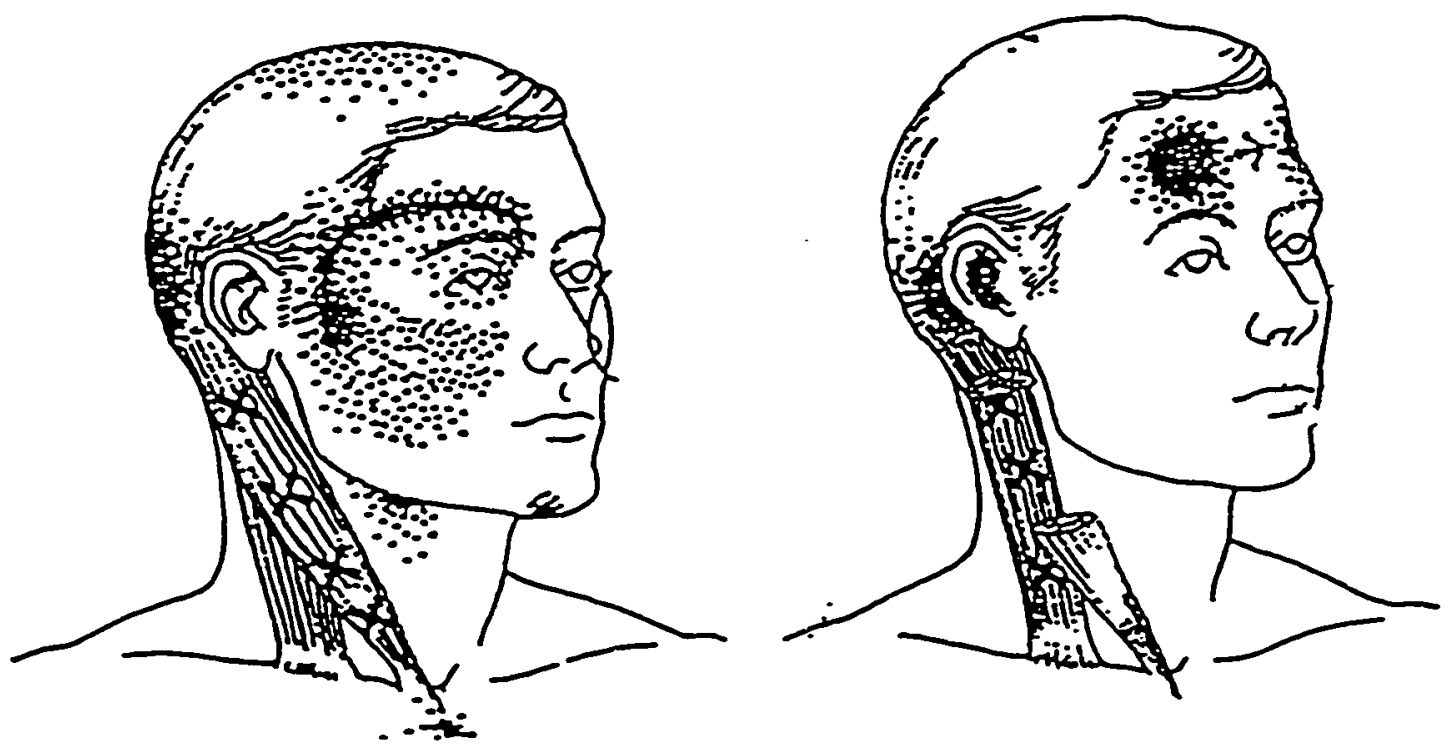

C. Referred pain patterns (solid black shows essential zones and stippling shows the spillover area with location of corresponding trigger points (X's) in the right sternocleidomastoid muscle. 
Figure 3.

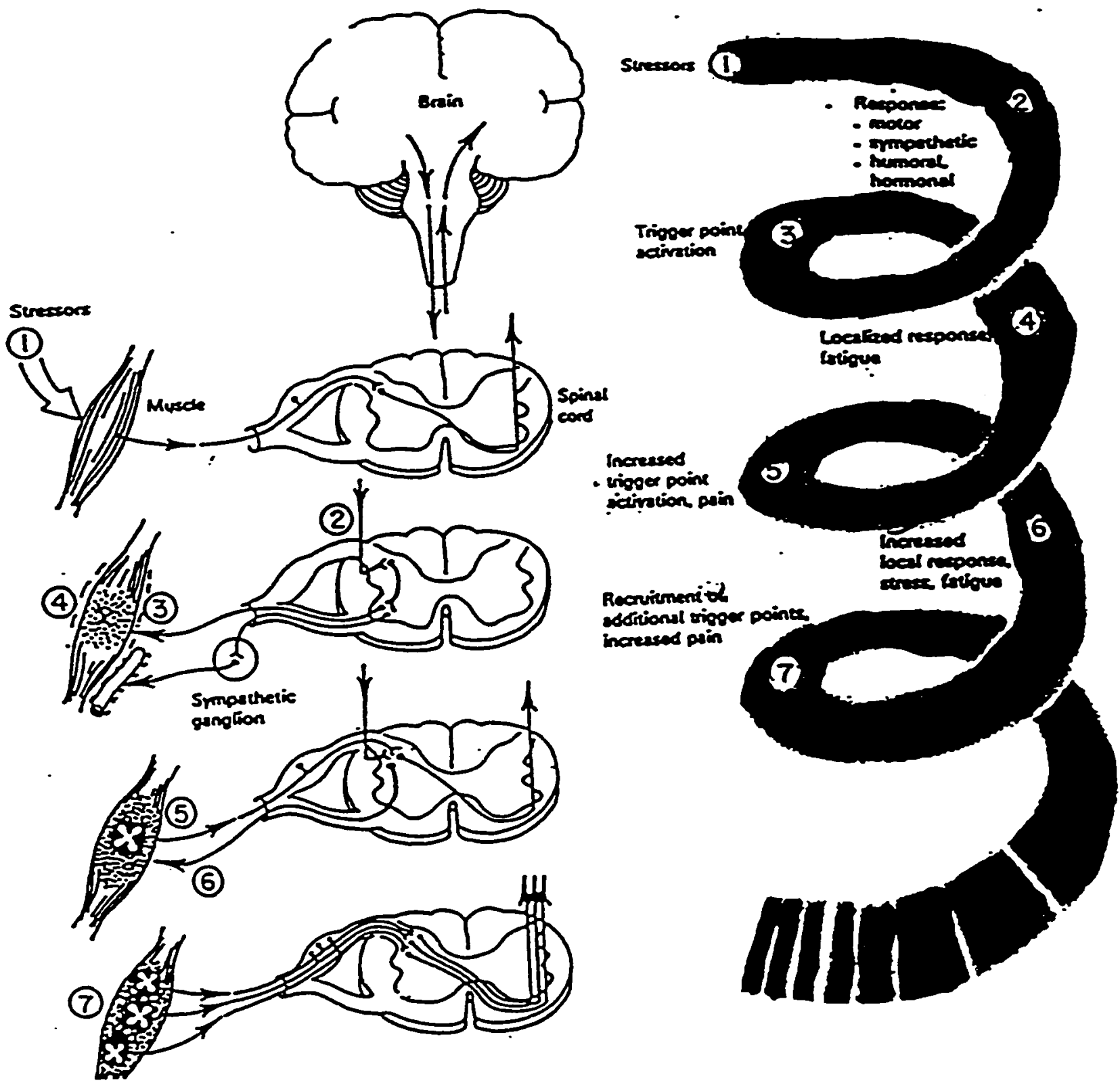

Mechanisms in myofascial pain syndrome. (1) The individual, subjected to the physical and emotional stresses of daily living responds with defense mechanisms that include various physiologic changes, such as splinting and bracing of muscles, vasomotor changes, increased sympathetic discharge, and hormonal, and other humoral changes in the plasma and extraceliular fluids. $(2,3)$ A particular point in a braced, stressed muscle or fascia that is more sensitive than the surrounding tissue - perhaps due to previous injury or genetic mandate - fatigues and begins to signal its distress to the central nervous system. (4) A number of responses are possible. The most readily understood involves the motor 
reflexes. Various muscles associated with the trigger point become tense and begin to fatigue. Sympathetic responses lead to vasomotor changes within and around the trigger point. Local ischemia following vasoconstriction or increased vascular permeability following vasodilation can lead to changes in the extracellular environment of the affected cells, release of algesic agents (bradykins, prostagladins), osmotic changes, and pH change, all of which can increase the sensitivity or activity of the nociceptors in the area. Sympathetic activity can also cause smooth muscle contraction in the vicinity of nociceptors, thus increasing their

activity. $(5,6)$ Increased nociceptor input contributes to the cycle by increasing motor and sympathetic activity, which in turn leads to increased pain. The pain is shadowed by growing fatigue, which adds an overall mood of distress to the patient's situation and feeds back to the cycle. (7) As tense muscles in the affected area begin to fatigue in an environment of sympathetic stimulation and local biochemical change, latent trigger points within these muscles also begin to fire, thus adding to the positive feedback cycle and spreading the pain to these adjacent muscle groups. Finally, the stress of pain and fatigue, coupled with both increased muscle tension and sympathetic tone throughout the body (conceivably with ipsilateral emphasis through the sympathetic chain), leads to flare-ups or trigger points in other muscles remote from the initial area of pain. Modified from Sola, A.E.: Myofascial trigger point therapy. Med Times, 110:70-5. 1982 . 
autonomic dysfunction (Simons, 1989; Sola \& Bonica, 1990).

There is some evidence to suggest that myofascial pain syndromes may affect the head and neck, shoulder area, and lumbar and low back regions more frequently than other regions of the body (Sola \& Bonica, 1990). Nonetheless, trigger points can occur in any skeletal muscle and can be active or latent. Active trigger points can be differentiated from latent trigger points by the presence of spontaneous pain that occurs both at rest and with any motion that stretches or overloads the muscle. Iatent trigger points are less irritable than active trigger points and only cause local tenderness and referred pain when pressure is applied directly to the trigger points themselves (Simons, 1989).

Some researchers have argued that the tender points found in fibromyalgia patients do not refer pain, unlike the trigger points found in patients with specific myofascial pain patients. Simons and Simons (1990) cite a scandinavian study in which the referred pain criterion was examined more closely. In this study, the majority of patients with fibromyalgia and specific myofascial pain syndromes were found to have both hypersensitive points that referred pain, as well as those that did not. Only $16 \%$ of primary fibromyalgia patients were found to have only tender points that did not refer pain. These authors suggest that the majority of studies of patients with myofascial pain syndromes have included a mixed population of patients with 
both fibromyalgia and chronic myofascial pain syndromes. The precise cause of myofascial pain syndromes is unknown; however, trauma to myofascial structures, strain, and acute or chronic muscle overload have been implicated as primary causes of myofascial pain syndromes (Simons, 1989; Sola \& Bonica, 1990). Trigger points can develop after acute injury in some individuals. There is some evidence to suggest that trigger points may be more likely to develop in less well-conditioned individuals who engage in occasional periods of intense physical activity than in those who regularly exercise their muscles (Sola \& Bonica, 1991). Thus, overusing unconditioned muscles might lead to an acute episode of myofascial pain and the development of one or more trigger points (Sola \& Bonica, 1990).

Trigger points may remain latent in a muscle for long periods of time, contributing to an injury pool and producing mild symptoms such as a slight decrease in range of motion. After an injury pool develops in a given muscle, subsequent injuries or repeated microtraumas caused by repetitive movements can lead to the activation of a trigger point and its associated zone of referred pain (Sola \& Bonica, 1990). When perpetuating factors, such as psychological or postural stress, are present, or a significant amount of noxious stimulation occurs, trigger points become hypersensitive and secondary trigger points tend to develop within the muscle group, further and further away from the initial injury site. Along with these 
changes, a cycle of increasing motor and/or sympathetic activity can be established which produces increased pain that is disproportionate to the original injury and persists after the initial stress or injury is resolved (see Figure 3) (Simons, 1989; Sola \& Bonica, 1990).

Some studies have suggested that prolonged myofascial pain symptoms and disability are caused by psychological factors. An alternate view is that trigger points are a physiological mechanism through which a psychophysiological process manifests and perpetuates itself. Sola and Bonica (1990) state,

Regardless of the causative factor, i.e., whether a trigger point is the result of injury, psychologic stress, or environmental factors, once it has formed, its own hypersensitivity is sufficient to generate progressively greater responses that are painful and physically limiting (p. 358).

Simons and Simons (1990) have described numerous systemic and mechanical perpetuating factors that can convert an acute single-muscle myofascial pain syndrome into a chronic myofascial pain syndrome. Systemic factors increase the irritability of skeletal muscles, making them more vulnerable to the development of initial and secondary trigger points. Systemic factors that have been implicated include enzyme dysfunction due to nutritional inadequacy, metabolic and endocrine dysfunction, chronic infection or infestation, and psychological stress. Mechanical factors 
can overload and aggravate existing trigger points in specific muscles. Mechanical factors include anatomic variations such as a short upper arms, seated and standing postural stress, and vocational stress that overloads a muscle through sustained use or repetitive motions. It has been noted that the presence of perpetuating factors often renders treatment of myofascial syndromes ineffective while correction of a significant perpetuating factor reduces the irritability of muscles and increases responsiveness to specific myofascial therapy (Simons \& Simons, 1990).

Diagnosis of myofascial pain syndromes typically involves a detailed history of the pain problem, a general physical examination, and a systematic search for tight bands of muscle and trigger points and zones by manual palpation or pressure threshold measurement. Laboratory tests and imaging are useful in identifying perpetuating factors. Thermography also can be used to document trigger points. Thermographic patterns found in patients with specific myofascial pain syndromes are indistinguishable from those found in patients with fibromyalgia (Simons, 1989; Sola \& Bonica, 1990).

Optimal treatment of chronic myofascial pain syndromes is holistic and interdisciplinary in nature often involving a team that includes a physician, a clinical psychologist or other mental health professional, and a physical therapist. Attempts are made to manage the negative physical, psychological, and social effects associated with chronic 
pain syndromes. In patients with chronic myofascial pain syndromes, perpetuating factors also need to be identified and resolved.

Specific myofascial pain therapy typically involves attempting to interrupt the pain cycle by eliminating individual trigger points. Frequently used treatments include trigger point injections with local anesthetic or saline; stretch and spray techniques with vapocoolants; sympathetic blocks; intensive physical therapy including components such as massage, heat and/or ice, and transcutaneous electrical nerve stimulation (TENS); and medications including NSAIDS, muscle relaxants, and antidepressants (Simons, 1989; Sola \& Bonica, 1990).

Psychological intervention generally has been cognitive-behavioral in nature with an emphasis on changing pain-related cognitions and teaching patients adaptive coping skills, pacing behaviors, and pain management techniques (Turk \& Rudy, 1989). Patient education also should factor into treatment so that patients learn about their specific pain patterns and can avoid movements or activities that overload the muscles.

Treatment for patients with chronic myofascial pain syndromes often must be ongoing in nature in order to circumvent the Iimitations and impairment of functioning which can be associated with persistent pain. Evidence suggests that even when active trigger points are deactivated, latent trigger points remain that are extremely 
vulnerable to reactivation (Simons, 1989). Treatment of myofascial pain syndromes that have occurred secondary to ligmental injury, such as from automobile accidents, responds less well to standard treatment and appears to have a worse prognosis (Sola \& Bonica, 1990).

It is important to point out that not all patients suffering from chronic myofascial pain syndromes evidence poor functioning and impairment. As will be further discussed in more detail, chronic pain patients vary widely in their ability to cope with and adjust to the various physical, psychological, and social stressors facing them. While some patients manifest adjustment problems including debilitating pain, affective disturbance, and physical dysfunction, others continue to live fulfilling and satisfying lives despite their chronic pain condition. The Interplay of Psychological and physiological Eactors in the Chronic Pain Experience

Recent models of chronic pain are guided by the biopsychosocial model which acknowledges the interaction between biological, environmental, emotional, and cognitive factors in the pain experience. Fortunately, this increased understanding of the interaction between physical and psychological factors has helped to dispel the dualistic notion that pain is either solely functional or solely organic.

There is an abundance of evidence that physiological changes can have psychological effects. Both acute and 
chronic medical problems have been implicated in the development of behavioral and psychological symptoms. For example, disturbances in thyroid hormone functioning have been found to result in hallucinations, paranoia, delusions, anxiety, and depression (Hall \& Beresford, 1986).

Similarly, delirium can result from such diverse medical problems as metabolic or fluid and electrolyte imbalance, cerebrovascular accident, and renal failure (Horvath, 1986).

In parallel fashion, research has shown that psychological processes can have physiological effects. For example, biofeedback, relaxation, and meditation have been found to alter physiological processes by altering cognitive, emotional and behavioral responses (Blumenthal, 1986). In addition, there is a considerable amount of evidence demonstrating that anxiety and a variety of stressors can impair immune system functioning and can contribute to the onset, maintenance, and exacerbation of numerous health problems. For example, psychosocial factors appear to contribute to the onset of multiple autoimmune disorders such as rheumatoid arthritis and Graves' disease (Stein, Schleifer, \& Keller, 1986).

The interplay between psychological and physiological factors is evident when examining the role of stress in the experience of chronic pain. In chronic pain, stress and pain exist in a cycle. Psychological stress has been found to increase muscle activity and lead to physiologic changes that can exacerbate and maintain existing pain problems. In 
particular, psychological stress can serve as a perpetuating factor in the transformation of an acute myofascial pain problem into a chronic myofascial pain syndrome (simons \& Simons, 1989; Sola \& Bonica, 1990; Sternbach, 1990). Further, if the underlying psychological stress perpetuating pain is not resolved, therapeutic interventions can be undermined.

Ultimately, in the experience of chronic pain, one's characteristic response to emotional stress may be more important than the experience of stress itself. The psychophysiology literature suggests that some individuals have a tendency to primarily respond to stressful situations with heightened muscle tension, and have an impaired or slow muscle relaxation response (Sternbach, 1990). When stressful conditions persist or occur frequently, these phasic responses can become tonic with muscle contraction spreading and stimulating additional muscle contraction in adjacent muscles thereby contributing to a state of chronic pain (Sternbach, 1990). Thus, even when myofascial pain originates from injury or physical trauma, both physiological and psychological events, and one's response to them, can set in motion a chain of events that results in intense pain that continues long after the original injury. Such a chain of events has been clearly delineated in the case of chronic myofascial pain (see Figure 3). Chronic muscle tension, or hyperarousal, has been found to aggravate and activate already existing trigger points which in turn 
leads to the development of additional trigger points. Specifically, this occurs when the increased sympathetic activity produces vasomotor changes leading to local ischemia (oxygen-depletion) and the release of algogenic (pain-eliciting) substances such as bradykinins and prostaglandins, which increase sensitivity to pain. The experience of increased pain then acts as a further stressor, causing increased fatigue and emotional distress, thereby creating a vicious cycle of pain and stress (Bonica, 1990b; Chapman, 1991; Sola \& Bonica, 1990).

Once a chronic pain condition is established, the experience of pain itself becomes a stressor with which one must cope. The majority of chronic pain studies and treatment protocols assume that chronic pain is the primary stressor in patients' lives. This assumption is reflected in the research literature on chronic pain and coping. Few studies have examined the frequency of nonpain primary stressors in chronic pain patients (Longo, Messier, Nelson, Krishnan, \& Alessi, 1998; Turner, Clancy, \& Vitaliano, 1987). Available studies suggest that the frequency of pain versus nonpain primary stressors varies with severity of pain. For example, in a study of heterogeneous pain patients (chronic low back pain, thoracic outlet syndrome, and reflex sympathetic dystrophy) recruited from a chronic pain clinic, approximately 33\% of the patients described factors other than pain as their primary life stressor; patients who had lower than average pain intensity were more 
likely to report nonpain primary stressors (Longo et al., 1998). Similar results concerning the role of pain intensity were found in a study conducted by Turner et al. (1987), in which a sample of individuals with chronic low back pain was recruited from the community. These individuals had only mild functional impairments and reported significantly lower pain intensity than the subjects in Longo et al.'s study. Individuals recruited from the community were more likely to endorse nonpain stressors, such as work and finance issues, as their primary stressor (57\% cited a nonpain stressor).

There may be significant differences in appraisal and coping associated with pain versus nonpain primary stressors; one study found evidence for such differences. Results of this study indicated that low back pain patients who chose pain as their primary stressor reported significantly greater pain during the previous week, and used less problem-focused coping and more avoidance. In addition, they rated their stressor as less likely to be resolved in four years and more likely to always be a problem (Turner et al., 1987). These results suggest that although pain may be the primary stressor for many patients seen in chronic pain clinics, it is equally important to consider other nonpain stressors that might be compounding their pain problems.

Cognitive Factors in Coping and Adjustment to Chronic Pain In recent years, there has been a growing interest in 
the role of cognitive processes in the experience of chronic pain. Specifically, cognitive factors have been examined as mediators of behavioral and affective responses to pain. Several types of coping strategies and pain-related beliefs, including self-efficacy beliefs, beliefs about general locus of control, beliefs about the controllability of pain, cognitive errors, and outcome expectancies, have been investigated (Jensen, Turner, Romano, \& Karoly, 1991; Keefe, Dunsmore, \& Burnett, 1992). Patient coping strategies and beliefs about their pain have been found to predict treatment outcome and to be associated with important adjustment outcomes including pain severity, physical and social functioning, psychological adjustment, and reliance on pain medication (Brown \& Nicassio, 1987; Brown, Nicassio, \& Wallston, 1989; Jensen, Turner, et al., 1991; Keefe et al., 1992; Turk \& Rudy, 1991).

It is well known that there are individual differences in adjustment to chronic pain. Some individuals with chronic pain adjust and continue to function relatively well while others exhibit a great deal of physical and affective dysfunction in response to their condition and its associated stresses. Studies have increasingly relied on models of stress and coping to explain differences in adjustment and treatment outcomes among chronic pain patients (Jensen, Turner, et al., 1991; Jensen \& Karoly, 1991). As discussed above, according to these models, chronic pain is viewed as a chronic stressor that patients 
must attempt to manage with coping efforts and strategies (Lazarus \& Folkman, 1984). In a review of the literature on coping with chronic pain, numerous studies were identified that demonstrated that coping is a factor that significantly influences adjustment to chronic pain (Jensen, Turner, et al., 1991). Although largely correlational, these studies suggest that when patients are able to successfully negotiate stressors associated with chronic pain, the impact of these stressors on psychological and physical health is reduced. Thus, it is important to identify the particular coping styles or strategies that may be most effective in decreasing stress.

\section{Coping strategies}

Investigators have classified coping strategies according to several different dimensions. For example, active coping strategies (e.g. exercising, using distraction to ignore the pain, etc.) require active management or selfregulation of one's own pain, whereas passive coping strategies (e.g. medication intake, taking to bed, etc.) involve withdrawing or yielding control of pain management to an outside force or agent (Jensen, Turner, et al., 1991). Alternatively, problem-focused coping strategies are those that involve attempting to solve or alleviate a problem by acting on the environment or oneself, whereas emotionfocused coping strategies entail managing the negative emotions associated with a stressor (Lazarus, 1993; Lazarus \& Folkman, 1984). 
One study suggests that when individuals view pain as their primary stressor, they are less likely to use problemfocused strategies. Further, they are more likely to view pain as a persistent problem that will not be resolved, unlike individuals reporting other primary stressors (Turner et al., 1987). This is consistent with several studies that have found that individuals use more problem-focused coping strategies in situations that they view as changeable or controllable. In contrast, emotion-focused coping strategies tend to be used in situations where individuals perceive themselves as being unable to affect the outcome of events (Lazarus, 1993; Paez, Basbabe, Valdoseda, Velasco, \& Iraurgi, 1995). Iazarus cited evidence that when nothing can be done to change a situation, problem-solving efforts can be counterproductive and lead to negative adjustment outcomes when they fail. Thus, emotion-focused coping strategies would be optimal to use in such situations.

Unfortunately, the reliability of the results of studies that have examined emotion-focused coping or compared problem-focused and emotion-focused coping have been limited by significant problems with the majority of extant measures of emotion-focused coping strategies. Stanton, Danoff-Burg, Cameron, and Ellis (1994) examined extant scales which purport to measure emotion-focused coping. These investigators uncovered numerous problems with these measures. First, they found that many scales aggregate conceptually distinct forms of emotion-focused 
coping including any factors that are not problem-focused. For example, emotion-focused coping scales include items that reflect taking responsibility for problems, cognitive avoidance, emotional expression, and self-deprecation. These scales have also included strategies that are inversely correlated such as emotional avoidance and emotional approach. Second, many emotion-focused measures have confounded emotion-focused coping strategies with emotional outcomes particularly emotional distress and psychopathology. Some items such as "feel a lot of emotional distress and find myself expressing those feelings a lot" combine emotional distress and emotional expression. Other items solely reflect distress such as "become very tense" and "have periods of days, weeks, or months, when you couldn't take care of things because you couldn't get going." Finally, a related problem with emotion-focused scales is the dearth of unconfounded items that reflect coping strategies involving emotional approach (i.e. identifying, understanding, and expressing emotions surrounding a stressful experience), despite the fact that there is evidence that indicates there are benefits to emotional processing and expression in coping with stressors. Because of these problems, it is difficult to draw conclusions about the relationships between specific emotion-focused strategies and adjustment or about the relative efficacy of problem-focused and emotion-focused strategies. 
Considerably more is known about active and passive coping strategies and their relationships with adjustment. The Vanderbilt Pain Management Inventory (VPMI) is one measure that was specifically designed to assess active and passive coping strategies. The VPMI has been used frequently with rheumatoid arthritis patients. Within this population, patients who primarily utilized passive coping strategies demonstrated relatively poor affective and physical adjustment as evidenced by higher levels of reported pain severity, depression, and functional disability (Brown \& Nicassio, 1987; Brown et al., 1989). Findings have been slightly less consistent for the use of active coping strategies. In some studies, active coping strategies have been found to be associated with lower levels of pain severity, depression, and functional disability (Brown \& Nicassio, 1987) while other investigators have suggested that the effects of coping strategies may be more context-sensitive (Smith, Wallston, Dwyer, \& Dowdy, 1997).

In a review of the literature on coping with chronic pain, patients who used passive or emotion-focused coping strategies such as catastrophizing, ignoring and reinterpreting strategies, attention diversion, and praying and hoping typically had high levels of physical and psychological disability. In contrast, patients who perceived themselves as having more control or who relied on active or attentional coping were found to function more 
effectively (Keefe et al., 1992). Thus, the use of passive coping strategies typically has been found to be associated with more negative adaptational outcomes than the use of active coping strategies.

Much of the research on coping with chronic pain has been correlational in design. Although these studies are helpeul in identifying factors that are related to adjustment, they are limiting in terms of establishing causal relationships and determining the effectiveness of specific coping strategies. Experimental studies that have examined the efficacy of specific coping strategies have focused predominately on cognitive-behavioral coping techniques. Fernandez and Turk (1989) conducted a metaanalysis to evaluate the efficacy of cognitive coping strategies in the control of pain. Results revealed that cognitive coping strategies were more effective in alleviating pain than either no-treatment or expectancy controls. Numerous cognitive coping strategies (e.g., pleasant imagery, redirection of attention, pain acknowledging, etc.) were found to be highly effective in reducing pain. No significant differences emerged among these strategies in terms the relative efficacy of specific cognitive coping strategies.

In summary, the studies reviewed suggest that cognitive-behavioral coping techniques can be efficacious in controlling chronic pain. Although active coping strategies appear to provide more benefit than passive ones, their 
effects may depend on the type of situation with which one needs to cope. Also, significantly more research is needed to determine the relative benefits of emotion-focused and problem-focused coping strategies when dealing with specific stressors. The relationship between perceptions of controllability of events or stressors and different types of coping strategies is of particular interest since chronic pain is likely to be a problem that many sufferers view as unalterable.

Pain-related Beliefs

Beliefs that chronic pain patients have about their pain experience have been found to influence adaptation to chronic illness. Beliefs that one has about self-efficacy and controllability of pain appear to have a direct effect on mood and to have an indirect effect on adjustment by influencing whether or not effective coping strategies are used. One of the most robust relationships that has been found is the association between self-efficacy beliefs and coping and adjustment.

Beliefs about self-efficacy are judgments that one makes about one's ability to perform a given behavior. Individuals' beliefs about self-efficacy have been found to influence both the initiation and persistence of behaviors (Bandura, 1977). In addition, self-efficacy beliefs have been found to be associated with the use of problem-focused coping strategies. In a study with heterogeneous chronic pain patients, those patients who believed in their ability 
to use problem-focused coping strategies (e.g. aerobic exercise, use of opioid medication, stretching) employed these strategies more often in coping with their pain than those with low self-efficacy (those who did not believe they were capable of using problem-focused coping strategies) (Turk \& Rudy, 1991).

In another study with a heterogeneous chronic pain population, self-efficacy beliefs were found to predict pain tolerance beyond the effects of pain intensity (Dolce, Crocker, Moletteire, \& Doleys, 1986). Further, selfefficacy beliefs were positively associated with posttreatment work status and performance, exercise level, and participation in social activities, and negatively associated with post-treatment medication usage and ratings of observed pain behavior (Dolce et al., 1986; Kores, Murphy, Rosenthal, Elias, \& North, 1990). For rheumatoid arthritis patients, beliefs in one's ability to perform activities of daily living also were associated with several indicators of psychological adjustment, including high levels of self-esteem, positive affect, and low levels of depression (Blalock, DeVellis, \& DeVellis, 1989). In a longitudinal study with arthritis patients, perceived selfefficacy was found to predict adjustment over time and was negatively associated with post-treatment pain, disability, and depression (Lorig, Chastain, Ung, Shoor, \& Holman, 1989).

In a general sense, locus of control refers to beliefs 
that one has about whether the outcomes of events are under one's own control (internal locus of control) or controlled by external factors including others or fate (external locus of control). When put in the context of chronic pain, one's beliefs about locus of control involve the degree to which one sees himself or herself as having control over pain. Studies involving general beliefs about locus of control predominately have found a relationship between internal locus of control and positive adjustment to chronic pain. In a heterogeneous chronic pain population, patients with an internal locus of control had a tendency to use more active coping strategies and to report less distress and depression in response to their pain (Jensen, Turner, et al., 1991). In addition, they report lower levels of pain intensity (Keefe et al., 1992).

Other studies have specifically focused on locus of control in terms of beliefs patients have about the controllability of their pain. Overall, patients who believe that they have more personal control over their pain consistently show better physical and psychological functioning than those who attribute control to external sources. This relationship continues to hold up after controlling for pain severity. Specifically, perceived control over pain has been associated with greater use of active coping strategies, and lower levels of depression and interference in daily activities. Conversely, perceived helplessness has been found to predict depression, passive 
coping, global health ratings, pain severity, psychosocial impairment, and symptom severity for up to two years after initial ratings (Jensen, Turner, et al., 1991).

Several studies have examined the role of cognitive distortions (e.g., personalization, selective abstraction, etc.) and adjustment in chronic pain patients. In a prospective study using rheumatoid arthritis patients, Keefe, Brown, Wallston, and Caldwell (1989) explored the relationship between a specific cognitive distortion catastrophizing - and adjustment. These investigators found that patients who had more catastrophizing thoughts showed greater pain, disability and depression six months later. Cognitive distortions also have been reliably linked with depressive symptomatology and self-reported pain intensity in chronic pain patients (Jensen, Turner, et al., 1991). In fact, for chronic low back pain and rheumatoid arthritis patients, cognitive distortions have been found to account for a significant percentage of variance in pain and disability levels beyond that accounted for by diseaserelated variables such as pain duration and degenerative change on $x$-rays (Flor \& Turk, 1988). In another study with chronic low back pain patients, cognitive distortions predicted disability beyond that accounted for by pain severity, depression, or number of pain treatments (Smith, Follick, Ahern, \& Adams, 1986). Similarly, cognitive distortions accounted for additional variance in depression beyond that accounted for by disease severity and disability 
(Smith, Peck, Milano, \& Ward, 1988).

Finally, a relationship between adjustment to chronic pain and an additional pain belief - the belief that one is disabled by pain - has been supported in the literature (Jensen, Turner, et al., 1991). One study that explored this relationship found that a group of heterogeneous chronic pain patients who endorsed the belief that they were disabled by their pain demonstrated significantly lower levels of activity, less life satisfaction, and higher levels of professional service utilization after controlling for pain severity (Jensen \& Karoly, 1992). Other cognitions that have been examined have not consistently been associated with adjustment to chronic pain. These include such cognitive variables as attributional style and expectancies about outcome (Jensen, Turner, et al., 1991).

The above studies suggest that certain coping responses and beliefs are related to different adjustment outcomes. Specifically, patients who believe they have the ability to cope with their pain and perform their daily activities, who believe their pain is controllable, who have fewer cognitive distortions about their pain, and who believe they are not severely disabled appear to cope more effectively and have more positive adaptation than those who do not. Methodological and Conceptual Problems with the

\section{Literature}

Although there appear to be fairly consistent findings in the research literature concerning the association of 
cognitive factors with coping and adjustment, there are some methodological and conceptual problems that exist. First, there is a considerable amount of conceptual overlap among measures of cognitive constructs. For example, studies that have focused on patients' beliefs about self-efficacy, locus of control, and controllability of pain have treated these beliefs as independent prediction variables. Not only is it unclear whether or not these are independent, but more importantly, control beliefs may be better conceptualized as manifestations of adjustment or outcome. For example, patients' beliefs about being disabled have been found to predict physical functioning and impairment. It is likely that the belief that one is disabled is more a consequence of one's level of functioning or a reflection of one's perception of that outcome than a mechanism that influences one's level of physical functioning.

Similarly, some measures of coping include items that conceptually overlap with other constructs and often are confounded with measures of adjustment. These problems have the end result of producing spuriously high correlations among cognitive predictors and adjustment outcome measures. One particular example of this problem that recently has been cited by several researchers is the use of the catastrophizing subscale of the Coping strategies Questionnaire, as a cognitive coping strategy. Catastrophizing is not in line with current definitions of coping which involve attempts to manage stressors. Some 
pain researchers have suggested that catastrophizing is better conceptualized as a negative emotional reaction or adjustment outcome (Jensen, Turner, et al., 1991). This leads to a significantly high relationship with depression, which is often used as a measure of psychological adjustment. Other coping strategies measuring behavioral responses to pain, such as the Increasing Activity subscale of the Coping Strategies Questionnaire, are better conceptualized as facets of adjustment or outcome and tend to overlap with commonly used outcome measures of functional status and physical disability (Geisser, Robinson, \& Henson, 1994; Jensen, Turner, et al., 1991; Sullivan \& D'Eon, 1990). Another methodological problem with the literature concerns the use of composite measures of coping. Measures such as the Coping Strategies Questionnaire and the Vanderbilt Pain Management Inventory combine numerous distinct coping strategies into broad coping indices, often corresponding to active and passive coping. Such composite indices may obscure the relationships that exist between individual coping strategies and measures of adjustment. Smith et al. (1997) have attempted to address this problem with the creation of a new, multidimensional measure, the Vanderbilt Multidimensional Pain Coping Inventory, which assesses numerous relatively distinct pain coping strategies. This measure appears to have more predictive power and specificity in delineating relationships between coping and adjustment than its predecessor, the vanderbilt 
Pain Management Inventory. Future research studies that employ this instrument are needed to gain additional information about the different relationships between individual coping strategies and various adjustment outcome measures in diverse chronic pain populations.

Finally, the majority of research findings in this area are based on correlational rather than experimental studies. Thus, causal relationships between cognitive factors, coping, and adjustment have not been established.

Emotional Factors in Coping and Adjustment

While there is much research on how cognitive constructs are related to coping and adjustment in chronic pain, relatively little is known about how emotional constructs are related to coping and adjustment. There is some research evidence to suggest that emotional approach coping and certain emotional processing styles are healthy and associated with a variety of favorable adjustment outcomes. First, the relationship between emotional processing variables and stressful life events will be examined. Then, the role of emotional processing in coping with chronic illness will be explored.

Several studies have begun to examine emotional processing variables as mediators of the physiological and psychological effects of traumatic or stressful life experiences. Just as there are differences in the ways in which individuals cognitively process or respond to stress, there also are individual differences in ways in which 
stressful life experiences are emotionally processed. For example, Pennebaker (1989) has found that there are large individual differences in the ways and the degree to which people disclose traumatic experiences. In general, individuals differ in their ability to identify, understand, and express their emotions; their comfort with and willingness to disclose emotionally stressful experiences; and their utilization of coping strategies that rely on emotional processing or expression. Further, it appears that these individual differences in emotional processing can be associated with important health outcomes. Emotional Inhibition and Disclesure

One important dimension of emotional coping that has been examined is the extent to which individuals either inhibit or disclose their thoughts and feelings about emotionally stressful events. Inhibition involves actively or consciously suppressing one's feelings, thoughts, and behaviors, whereas disclosure involves actively confronting one's experiences and confiding in others.

In response to evidence that the experience of traumatic events and major life stressors can negatively impact one's health, Pennebaker (1985) first presented an inhibition model in which he related the psychological processes of inhibition and disclosure to the cognitive, behavioral, and physiological effects of traumatic life experiences. In his model, Pennebaker proposed that behavioral inhibition, in the form of failing to confide in 
others about traumatic experiences, is stressful and requires a considerable amount of effort and physiological work. Pennebaker and colleagues conducted several studies that provided evidence that short-term inhibition results in physiological changes associated with autonomic arousal, particularly increases in skin conductance (Pennebaker, 1985; Pennebaker, Hughes, \& O'Heeron, 1987). Over time, the increased physiological activity associated with long-term inhibition was thought to serve as a cumulative stressor, making one more vulnerable to a variety of psychological and physical problems, particularly stress-related illnesses and disease processes (Pennebaker, 1985; 1989).

As research utilizing Pennebaker's model proliferated, it was found that the autonomic changes associated with disinhibition alone, probably were not the primary mechanism leading to long-term health benefits. Instead, Pennebaker (in press) has found that it is the cognitive change that occurs as a result of the disclosure process, rather than disinhibition alone, that is the critical element. As a result, Pennebaker replaced his inhibition model with a cognitive processing model. Subsequently, he has provided evidence that it is the lack of cognitive processing and assimilation associated with failing to disclose traumatic experiences to others that can have a variety of deleterious effects. These negative effects include increased psychological conflict, obsessive thought processes, physiological activity, and vulnerability to stress-related 
diseases. Conversely, the act of disclosing both one's feelings and one's thoughts about these experiences can serve to prevent or reverse these effects.

A study by Pennebaker and Beall (1986) examined the aspects of the disclosure process than best predicted physical and psychological benefits. Results indicated that there are more benefits associated with pure emotional discharge than with solely providing a factual account of a stressful experience, processing of both the emotional and factual aspects of the event appears to be necessary to bring about long-term improvements.

Research suggests that thoughts and feelings that are not disclosed are less likely to be cognitively and affectively assimilated and worked through, and more likely to be the focus of obsessive thinking or rumination. This type of obsessive or low-level thinking requires more effortful processing. Further, it has been associated with increased physiological activity and also may increase one's risk for psychological and physical problems (Paez et al., 1995; Pennebaker, 1985).

In contrast, actively confronting and disclosing emotionally upsetting and stressful experiences serves to provide one with an avenue for organizing, structuring, and finding meaning in stressful experiences (Pennebaker, 1985; Pennebaker, 1989). When information is cognitively assimilated, there also is no further need to inhibit, which eliminates the physical and psychological stress associated 
with this process.

Over the past two decades, a body of research literature has developed which provides evidence that disclosure of emotionally stressful events has reliable long-term physiological and psychological benefits. Results of surveys and correlational studies indicate that individuals who confided in others about emotionally stressful experiences including childhood traumas, warrelated experiences and the loss of a spouse had better health outcomes, reduced sympathetic activity, and less rumination than those who did not (Pennebaker, 1989; Pennebaker \& Beall, 1986).

In order to further explore the beneficial effects of emotional disclosure, Pennebaker (1989) developed an experimental paradigm in which individuals are randomly assigned to disclosure or control groups. Subjects in the disclosure group directly confront previously inhibited stressful life events by writing or talking about their thoughts and feelings about these events. In contrast, control subjects write or talk about superficial topics. In general, subjects engage in these tasks for 15-20 minutes each day for three to five days. By and large, these studies have found that subjects in the disclosure groups show short-term increases in physiological arousal and negative mood, but later experience considerable benefits with improvements in physical, psychological, and behavioral functioning over time (Pennebaker, 1989; Pennebaker \& Beall, 
1986).

In support of Pennebaker's (1985) model, several beneficial physiological changes were found to accompany disclosure. For example, disclosure subjects have been found to exhibit decreases in sympathetic arousal and/or improved immune functioning (Pennebaker et al., 1987; Pennebaker, Kiecolt-Glaser, \& Glaser, 1988; Petrie, Booth, Pennebaker, Davison, \& Thomas, 1995). In addition, subjects appear to demonstrate decreases in skin conductance that are commensurate with the degree to which they disclose previously inhibited material. Subjects with the lowest skin conductance while disclosing subsequently have fewer physician visits over time (Pennebaker, 1989).

Experimental studies also have found that disclosure has a direct impact on immune functioning. In one study, disclosure subjects were found to exhibit improved immune functioning, decreased autonomic arousal and health center visits, and more positive mood (Pennebaker et al., 1988). In a study using medical students, those who expressed their emotions about stressful events demonstrated several positive immune changes including higher antibody levels against Hepatitis $B$ at 4 and 6 -month follow-up periods (Petrie et al., 1995). Francis and Pennebaker (1992) found that university employees who wrote about upsetting personal experiences had improved liver enzyme function in the two months after writing. Overall, the greatest improvements in immune functioning were found in those subjects who 
expressed a considerable amount of emotion and who wrote about topics they previously had inhibited (Esterling, Antoni, Fletcher, Margulies, \& Schneiderman, 1994; Pennebaker et al., 1988).

Other health benefits also have been found to be related to disclosure. Several studies have found that individuals who disclose their thoughts and feelings about stressful life events have fewer illness visits to health centers for several months following their disclosure (Pennebaker \& Beall, 1986; Pennebaker, Colder, \& Sharp, 1990; Pennebaker \& Francis, 1996; Pennebaker et al., 1988). In a study with undergraduate students, Pennebaker and Beall (1986) found that disclosure subjects demonstrated a decreased number of illnesses, less restricted activity due to illness, and fewer health center visits in the six months following the experiment.

Additional studies using a wide variety of subject populations have found that relative to controls, disclosers experienced other long-term benefits including more positive mood, better academic performance and adjustment, elevated life satisfaction, decreased work absenteeism, and faster reemployment (Francis \& Pennebaker, 1992; Pennebaker et al., 1990; Pennebaker \& Francis, 1996; Pennebaker et al., 1988; Spera, Buhrfeind, \& Pennebaker, 1994).

In summary, the above studies suggest that emotional coping in response to stressful life events by disclosing and cognitively processing the experience can result in 
improved physical and psychological adjustment. In contrast, emotional inhibition and the failure to translate stressful experiences into language can result in a variety of detrimental effects. It is clear that individuals differ in the extent to which they engage in the disclosure process. It is likely that this emotional coping style is related to several other emotion-related constructs that have been studied, such as alexithymia and meta-mood skills.

\section{Alexithymia}

While emotional inhibition and emotional disclosure can be viewed as specific coping styles, alexithymia is believed to be a fairly stable personality disposition. Alexithymia is a multidimensional personality construct which involves the processing of emotional information and which has been linked with a variety of psychological and physical conditions including chronic pain.

Alexithymia has both affective and cognitive elements. Individuals who are alexithymic can be characterized by their difficulty identifying and describing emotions, difficulty distinguishing between emotions and bodily sensations, and externally-oriented cognitive style, in which thought content more often relates to external details of daily life than to emotions, fantasies, or inner experiences (Bagby, Parker, \& Taylor, 1994). It is believed that these emotional processing deficits negatively affect alexithymic individuals' ability to regulate affect in a 
healthy manner. As a result of inadequate affect regulation skills, alexithymics experience a tonic low-level physiological arousal which is experienced as an undifferentiated negative subjective state (Friedlander, Lumley, Farchione, \& Doyal, 1997).

The concept of alexithymia originally arose out of clinical observations of patients with somatic disorders (Taylor, 1984). Although there is no definitive explanation for the link between alexithymia and physical illness, several hypotheses have been examined. One hypothesis suggests that alexithymia is a risk factor which increases one's susceptibility to the development of a variety of medical conditions (Taylor, Bagby, \& Parker, 1991). According to this hypothesis, alexithymia is believed to generate pathogenic effects on physiological mechanisms which lead to tissue damage and other organic dysfunction (Lumley, Stettner, \& Wehmer, 1996; Taylor et al., 1991). Although alexithymic individuals have been found to demonstrate tonic physiological hyperarousal, there is not compelling research evidence to suggest that the effects of this hyperarousal are substantial enough to produce organic disease (Lumley et al., 1996).

Although the possibility exists that alexithymia may serve to exacerbate already existing organic conditions, a second hypothesis is more likely: alexithymia exerts its primary influence on illness behavior (Lumley et al., 1996; Watson \& Pennebaker, 1989). It has been hypothesized that 
the Iimited awareness and cognitive processing of emotions of alexithymic individuals makes them more prone to focus on or amplify the somatic components of emotional arousal (Taylor et al., 1991). There is evidence to suggest that alexithymics demonstrate more somatosensory amplification and discordance between actual physiological arousal and subjective reports of arousal. Further, alexithymia is associated with neuroticism and negative affect states which tend to increase the reporting of somatic symptoms (Lumley et al., 1996). These cognitive processes may explain why alexithymic individuals report the presence of a wide variety of medical conditions and have higher utilization of health care services. Overall, the increased illness behaviors exhibited by alexithymics typically are considered indicators of poor coping and adjustment in patient populations.

Emotional Approach Coping

As noted earlier, some researchers have classified coping into the two general categories of problem-focused and emotion-focused coping. Lazarus and Folkman (1984) defined problem-focused coping as direct efforts to modify the problem causing distress while emotion-focused coping involves regulating affect surrounding a stressful experience. The emotion-focused coping construct consists of many different emotion-focused strategies, some of which involve emotional approach while others involve emotional avoidance (Paez et al., 1995; Stanton et al., 1994). 
Emotional approach strategies involve acknowledging, understanding, and expressing emotion about a stressful event (Stanton et al., 1994).

The literature on emotional disclosure reviewed earlier suggests that processing and expressing emotions have beneficial effects on psychological and physical health. Because the processes involved in emotional disclosure are facets of emotional approach coping, one would expect similar results from studies using instruments that purportedly measure emotion-focused coping; however, this has not been the case. Instead, emotional approach strategies often have been associated with poor adjustment outcomes (stanton et al., 1994).

One important distinction that is necessary to point out is the difference between emotional approach coping and venting of emotions. It appears that these separate and quite different emotion-focused coping strategies often have been lumped together under the broader construct of emotional discharge. Although expressing one's emotions is an important aspect of emotional approach coping, examining and understanding one's emotions are equally important components. It is these latter elements that often are lacking in the pure venting of emotions. It is likely that there are differential effects associated with coping through emotional approach and coping through venting that might be obscured when these strategies are lumped together. Some support for the notion that these strategies have 
different effects comes from Pennebaker's emotional

disclosure research. In examining the aspects of disclosure that influenced long-term health, Pennebaker (1989) found that it is the insight that is achieved by expressing and becoming aware of one's emotions and related thoughts that primarily produces positive health effects rather than venting of emotions alone. Pennebaker and Beall (1986) found that subjects who wrote about both their thoughts and emotions about stressful events evidenced improved health relative to those who expressed only their thoughts or their emotions. In essence, when emotional confrontation occurs, the benefits come both from the expression of the emotions themselves, particularly those that previously have been inhibited, and from the awareness, understanding, cognitive assimilation, and insight that occur as part of that process. In contrast, there are no reliable long-term improvements in physical health that have been associated with venting alone (Pennebaker, 1989). Thus, in considering whether emotional approach coping is adaptive, it is important to ensure that venting and other forms of emotional discharge are not considered to be identical processes.

Stanton and colleagues (1994) presented additional criticisms of the existing measures of emotion-focused coping. As described in detail earlier, their examination of emotion-focused measures revealed that these measures contain very few items that tap important components of 
emotional approach coping such as emotional processing and emotional expression. More often that not, items that were designed to tap these content areas were found to be confounded with negative emotional outcomes such as distress and psychopathology.

In light of the problems with existing measures, these investigators created a scale consisting only of unconfounded emotional approach coping items. When emotional approach coping was assessed using these items, there was evidence to suggest that some individuals benefit from the use of emotional approach coping when dealing with stressors. Interesting sex effects emerged in this study, such that women appeared to benefit from processing and expressing their emotions whereas more negative effects occurred for men who utilized these strategies. In addition, the use of emotional approach strategies had effects on psychological adjustment but did not affect reports of physical symptoms. Overall, these results provide some evidence that at least for women, coping through emotional approach is adaptive rather than dysfunctional as a means of managing stressors. Nonetheless, considerably more information is needed regarding the adaptive value of emotional approach coping for both men and women in diverse situations, given that this type of coping may be adaptive under certain conditions but not others. Similarly, more information is needed concerning the effects of emotional-focused coping on 
health. Although no effects on health status measures were found in the healthy subjects used in the above study, improvements in health might be found in patients who are faced with coping with physical health problems.

\section{Meta-mood skilis}

Both emotional approach coping and emotional disclosure are conceptually related emotional coping styles that require a requisite level of emotional skill or competence to implement. It is clear that individuals differ in their capacity to process and utilize emotional information. A construct that appears to have some utility in explaining these individual differences is meta-mood experience. Metamood experience has been defined as an ongoing reflective process that involves attending to one's moods and emotions, discriminating among them, and regulating them. The skills that are involved in that reflective process are known as meta-mood skills. Meta-mood skills have been conceptualized by some researchers as an operationalization of facets of emotional intelligence, which encompasses mental processes including evaluating and expressing one's own emotions and those of others, regulating one's own emotions and those of others, and using emotions in an adaptive manner to guide one's thinking and actions (Salovey \& Mayer, 1990; Salovey, Mayer, Goldman, Turvey, \& Palfai, 1995).

The Trait Meta-Mood Scale (TMMS) was developed to assess relatively stable differences in the degree to which individuals use meta-mood skills. This scale has been found 
to tap three domains: attention to feelings, clarity of feelings, and mood repair, which involves attempts to terminate negative moods and maintain pleasant ones (Salovey et al., 1995). Early studies using the TMMS have focused on identifying the connections between the three aspects of the meta-mood experience and other emotional processing variables, including ambivalence over emotional expression and alexithymia.

It seems likely that differences in meta-mood skilis would affect one's psychological health and that individuals with greater emotional competencies would demonstrate greater adaptation to problems or stressors that face them. As stated earlier, it is believed that the negative affect and ruminative thoughts that occur in response to stressful Iife events can be attenuated when information about the stressful experience is cognitively assimilated by emotionally processing or disclosing one's experiences. Salovey et al. (1995) hypothesized that the ability to successfully process such information would depend on one's meta-mood skills (attention, clarity, and regulation of emotions). These investigators examined the role of metamood skills in reducing the negative affect and ruminative thoughts associated with a stressful experience. They found that individuals who reported experiencing their emotions clearly (high clarity) rebounded more quickly from both the negative mood and the ruminative thoughts induced by a stressful experience. Thus, clarity in discriminating 
feelings served to buffer the negative impact of a stressful event.

In addition to the effect that meta-mood experiences have on psychological adjustment, there also is some preliminary evidence that suggests that differences in one's meta-mood skills may have health effects. A recent study suggests that both attention to one's moods and mood repair are related to reports of decreased physical symptoms and illnesses during stressful experiences (Salovey et al., 1995). Nonetheless, future research is needed to confirm this relationship and other potential health effects, particularly in medical populations. Relationships among Emotional Processing Variables

A few studies have attempted to connect and consolidate research on individual emotional processes, coping styles, and dispositions. Some recent studies have begun to explore the relationship between alexithymia and different emotional processes such as emotional disclosure.

In a study examining the correlates of alexithymia, Berenbaum and James (1994) found significant correlations between alexithymia and ambivalence over emotional expression and increased discomfort with negative emotional states. In another study, the relationship between alexithymia and emotional disclosure was examined (Paez et al., 1995). It was found that individuals high on alexithymia had more difficulty with a self-disclosure task. Alexithymic subjects reported making more effort to avoid 
disclosure, and felt that they did not disclose enough. In addition, they had more negative affect balance (increased negative affect and decreased positive affect) and physical symptoms, and showed more rumination and cognitive avoidance of the traumatic event. Over time, alexithymics who had been in the disclosure condition demonstrated an improvement in affect balance and a decrease in their avoidance of the traumatic event two months later. Thus, there is some preliminary evidence which suggests that induced disclosure in alexithymics has positive effects on emotions and assimilation of traumatic events. Emotional Processing in Chronically Ill patients While more research studies are beginning to examine the emotional processing of stressful life events in general, there are still very few studies that have looked specifically at the role of emotional coping in patients with chronic health problems such as chronic pain. Nonetheless, there is some evidence to suggest that certain emotional coping styles may be associated with Eavorable adjustment outcomes in these patients, particularly in chronic pain patients.

One study examined the effects of alexithymia and emotional coping styles in patients coping with a chronic illness (Paez et al., 1995). Women who underwent mastectomies for breast cancer were found to have improved adjustment as a result of emotion confrontation and positive cognitive processing of their illness. Emotion 
confrontation (showing, expressing, and coping directly with emotions associated with a problem) was associated with improved quality of life, increased positive affect, and better affect balance. In contrast, alexithymia, repression, avoidance, and affective discharge (venting emotions) were all associated with negative affect.

A few studies have begun to examine the role of emotional disclosure and emotional processing of stressful life events in chronic pain patients. Lumley, Kelley, and Leisen (1997) examined the role of emotional processing variables in the prediction of pain and adjustment in a rheumatoid arthritis population. These investigators found that both the manner in which patients emotionally processed stressful life events and characteristically expressed emotions were more important than the actual experience of life stress in the prediction of rheumatoid arthritis outcomes, including pain, physical functioning, and affective functioning. Specifically, they found that increased expression of negative emotion was associated with greater pain, increased rumination about stressful life events was related to greater physical dysfunction, and increased ambivalence about emotional expression and increased thought frequency about stressful life events were associated with greater affective dysfunction.

In another study with rheumatoid arthritis patients, the effects of encouraging emotional disclosure on health outcomes were examined (Kelley, Lumley, \& Leisen, 1997). 
Patients who emotionally disclosed about stressful Iife events were found to demonstrate less affective disturbance and better physical functioning in their daily activities, three months following the interaction.

An exploratory clinical study was conducted in which chronic pain patients were compared with healthy controls (Dominguez et al., 1995). Biofeedback, hypnosis, autogenic relaxation, and the emotional disclosure paradigm were examined in terms of their ability to reduce active inhibition and its psychophysiological consequences. Compared to healthy controls, chronic pain patients demonstrated less improvement; however, chronic pain patients who disclosed their emotions about stressful events were able to successfully produce significant increases in peripheral temperature from before and after disclosure which is indicative of decreased sympathetic arousal. These increases in skin temperature tended to predict faster clinical improvement. Finally, chronic pain patients in the disclosure condition evidenced a more rapid transition to relaxation than those in the biofeedback, hypnosis, and autogenic relaxation conditions.

Dominguez et al. (1995) also studied the effects of differences in emotional expression among chronic pain patients. Patients who were less competent in expressing and communicating their emotional states demonstrated the highest distress and suffering levels. In addition, they evidenced highly variable physical symptoms. 
Finally, some attention has been given to the role of alexithymia in chronic pain patients. Overall, a higher incidence of alexithymia has been found in chronic pain patients than in the general population (Fernandez, Sriram, Rajkumar, \& Chandrasekar, 1989; Sriram, Chaturvedi, Gopinath, \& Shanmugam, 1987). Further, chronic pain patients were found to be more alexithymic than individuals in other patient populations, including those seeking treatment for nicotine dependence and moderate obesity (Lumley, Asselin, \& Norman, 1997).

A study with rheumatoid arthritis patients found that those patients who were alexithymic coped more poorly with their pain condition than nonalexithymic pain patients (Jordan \& Lumley, 1993). Specifically, alexithymic patients reported more pain and had less functional capacity and perceived pain control. Further, alexithymic pain patients experienced more depression and anxiety, particularly those patients who failed to use active pain coping strategies.

The above studies suggest that emotional processing variables appear to mediate the effects of stress in both healthy and chronically ill individuals. Individual differences in emotional processing appear to be associated with demonstrable differences in both psychological and physical functioning. Overall, positive health benefits and adjustment outcomes appear to be associated with such emotional constructs as emotional approach coping, emotional disclosure, and the meta-mood skills of attention to 
feelings, clarity of feelings, and mood repair. Conversely, alexithymia, emotional inhibition, and rumination appear to have negative effects on health and adjustment. Methodological and Conceptual problems with the literature Relatively less research attention has been devoted to the role of emotional processing variables in coping and adjustment to stressful life events than to the role of cognitive variables. Although more consistent findings are beginning to emerge, particularly in regard to the effects of emotional disclosure, there have been some methodological problems that can be identified, many of which mirror the problems cited with the cognitive literature.

First, the experimental paradigm developed by Pennebaker (1989) has led to a fair number of experimental studies concerning emotional disclosure which allows some causal inferences to be drawn. Nonetheless, research involving other emotional processing variables have been largely correlational. As this research literature further develops, the relationships identified will need to be tested with experimental designs.

Second, as with the literature on the relationship between cognitive constructs and adjustment to chronic pain, confounded measurement also has plagued the emotional coping Iiterature. As stated earlier, items on many emotionfocused coping scales have been contaminated by psychopathology and emotional distress. Thus, until recently, measures of emotional coping efforts largely have 
been confounded with adjustment outcomes. Stanton et al. (1994) have attempted to remedy this problem by constructing an emotional approach coping scale that is comprised of items that are uncontaminated by distress. Future research using this instrument should contribute to a better understanding of the patterns of relationships between emotional approach coping and adjustment. Further, the relative benefits of emotional approach coping and problemfocused coping can be explored in relation to particular stressors.

Another similar problem with this literature is that composite measures of emotional coping have combined distinct coping strategies. For example, scales often have included items that tap both emotional approach (e.g. "Iet my emotions go," etc.) and emotional avoidance (e.g. "tell myself that it is really not happening to me, "etc.). This likely has had the effect of obscuring the relationships that exist between individual emotion-focused coping strategies and adjustment outcomes. Thus, there is a need to measure conceptually distinct coping strategies separately in future studies.

In conclusion, it is only recently that more reliable and valid measures of constructs such as emotional approach coping have been developed. Now that measures that tap these constructs exist, there is an increased need to begin connecting and consolidating emotional constructs. There appears to be a significant amount of overlap among these 
constructs and redundancy in measures which have been developed to assess them. For example, it is likely that there is a considerable amount of shared variance among measures of emotional disclosure, emotional approach coping, and meta-mood skills. Eurther, alexithymia and meta-mood skills may be at opposite ends of the same continuum. A similar statement can be made about alexithymia and emotional approach coping. Both emotional approach coping and meta-mood skills involve attending to and acknowledging emotions, discriminating among and understanding emotions, and expressing and regulating emotions. In direct contrast, alexithymia involves deficits in identifying, describing, and regulating emotions.

In addition, there is a need for further examination of the role of emotional approach coping in dealing with a variety of stressors, including the experience of chronic pain. There are numerous measures that have been specifically developed to assess pain-related attributions and beliefs, and pain-related cognitive and behavioral coping techniques. In contrast, there are no pain-specific measures of coping through emotional approach. Thus, although there is evidence that suggests that certain emotional coping styles are related to favorable adjustment outcomes in chronic pain and other behavioral medicine populations, it is likely that pain-specific measures of cognitive constructs will continue to show more robust relationships with pain-specific adjustment outcomes than 
will the more general measures of emotional constructs.

\section{Summary and Specific Aims}

The experience of stress has been linked to the experience of chronic pain in several ways. Individuals demonstrate differences in their response to stress. For some individuals, the characteristic way in which they respond to psychological stress may play a role in the development, maintenance, and exacerbation of chronic pain conditions. Further, the experience of chronic pain itself becomes a stressor with which one must cope.

The effects of stressors, whether premorbid or as a consequence of chronic pain, are mediated by one's cognitive and emotional processing of them. Some individuals with chronic pain have been found to cope well and have favorable adjustment outcomes, including low pain intensity and healthy physical, psychological, and social functioning, whereas others do not. Thus, it is important to identify coping strategies that are effective in decreasing the negative consequences of stress on chronic pain patients.

Much is known about the cognitive factors that predict positive adjustment outcomes in chronic pain patients, including specific pain beliefs and coping strategies. Considerably less is known about the relationship of emotional coping to adjustment. Historically, emotional coping has been associated with negative adjustment outcomes; however, studies of emotional coping have been plagued with conceptual and methodological problems that 
place these findings in question. These questions now may be better answered with new measures that have emerged that remedy some of the problems with older measures. Recently, some evidence has emerged that certain types of emotional coping strategies and competencies also may be associated with favorable adjustment outcomes including physical and emotional health benefits. Unfortunately, very little research attention has been devoted to exploring the role of emotional factors and their contribution to adjustment in the general population or in a population of chronic pain patients. Further, it is not known whether these emotional variables account for variance in adjustment beyond that accounted for by cognitive variables; that is, whether assessment of emotional variables can add anything to the information provided by cognitive variables. Thus, the primary aim of this research study is to determine whether certain types of emotional coping are associated with favorable adjustment outcomes and to provide a better understanding of the unique contribution that emotional coping makes to predicting individual differences in adjustment for a specific group of chronic pain patients those with myofascial pain syndromes.

The proposed research has the following specific aims:

1. To examine the interrelationships among cognitive constructs and to determine the extent to which there is redundancy in the measurement of these constructs.

2. To assess the relationships between cognitive 
constructs, specifically pain-related beliefs and coping strategies, and adjustment (pain, physical impairment, and affective functioning).

3. To examine the interrelationships among emotional constructs and to determine the extent to which there is redundancy in the measurement of these constructs.

4. To assess emotional correlates of adjustment, including alexithymia, emotional approach coping, and metamood skills.

5. To explore how emotional constructs are related to cognitive constructs and to determine whether they make an unique contribution to understanding individual differences in adjustment in chronic pain patients, beyond that of cognitive constructs.

6. To test whether the emotional constructs can predict adjustment beyond what can be predicted by life stress.

7. To determine whether stress and coping interact in the prediction of adjustment.

8. To explore catastrophizing and disclosure as predictors of adjustment outcomes.

9. To explore differences in the prediction of adjustment using a general and pain-specific measure of emotional coping.

\section{Hypotheses}

1. A considerable amount of redundancy will be found among the emotional predictors and among the cognitive 
predictors. Thus, when the measures of the emotional variables are factor analyzed, a smaller number of factors should be derived from these instruments. Similarly, when the measures of the cognitive variables are factor analyzed, a smaller number of factors will emerge.

2. Cognitive constructs, specifically high selfefficacy, high self-control, and the frequent use of active coping strategies (planful problem-solving, positive reappraisal, distraction, and distancing/denial) will be positively associated with adjustment (less pain, less physical impairment, and better mood) in myofascial pain patients.

3. Emotional constructs, specifically high meta-mood skills, and high emotional approach coping will be positively associated with adjustment; whereas, high alexithymia will be inversely associated with adjustment.

4. The emotional constructs listed above will remain associated with adjustment after controlling for the cognitive constructs.

5. High levels of life stress will be inversely correlated with adjustment; however, measures of both cognitive and emotional constructs will correlate with adjustment beyond the association of life stress. 


\section{Methodology}

\section{Participants}

Participants in this study were 80 adults with chronic myofascial pain, including specific myofascial pain syndromes and fibromyalgia. Patients were recruited from clinic rolls and during outpatient clinic visits to the departments of Anesthesiology (Pain Therapy Clinic), Psychiatry and Behavioral Medicine, and Rheumatology, at PennState Geisinger Health System's Geisinger Medical Center, a large regional medical center in rural Pennsylvania. Patients were eligible for participation if at the time of the study, they were age 18 or older and had an existing chronic myofascial pain disorder of six months or more. Individuals were excluded from the study if they had received a diagnosis of dementia, psychosis, or mental retardation. Prior to participating in this study, all participants signed an informed consent form which was approved by the institutional research review boards at Geisinger Medical Center and Wayne State University (see Appendix A) .

A total of 141 patients were identified as meeting eligibility criteria and were asked to participate in this research study. Eighteen patients declined participation. Forty-two patients accepted the packet of questionnaires but did not return it. Eighty-one patients returned the packet of questionnaires. It was decided that patients would be excluded from the study if they returned a packet with more 
than two incomplete questionnaires. As a result, one patient was dropped from the study due to a significant number of incomplete questionnaires, leaving 80 patients remaining in the study. Subjects for whom only one or two questionnaires were incomplete were retained in the study. No questionnaire was left incomplete by more than three subjects.

Study participants ranged in age from 24 to 86 years (mean $=48.67, \mathrm{SD}=11.82)$. Females comprised $75 \%$ of the sample $(n=60)$ and males comprised $25 \%$ of the sample ( $n=$ 20). Due to the geographic region from which the sample was drawn, minority subjects were underrepresented. One subject described her race as Hispanic. All other subjects were Caucasian. Seventy-three percent of subjects described themselves as married or cohabitating, while $27 \%$ were single, divorced, or widowed. Subjects reported a wide range of educational backgrounds with highest level of education completed ranging from 6 th grade to doctoral degree (mean years of education $=12.65, S D=2.35)$. Only $24 \%$ of subjects were employed. Forty-five percent of subjects were receiving disability payments and $23 \%$ were receiving workman's compensation. Pain duration ranged from 6 months to 35 years $($ mean $=11.38, \mathrm{SD}=7.61$ ). In terms of pain location, $66.3 \%$ of subjects reported both upper and lower extremity pain, 21.3\% reported only upper extremity pain, and $12.5 \%$ reported only lower extremity pain. Demographic information is shown in Table 1. All subjects 
Table 1

Demographic Information

\begin{tabular}{|c|c|c|c|}
\hline & $n$ & Mean (SD) & Range \\
\hline Age & & $48.67 \quad(11.82)$ & $24-86$ \\
\hline Education & & $12.65 \quad(2.35)$ & $6-20$ \\
\hline Pain Duration & & $11.38 \quad(7.61)$ & $0.5-35$ \\
\hline \multicolumn{4}{|l|}{ Sex } \\
\hline Male & $20 \quad(25.08)$ & & \\
\hline Eemale & $60 \quad(75.08)$ & & \\
\hline \multicolumn{4}{|l|}{ Race } \\
\hline Caucasian & $78 \quad(97.5 \div)$ & & \\
\hline Hispanic & $1 \quad(1.35)$ & & \\
\hline \multicolumn{4}{|l|}{$\begin{array}{l}\text { Relationship } \\
\text { status }\end{array}$} \\
\hline $\begin{array}{l}\text { Current } \\
\text { Partner }\end{array}$ & $\begin{array}{ll}58 & (72.5 \varepsilon)\end{array}$ & & \\
\hline $\begin{array}{l}\text { No current } \\
\text { Partner }\end{array}$ & $22 \quad(27.58)$ & & \\
\hline \multicolumn{4}{|l|}{ Pain Location } \\
\hline $\begin{array}{l}\text { Upper Extremity } \\
\text { Pain Only }\end{array}$ & $17 \quad(21.35)$ & & \\
\hline $\begin{array}{l}\text { Lower Extremity } \\
\text { Pain only }\end{array}$ & $10 \quad(12.5 \leqq)$ & & \\
\hline Both & $53 \quad(66.33)$ & & \\
\hline Employed & $19 \quad(23.89)$ & & \\
\hline Disability & $36 \quad(45.08)$ & & \\
\hline Work Compensation & $18 \quad(22.55)$ & & \\
\hline
\end{tabular}

$N=80$; for education, $n=79$, for pain duration, $n=77$, for race, $n=$ 79, for disability, $\mathrm{n}=79$, for workmans compensation, $\mathrm{n}=79$ 
reported having sought treatment for their pain in the past. Currently, $82.5 \%$ of subjects reported receiving therapy for their pain. Eighty-four percent of subjects currently take prescription medication for their pain and $61.3 \%$ currently take over-the-counter medication for their pain. Subjects also reported suffering from a variety of additional health problems. Medical history and pain therapy information are shown in Tables 2 and 3 , respectively.

\section{Precedures}

All participants were identified and recruited from clinic rolls and during outpatient visits to the clinics listed in the Participants section above. Written informed consent was obtained from all patients. Participants were assured that all questionnaires and other data would be coded so that their confidentiality would be protected.

Patients were asked to complete a packet of self-report measures including measures of cognitive predictors, emotional predictors, life stress, and adjustment. Due to the length of time needed to complete questionnaires, approximately 90 minutes, patients were allowed to complete questionnaires either at the medical center or at home and to return them in the stamped, self-addressed envelopes that were provided. General demographic information also was obtained from participants including their sex, race, age, marital status, work status, disability status, income, and level of education. In addition, information about patient's medical history was collected including duration 
Table 2

Medical History Information

\begin{tabular}{|c|c|}
\hline & $\mathrm{n}$ \\
\hline Currently taking OTC pain meds & $49 \quad(61.38)$ \\
\hline Currently taking prescription meds & $67 \quad(83.88)$ \\
\hline Antidepressants & $30 \quad(37.53)$ \\
\hline Anxiolytic/Hypnotics & $8 \quad(10.05)$ \\
\hline Headache Medications & $9 \quad(11.3 \%)$ \\
\hline Muscle Relaxants & $15 \quad(18.8 \%)$ \\
\hline NSAIDS & $29 \quad(36.38)$ \\
\hline Opioids & $40 \quad(50.06)$ \\
\hline Opioid Agonists/Antagonists & $(8.8 \%)$ \\
\hline \multicolumn{2}{|l|}{ Additional Medical Problems } \\
\hline Allergies & $(8.8 ?)$ \\
\hline Arthritis & $16 \quad(20.0 \div)$ \\
\hline Autoimmune Disorders & $(6.3 \div)$ \\
\hline Endocrine Disorders & $11 \quad(13.8 \hat{3})$ \\
\hline GI Disorders & $13 \quad(16.3 \div)$ \\
\hline Hiatal Hernia & $(8.8 \%)$ \\
\hline Hypertension & $15 \quad(18.85)$ \\
\hline Mechanical Pain & $20 \quad(25.05)$ \\
\hline Migraine Headaches & $12 \quad(15.03)$ \\
\hline Neuropathic Pain & $6 \quad(7.5 \xi)$ \\
\hline Psychiatric Disorders & $12 \quad(15.0 \leqq)$ \\
\hline Respiratory Disorders & $6 \quad(7.5 \leqq)$ \\
\hline Sensory Disorders & $(5.08)$ \\
\hline Spinal Disorders & $(6.35)$ \\
\hline Other Medical Problems & $20 \quad(25.0 \leqslant)$ \\
\hline
\end{tabular}

$N=80$ 
Table 3

Pain Therapy Information

\begin{tabular}{|c|c|c|}
\hline & $\begin{array}{l}\text { Currently } \\
\text { receiving } \\
\mathbf{n} \\
\end{array}$ & $\begin{array}{l}\text { Received in } \\
\text { past } \\
\text { n } \\
\end{array}$ \\
\hline Any therapy & $66 \quad(82.55)$ & $(100.0 \%)$ \\
\hline Biofeedback & $(8.8 \hat{5})$ & $(35.05)$ \\
\hline Epidural Injections & $13 \quad(16.38)$ & $(47.58)$ \\
\hline Massage Therapy & $(6.35)$ & $(28.73)$ \\
\hline Medications & $(75.08)$ & $(81.38)$ \\
\hline Occupational Therapy & $(05)$ & $(12.53)$ \\
\hline Physical Therapy & $(10.05)$ & $(72.5 \vdots)$ \\
\hline Psychotherapy & $(30.09)$ & $(52.5 \%)$ \\
\hline Steroid Injections & $(17.5 \%)$ & $(56.35)$ \\
\hline Stress Management & $(10.0 \div)$ & $(37.58)$ \\
\hline Surgery & $(10.0 \div)$ & $(46.38)$ \\
\hline Other Pain Therapy & $(16.35)$ & $(6.38)$ \\
\hline
\end{tabular}

$N=80$ 
of pain, location of pain, prescription and over-the-counter medication usage, current pain treatment, past pain treatment, and other health problems. Demographic and medical history information was recorded on a patient information sheet (see Appendix B).

\section{Measures}

\section{Cognitive constructs}

Participants completed measures of cognitive constructs that assess pain coping strategies, and pain specific beliefs including self-efficacy and perceived selfcontrol.

pain coping. The construct of pain coping was assessed by the Vanderbilt Multidimensional Pain Coping Inventory (VMPCI). Subjects completed a 55-item version of the VMPCI (Smith et al., 1997; C. Smith, personal communication, November 19, 1997) (see Appendix C). The VMPCI is comprised of 14 subscales that correspond to 14 purportedly distinct coping strategies: planful problem-solving (e.g., "try to analyze the problem to understand it better"), positive reappraisal (e.g., "make the best of things; try to learn from experience"), distraction (e.g., "do anything to keep my mind off the pain"), confrontative coping (e.g., "take it out on other people"), distancing/denial (e.g., "go on as if nothing happened"), stoicism (e.g., "keep others from knowing how bad things are"), using religion (e.g., "seek God's help"), self-blame (e.g., "blame myself for what's happening"), self-isolation (e.g., "go off by myself to be 
alone"), wishful thinking (e.g., "hope a miracle will happen"), disengagement (e.g., "realize the situation is hopeless and give up"), acceptance (e.g., "learn to Iive with it"), seeking social support ("e.g., try to get advice from someone about what to do"), and venting (e.g., "get upset and let my emotions out"). For this measure, patients rated the frequency with which they engaged in each behavior as a means of coping with their pain. Items were rated on a 5-point scale ranging from "never do when in pain" to "very frequently do when in pain." Subscale scores were calculated by averaging frequency ratings for items in the subscale; higher subscale scores indicate more frequent use of the coping strategy being assessed.

In previous studies, internal consistency coefficients for the VMPCI subscales ranged from 0.60 to 0.92 and 18 month stability coefficients ranged from 0.32 to 0.76 (C) Smith, personal communication, November 19, 1997). In previous studies, VMPCI subscales have been found to correlate with physical functioning, impairment, life satisfaction, and psychological adjustment. When used to predict both positive and negative psychological adjustment, the VMPCI has been found to demonstrate reliable incremental validity over the original Vanderbilt Pain Management Inventory (VPMI), a two-dimensional measure which assesses the broader coping scales of active and passive coping (Smith et al., 1997).

Various subscales on the VMPCI appear to tap different 
forms of coping (e.g. problem-focused coping, emotionfocused coping, etc.). In this study, the VMPCI was examined primarily as a measure of cognitive coping. Thus, subscales that tap aspects of pain coping through predominately cognitive means and that have been associated reliably with positive adaptational outcomes were examined. These subscales include positive reappraisal, planful problem-solving, distraction, and distancing/denial (Smith et al., 1997). In his sample, Smith reported internal consistency coefficients of 0.75 for positive reappraisal, 0.82 for planful problem-solving, 0.63 for distraction, and 0.63 for distancing/denial (C. Smith, personal communication, November 19, 1997). For the sample in this study, internal consistency coefficients were 0.83 for positive reappraisal, 0.77 for planful problem-solving, 0.74 for distraction, and 0.75 for distancing/denial.

self-efficacy. The construct of self-efficacy was assessed by a pain-specific self-efficacy measure, the Chronic Pain Self-Efficacy Scale (CPSS; Anderson, Dowds, Pelletz, Edwards, \& Peeters-Asdourian, 1995) (see Appendix D). The CPSS is a 22-item questionnaire which measures perceived self-efficacy to cope with the effects of chronic pain. The CPSS contains three subscales: Self-Efficacy for Pain-Management (PSE), Self-Efficacy for Coping with Symptoms (CSE), and Self-Efficacy for Physical Function (FSE). Examples of items on the CPSS include: "How certain are you that you can decrease your pain quite a bit" for the 
PSE, "How certain are you that you can control your fatigue" for the CSE, and "How certain are you that you can perform a daily home exercise program" for the FSE. Items were rated on a 10-point Likert scale ranging from "very uncertain" to "very certain." Subscale scores were calculated by averaging the ratings for items in that subscale, with higher scores indicating greater self-efficacy.

Anderson et al. (1995) reported internal consistency coefficients for the three subscales of 0.87 for the PSE, 0.91 for the CSE, and 0.89 for the FSE. In this study sample, internal consistency coefficients were found to be similar, 0.91 for the PSE, 0.90 for the CSE, and 0.89 for the FSE. The study of Anderson et al. (1995) lends some support to the construct validity of the CPSS. Patients with high scores on CPSS subscales have been found to report less severe pain, less interference in their daily lives due to pain, greater activity levels, and greater perceived life control. Further, patients with high scores on CPSS subscales have been found to be significantly less depressed, hopeless, and somatically preoccupied.

Self-Control, Patients' appraisals regarding perceived self-control were assessed by the Self-Control subscale of the West Haven-Yale Multidimensional Pain Inventory (WHYMPI; Kerns, Turk, \& Rudy, 1985) (see Appendix E). This subscale consists of two items which assess the perceived ability to solve problems ("How much control do you feel that you have over your life") and feelings of personal mastery and 
competence ("How much do you feel that you've been able to deal with your problems"). They were rated on a 7-point scale (ranging from "not at all" to "extremely well"). Ratings of items on this scale were averaged with a higher score indicating greater self-control. The self-Control Scale was completed by only 78 patients. The group mean was used as a score for the two patients with missing scores on this measure. An internal consistency coefficient of 0.79 and a two-week stability coefficient of 0.68 were reported for the Self-Control subscale. In previous studies, this subscale has been found to load negatively on general affective distress and positively on activity level factors tapped by the WHYMPI (Kerns, et al., 1985). The internal consistency coefficient for the sample in this study was 0.89.

Emotional Constructs

Measures of the following emotional constructs were used: alexithymia, emotional approach coping, and meta-mood skills (which are akin to emotional competencies).

Alexithymia, Alexithymia was assessed with the 20-item Toronto Alexithymia Scale (TAS-20; Bagby, Parker, \& Taylor, 1994) (see Appendix F). The 20 items that comprise this self-report measure assess limited capacity to identify feelings and distinguish them from the bodily sensations of emotional arousal, inability to communicate feelings to others, and preference for externally oriented thinking. Examples of TAS-20 items include: "I don't know what's going 
on inside me" and "It is difficult for me to find the right words for my feelings." Items were rated on a 5-point Likert scale (ranging from "strongly disagree" to "strongly agree." Items on the TAS-20 were summed with higher scores indicating greater alexithymia.

Internal consistency for the TAS-20 has been found to be 0.81 (Bagby et al., 1994). Test-retest reliability over a three week period was 0.77 . Support has been found for the construct validity of the TAS-20. In previous studies, the TAS-20 correlated negatively with measures of psychological mindedness, need for cognition, and anger expression and correlated positively with a measures of pain, physical symptoms, neuroticism, and poor attentional control (Bagby et al., 1994; Taylor et al., 1991, Lumley et al., 1996). The TAS-20 also has been found to correlate with external observers' ratings of alexithymia characteristics in a behavioral medicine population (Bagby et al., 1994). In this study sample, the internal consistency coefficient was 0.84 .

Emotional approach coping. Emotional approach coping was measured by a scale created by stanton et al. (1994) (see Appendix G). This scale differs from extant emotionfocused coping scales in that items are unconfounded with distress or psychopathology. This scale is comprised of 18 items that assess coping through emotional processing and emotional expression. For example, "I delve into my feelings to get a thorough understanding of them" and "I 
express the feelings I am having." Items were rated on a 4point scale (ranging from "I don't do this at all" to "I do this a lot." Ratings for items on this scale were summed with higher scores indicating more frequent use of emotional approach coping. Stanton et al. (1994) reported an internal consistency of 0.93 . In the sample for this study, the internal consistency coefficient for the emotional approach coping scale was 0.95 . In a stanton et al.'s study, the Emotional Approach Coping Scale was found to predict decreased depression, and increased life satisfaction, at least for women.

Meta-Mood skills, Meta-mood skills, including mood regulation, clarity, and repair, were measured by the Trait Meta-Mood Scale - 30-item Form (TMMS-30; Salovey et al., 1995) (see Appendix H). The TMMS-30 has been hypothesized to assess aspects of emotional intelligence including differences in participants' awareness of their moods and emotions, ability to discriminate among moods, and capacity for regulating emotions (Salovey et al., 1995). The TMMS-30 is comprised of three subscales: Attention to Feelings (e.g. "I pay a lot of attention to how I feel"), Clarity of Feelings (e.g. "I am rarely confused about how I feel"), and Mood Repair (e.g. "No matter how badly I feel, I try to think about pleasant things"). Items were rated on a 5point Iikert scale (ranging from "strongly agree" to "strongly disagree"). Subscale scores were calculated by averaging ratings for items in that subscale. Higher 
subscale scores indicate greater clarity in discriminating feelings, greater competence in regulating moods, and greater capacity to repair negative moods.

Salovey et al. (1995) reported internal consistency coefficients for the TMMS-30 subscales of $0.86,0.88$, and 0.82 , respectively). For this study sample, internal consistency coefficients were found to be 0.69 for Attention, 0.82 for Clarity, and 0.79 for Mood Repair. The TMMS-30 subscales have been found to correlate with other measures of mood and mood management. For example, Attention to Feelings correlated with private and public self-consciousness. Clarity of Feelings was negatively associated with ambivalence over emotional expression and depression. Mood Repair correlated negatively with depression and positively with optimism and beliefs about negative mood regulation (Salovey, 1995).

\section{other Predictors}

In addition to the cognitive and emotional constructs, several other predictors are of interest in this study including life stress, disclosure, and catastrophizing.

Life stress. Life stress was measured using the Life Events Checklist, a list of 32 stressful life events devised by Kelley et al. (1997) (see Appendix I). For this measure, participants were asked to indicate whether they have experienced each of the potentially stressful events, which include items such as the death or serious illness of others, divorce, abuse, declaring bankruptcy, being fired, 
and experiencing violence. Participants also could write in and rate two other stressors. For each event marked, participants were asked to rate, on a 4-point scale, how stressful or difficult the event is for them currently $(0=$ not at all, 1 = mildly stressful, 2 = moderately stressful, and 3 = extremely stressful). Patients' stress intensity scores were summed for all stressors endorsed to produce a total stress score, indicating greater present life stress. The Life Events Checklist was completed by only 77 patients. The group mean was entered for the three patients with missing scores on this measure. In a previous study, greater scores on the Iife Events Checklist have been found to be positively correlated with high levels of secrecy and ambivalence over emotional expression, and inversely correlated with disclosure of stressful life events (Iumley et al., 1997).

Disclosure, The construct of disclosure was measured by the Disclosure portion of the Life Events Checklist (Kelley et al., 1997) (see Appendix I). For this measure, participants were asked to indicate the number of people with whom they have discussed each of the stressors that they reported experiencing $(0=$ none, $1=$ one person, $2=a$ few people, 3 = many people). Ratings for these questions were averaged to yield a Disclosure score. The Disclosure Scale was completed by only 78 patients. The group mean was entered for the two patients missing scores on this measure. High disclosure scores have been found to be related to 
better rheumatoid arthritis status as indicated by less pain, physical dysfunction, and affective disturbance (Lumley et al., 1997).

Catastrophizing. Catastrophizing was explored as a negative emotional reaction or a form of poor emotional coping. This construct was assessed by the Catastrophizing subscale of the Coping strategies Questionnaire (CSQ; Rosentiel \& Keefe, 1983) (see Appendix J). The Catastrophizing subscale contains six items le.g. "When I feel pain, I feel like I can't go on"). Patients were asked to indicate how often they have used each strategy when they have experienced pain. Each item was rated on a 7-point scale (ranging from "never" to "always"). To compute the Catastrophizing subscale score, ratings on items were averaged, with higher numbers indicating greater catastrophizing. The CSQ was completed by only 79 patients. The group mean was entered for the one patient who was missing a score on this measure. Previous studies have found that the Catastrophizing subscale loads on the helplessness factor of the CSQ (Rosentiel \& Keefe, 1983). This subscale also has been found to be correlated positively with measures of poor emotional adjustment as evidenced by high levels of depression and anxiety. When administered to patients in this sample, the internal consistency coefficient was found to be 0.90 .

Pain-specific emotional coping. Unlike the numerous measures of pain-specific cognitive coping that exist, there 
are no similar measures of pain-specific emotional coping. For this study, a pain-specific version of the Emotional Approach Coping Scale also was administered to patients (see Appendix K). This measure was created in order to compare patient's responses to global versus pain-specific measures of emotional coping. For this measure, instructions for the Emotional Approach Coping Scale were modified such that patients were asked to rate items in terms of how frequently they engaged in each coping behavior in coping with their chronic pain. The Emotional Approach Coping Scale - Pain Version was completed by only 77 patients. The group mean was entered for the three patients with missing scores on this measure.

Adjustment outcomes

Patients completed several health status measures that tap three broad areas of adjustment: pain, physical impairment, and affective functioning.

Pain. Pain quality and pain intensity level were measured by the Pain Rating Index (PRI) of the McGill Pain Questionnaire (MPQ; Melzack, 1975) (see Appendix L). The PRI is comprised of 78 adjectives arranged in 20 rankordered categories according to type of pain and pain intensity. Adjectives on the PRI fall into three different classes: words that describe sensory qualities (temporal, spatial, pressure, thermal, and other sensory properties of the pain experience, e.g., pressing, burning, etc.), affective qualities (tension, fear, and autonomic properties 
of the pain experience, e.g., terrifying), and evaluative qualities (the subjective overall intensity of the total pain experience, e.g., "unbearable"). For this study, the window of time given in the instructions was modified such that patients identified adjectives from 20 categories, which best described their pain experience during the past month. It is standard practice to score only the item with the highest rank value when a subject selects more than one word in a category. The PRI yields sensory, affective, evaluative, miscellaneous, and total pain scores constructed from the total rank sum of endorsed adjectives in each category. For this study, only the sensory and affective pain scores were analyzed. The PRI was completed by only 79 patients. The group mean was entered for the patient who was missing a score on this measure.

Test-retest reliability for the PRI has been shown to be quite good. Mean adjective consistency for adjective choice, over the course of three to seven days, was $70.3 \frac{1}{1}$. Internal consistency coefficients range from 0.91 to 0.95 for each category (Melzack, 1975). The PRI is considered by many to be the standard for pain research. There is considerable evidence for the concurrent and predictive validity of the MPQ as a measure of pain quality and pain intensity. Construct validity also has been supported (Wilkie, Savedra, Holzmer, Tesler, \& Paul, 1990). The PRI has been utilized to assess pain quality and intensity, treatment outcome (i.e., pain intensity or quality change), 
and to predict treatment success (Karoly \& Jensen, 1987; Kerns \& Haythornthwaite, 1988; Turk \& Rudy, 1988).

In addition to the PRI, two 100 millimeter Visual Analogue Scales (VAS) were utilized to assess current levels of both sensory and affective components of participants' pain experience (Price, McGrath, Rafii, \& Buckingham, 1983) (see Appendix M). Endpoints for the scale measuring sensory intensity are "no pain sensation" to "the most intense pain sensation imaginable." Endpoints for the scale measuring affective magnitude are "not at all unpleasant" to "the most unpleasant imaginable." Patients were provided with the initial instructions of Price et al. (1983) to help them to understand the difference between pain sensation intensity (how strong the pain feels) and affective magnitude (how unpleasant or disturbing the pain is). Participants were asked to mark the lines on the VAS at the point which best represents their average pain intensity and affective magnitude during the past month. Scores on the vas are the number of millimeters from the extreme left edge of each scale to the subject's mark on that line. When detailed explanations of the difference between the sensory and affective dimensions of pain are provided, these scales have been found to be valid and reliable measures for both the intensity and the unpleasantness of chronic pain (Price et al., 1983). The VAS are standard dependent variables used to assess change in pain experience. The VAS was completed by only 77 subjects. The group mean was used for the three 
patients missing scores on this measure.

Physical impairment. The Interference subscale of the Pain Experience section of the West Haven-Yale Multidimensional Pain Inventory (WHYMPI) was employed to assess the impact of pain on participants' lives and the extent to which they were able to participate in common daily activities during the past month (Kerns et al., 1985) (see Appendix N). The Interference subscale of the WHYMPI, which contains 9 items, assesses pain-related life interference, including interference with family and marital functioning (e.g., "How much has your pain changed the amount of satisfaction you get from family-related activities"), work and work-related activities (e.g., "How much has your pain changed your ability to work"), and social-recreational activities (e.g., "How much has your pain changed your ability to participate in recreational or other social activities"). Items on the Interference subscale were rated on a 7-point scale. Because some of the items on this subscale, such as items pertaining to work and marriage, did not apply to some patients, an additional response category of "Does not apply" was added to this subscale. The Interference subscale score was computed by averaging ratings of all items that patients rated. Higher scores on this measure are indicative of greater interference due to pain.

Kerns et al. (1985) reported an internal consistency coefficient for the Interference subscale of 0.90 with a 
two-week stability coefficient of 0.86 . In this study sample, the internal consistency coefficient was found to be 0.98. Items on the Interference subscale have been found to load positively on the general affective distress, and pain severity and interference factors tapped by the WHYMPI. In general, support has been found for the construct validity of the WHYMPI scales. Research also suggests that the WHYMPI is sensitive to the prediction of treatment outcome (Kerns, Turk, Holzman, \& Rudy, 1985) and pain severity (Kerns \& Haythornthwaite, 1988).

Affective functioning. Affective functioning was assessed using the Center for Epidemiologic Studies Depression Scale (CES-D), a measure of depression, and the Positive and Negative Affect Schedule - Expanded Form (PANAS-X), a measure of the more general factors of positive affect and negative affect.

Depression was assessed with the CES-D (Radloff, 1977) (see Appendix O). The CES-D is a 20-item scale that assesses depressive symptomatology, including general dysphoric mood, lack of positive mood, and vegetative symptoms. Examples of items include, "I feel hopeful about the future" and "I felt depressed." This scale contains relatively fewer items dealing with somatic symptoms than other measures of depressive symptomatology; thus, it is particularly appropriate for use with medical populations. Using a 4-point scale (ranging from "rarely of none of the time" to "most or all of the time"), patients indicated how 
often they experienced each symptom during the past month. Although the original CES-D used a window of one week, a window of one month was used instead, for consistency with other adjustment measures utilized in this study. The CES-D score was computed by summing ratings on this measure.

Radloff (1977) obtained internal consistency coefficients for the CES-D of 0.85 for the general population and 0.90 for psychiatric patients. stability coefficients for the CES-D were found to be 0.67 at onemonth follow-up and 0.59 at two-month follow-up. For this study sample, the internal consistency coefficient was 0.93 . Substantial support for the construct validity of the CES-D has been found. The CES-D has been found to discriminate well between general population and psychiatric samples and to discriminate moderately well among levels of severity within patient groups. The CES-D has correlated positively with other measures of depression and correlated negatively with measures of positive affect (Radloff, 1977).

The PANAS-X was used to measure the general factors of positive affect (PA) and negative affect (NA) (Watson \& Clark, 1994) (see Appendix P). Participants were asked to rate each of the 60 emotion adjectives on a 5-point scale in terms of how they felt during the past month. PA and NA scores were calculated by averaging item ratings for each scale. The PANAS-X was completed by only 79 patients. The group mean was entered for the one patient with a missing score on this measure. 
The internal consistency reliabilities for the PANAS- $X$, using samples in which "past month" was used as the time frame, have been found to be high. Watson and Clark (1994) reported internal consistency reliabilities 0.89 for PA and NA. The internal consistency coefficient for this study sample was 0.78 for $P A$ and 0.91 for NA. When used as a state affect measure ("past week" instructions), two-month stability coefficients have been found to be 0.43 for PA and 0.41 for NA. When used as a trait affect measure (general instructions), two-month stability coefficients ranged from 0.64 to 0.70 for PA and from 0.59 to 0.71 for NA (Watson and Clark, 1994). There is considerable support for the construct validity of both the $\mathrm{PA}$ and $\mathrm{NA}$ scales of the PANAS- $\mathrm{X}$ as measures of the major dimensions underlying intraindividual mood experience (Watson, 1988; Watson \& Clark, 1994; Watson, Clark, \& Tellegen, 1988). In addition, PANAS- $X$ scales have been found to assess affect states that are similar to those measured by other multi-affect inventories such as the Profile of Mood States (POMS) and to show convergence with peer ratings (Watson \& Clark, 1994). 
Results

\section{Qverview of Results and Analyses}

The following is the approach that was used in analyzing data in this study. First, descriptive statistics and internal consistency coefficients (alpha) were calculated for all measures of cognitive predictors, emotional predictors, life stress, and other predictors. Descriptive statistics then were calculated for outcome variables. Next, the number of predictors to be analyzed was reduced, first by examining intercorrelations among variables and then, through factor analyses. The intercorrelations among the new factors were examined, as were the intercorrelations among the outcomes. Next, the relationships among new factors and the outcomes were examined by conducting zero-order correlations and partial correlations, controlling for possible confounding demographic and medical history variables. Next, hierarchical multiple regressions were conducted in order to examine further the relationship between predictors and outcomes. A hierarchical multiple regression was run in order to determine if the two cognitive predictors were independent. Another series of hierarchical multiple regressions were run to determine whether emotional coping predicted outcomes beyond the effects of sociodemographics and cognitive factors. Then, a series of hierarchical multiple regressions were run to determine whether cognitive factors predicted outcomes beyond the effects of 
sociodemographics and emotional coping. Next, a series of hierarchical multiple regressions were run to determine whether cognitive and emotional factors predicted outcomes beyond the effects of sociodemographics and current life stress. Finally, potential interactions between stress and coping in predicting adjustment outcomes, and exploratory moderator analyses were conducted using hierarchical multiple regression analyses.

\section{Descriptive statistics}

Descriptive statistics including means, standard deviations, ranges, and internal consistencies are presented for predictors in Table 4. Normative data on a few of the predictor variables were available for comparison. Patients in this sample reported a slightly lower level of selfcontrol in regard to their pain (mean $=3.20, \underline{S D}=1.47$ ), when compared to the normative sample for this measure (mean $=3.63, \underline{S D}=1.57 ;$ Kerns et al., 1985). Patients in this sample reported engaging in more catastrophizing (mean = 3.14, $\underline{\mathrm{SD}}=1.70$ ) than patients in the normative sample (mean =2.27; (Rosentiel \& Keefe, 1983). On average, patients in this study reported $6.43(\underline{S D}=4.14)$ stressful events and rated their current stress intensity as mildly/moderately stressful. These results can be compared to a study by Lumley et al. (1997) in which rheumatoid arthritis patients reported an average of $7.39(\underline{S D}=4.85)$ stressful events. In this study, patients reported disclosing stressors to "a few people." Patients were found to disclose their 
Table 4

Descriptive statistics for predictors

\begin{tabular}{|c|c|c|c|}
\hline & Mean & Range & Alpha \\
\hline \multicolumn{4}{|l|}{ Cognitive Predictors } \\
\hline Self-Control Scale & $(1.47)$ & $0-6.00$ & 0.89 \\
\hline \multicolumn{4}{|l|}{$\begin{array}{l}\text { Chronic Pain Self- } \\
\text { Efficacy Scale }\end{array}$} \\
\hline $\begin{array}{l}\text { Self-Efficacy for } \\
\text { Pain Management }\end{array}$ & $48.75 \quad(27.01)$ & $10.00-100.00$ & 0.91 \\
\hline $\begin{array}{l}\text { Self-Efficacy for } \\
\text { Physical Function }\end{array}$ & $39.42 \quad(18.25)$ & $10.00-93.33$ & 0.89 \\
\hline $\begin{array}{l}\text { Self-Efficacy for } \\
\text { Coping with symptoms }\end{array}$ & $45.51 \quad(19.14)$ & $10.00-93.33$ & 0.90 \\
\hline \multicolumn{4}{|l|}{$\begin{array}{l}\text { Vanderbilt Multidimensional } \\
\text { Pain Coping Inventory }\end{array}$} \\
\hline Planful Problem-Solving & $(0.92)$ & $0-4.00$ & 0.77 \\
\hline Positive Reappraisal & $(0.92)$ & $0-4.00$ & 0.83 \\
\hline Distraction & $2.22 \quad(0.92)$ & $0-4.00$ & 0.74 \\
\hline Distancing/Denial & $1.86 \quad(0.90)$ & $0-4.00$ & 0.75 \\
\hline \multicolumn{4}{|l|}{ Emotional Predictors } \\
\hline $\begin{array}{l}\text { Emotional Approach } \\
\text { Coping Scale }\end{array}$ & $48.03 \quad(13.18)$ & $21.00-72.00$ & 0.95 \\
\hline Toronto Alexithymia Scale & $55.26(11.58)$ & $36.00-94.00$ & 0.84 \\
\hline \multicolumn{4}{|l|}{ Trait Meta-Mood Scale } \\
\hline Attention Subscale & $(0.49)$ & $2.62-4.85$ & 0.69 \\
\hline Clarity subscale & $(0.72)$ & $1.09-4.82$ & 0.82 \\
\hline Mood Repair Subscale & $(0.87)$ & $1.33-5.00$ & 0.79 \\
\hline \multicolumn{4}{|l|}{ Other Predictors } \\
\hline Catastrophizing Scale & $(1.70)$ & $0-6.00$ & 0.90 \\
\hline Total Stress Score & $(7.40)$ & $0-47.08$ & \\
\hline Disclosure Score & $(0.76)$ & $0-3.00$ & \\
\hline $\begin{array}{l}\text { Pain-Specific Emotional } \\
\text { Approach Coping Scale }\end{array}$ & $44.71 \quad(14.10)$ & $18.00-72.00$ & 0.96 \\
\hline
\end{tabular}

$\mathrm{N}=80$; for Self-Control Scale, $\mathrm{n}=78$, for Emotional Approach Coping Scale - Pain Version, $n=77$, for Catastrophizing Scale, $n=79$, for Total Stress Scale, $n=77$, for Disclosure Scale, $n=78$. 
stressors to slightly fewer people (mean $=2.01, \underline{S D}=0.76$ ) than a sample of rheumatoid arthritis patients (mean $=2.32$, $\underline{S D}=0.72$; Lumley et al., 1997).

Descriptive statistics for outcomes are presented in Table 5. Results from this study were compared with results from studies with similar samples in the chronic pain Iiterature. It is notable that patients in this study reported very high levels of sensory pain (mean $=23.24$, SD $=8.48$ ) as compared to samples previousiy reported in the literature $($ mean $=17.40$ for musculoskeletal pain patients; Melzack \& Katz, 1992) and (mean $=15.20$ for mixed chronic pain patients; Wilkie et al., 1990). Patients in this study also reported higher than average affective pain (mean = $4.67, \underline{\mathrm{SD}}=3.48$ ) as compared to normative samples previously reported in the literature (mean $=3.70$ for musculoskeletal pain patients; Melzack \& Katz, 1992) and (mean $=3.80$ for mixed chronic pain patients; Wilkie et al., 1990). This suggests that patients in this study have a higher level of organically-based pain than previously studied samples and they appear to be more emotionally distressed by their pain than other chronic pain samples. Patients in this sample reported a similar level of physical impairment as indicated by interference in daily activities (mean $=3.64, \underline{S D}=$ 1.54), when compared to the normative sample for this measure $($ mean $=3.74, \underline{\text { SD }}=1.26$; Kerns et al., 1985). Also, notable is the very high level of depression reported by patients in this study (mean $=28.53, \underline{S D}=13.69$ ). This 
Table 5

Descriptive statistics for Outcomes

\begin{tabular}{|c|c|c|c|}
\hline & Mean & Range & Alpha \\
\hline \multicolumn{4}{|l|}{$\begin{array}{l}\text { McGill Pain Rating } \\
\text { Inventory }\end{array}$} \\
\hline Sensory scale & $23.24 \quad(8.48)$ & $4.00-41.00$ & \\
\hline $\begin{array}{l}\text { Affective } \\
\text { Scale }\end{array}$ & $4.67 \quad(3.48)$ & $0-14.00$ & \\
\hline $\begin{array}{l}\text { Visual Analogue Scale- } \\
\text { Sensory }\end{array}$ & $65.68 \quad(23.00)$ & $12.00-100.00$ & \\
\hline $\begin{array}{l}\text { Visual Analogue Scale- } \\
\text { Affective }\end{array}$ & $66.79 \quad(23.03)$ & $11.00-100.00$ & \\
\hline Interference Scale & $3.64 \quad(1.54)$ & $0-6.00$ & 0.98 \\
\hline $\begin{array}{l}\text { Center for } \\
\text { Epidemiologic studies } \\
\text { - Depression scale }\end{array}$ & $28.53 \quad(13.69)$ & $2.00-55.00$ & 0.93 \\
\hline \multicolumn{4}{|l|}{$\begin{array}{l}\text { Positive and Negative } \\
\text { Affect Schedules - } \\
\text { Expanded Form }\end{array}$} \\
\hline $\begin{array}{l}\text { Positive Affect } \\
\text { Scale }\end{array}$ & $(0.79)$ & $1.00-4.60$ & 0.78 \\
\hline $\begin{array}{l}\text { Negative Affect } \\
\text { Scale }\end{array}$ & $2.51 \quad(0.97)$ & $1.10-5.00$ & 0.91 \\
\hline
\end{tabular}

$N=80$; for McGill Pain Rating Inventory, $\mathrm{n}=79$, for Visual Analogue Scale, $\mathrm{n}=77$, for Positive and Negative Affect Schedules, $\mathrm{n}=79$. 
level of depression is slightly higher than that found in the Center for Epidemiologic Studies of Depression normative psychiatric sample (mean $=24.42, \underline{S D}=13.51 ;$ Radloff, 1977). Patients in this study scored much higher on this measure than samples of rheumatoid arthritis patients (means for three studies $=15.80,14.90$, and $13.80 ;$ Blalock, DeVelis, Brown, \& Wallston, 1989).

\section{Data Reduction}

Earlier, it was hypothesized that there would be a considerable amount of redundancy among measures of cognitive and emotional predictors. Correlational analyses were examined in order to begin to examine the relationships among cognitive predictors and among emotional predictors. Next, factor analyses were conducted to reduce the number of predictors used in further analyses.

Relationships among cognitive Variables

First, zero-order correlations were performed on the cognitive variables in order to determine their relationships with each other. The results of these analyses are shown in Table 6 . Results indicated that the three subscales of the Chronic Pain Self-Efficacy Scale were highly correlated with each other. These correlations ranged from $\underline{r}=.67$ to $\underline{r}=.76$. Thus, patients who had high self-efficacy in one domain, such as for pain management, also had high self-efficacy in other domains, such as for physical function and coping with symptoms.

Results of zero-order correlations also revealed that 
Table 6

Zero-Order Correlations among Cognitive Variables

\begin{tabular}{|c|c|c|c|c|c|c|c|c|}
\hline & 1 & 2 & 3 & 4 & 5 & 6 & 7 & 8 \\
\hline I. PSE & --- & $.74^{\star \star \star}$ & $.67 * \star \star$ & .20 & $.23^{\star}$ & .20 & $.29{ }^{\star \star}$ & $.42^{\star \star \star}$ \\
\hline 2. ESE & & $-\infty$ & $.76^{\star \star \star}$ & $.33^{* \star}$ & $.40 \star \star \star$ & $.31^{\star \star}$ & $.43^{\star \star \star}$ & $.43^{\star \star \star}$ \\
\hline 3. CSE & & & -- & $.45^{\star \star \star}$ & $.53 * \star \star$ & $.39^{\star \star \star}$ & $.57^{\star \star \star}$ & $.62^{\star \star \star}$ \\
\hline 4. PPS & & & & -- & $.74^{\star \star \star \star}$ & 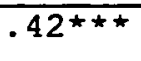 & $.45^{\star \star \star}$ & $.36^{\star \star \star}$ \\
\hline 5. $2 R$ & & & & & -- & $.59^{\star \star \star}$ & $.68^{\star \star \star}$ & $.48 * \star \star$ \\
\hline 6. DIST & & & & & & -- & $.61^{\star \star \star}$ & $.38^{\star \star \star}$ \\
\hline 7. DD & & & & & & & -- & 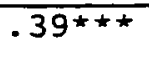 \\
\hline $8 \cdot \operatorname{scs}$ & & & & & & & & $-\infty$ \\
\hline
\end{tabular}

PSE = Self-Efficacy for Pain Management; FSE = Self-Efficacy for Physical Eunction; CSE = Self-Efficacy for Coping with Symptoms; PPS = Planful Rroblem-Solving; $P R=$ Positive Reappraisal; DIST = Distraction; $\mathrm{DD}=$ Distancing/Denial; $\mathrm{SCS}=$ Self-Control Scale.

$\star=\mathrm{p}<.05 ; \star \star=\mathrm{p}<.01 ; \star \star \star=\mathrm{p}<.001$ 
the Vanderbilt Multidimensional Pain Coping Inventory subscales demonstrated significant moderate to large correlations with one another. These correlations ranged from $\underline{r}=.41$ to $\underline{r}=.74$. Thus, patients who reported using cognitive coping strategies, such as positive reappraisal, also reported using additional cognitive coping strategies, such as planful problem-solving, in coping with their chronic pain.

The use of cognitive coping strategies was found to be related to high self-efficacy. Moderate to high correlations were found between Coping with Symptoms and the four cognitive coping skills measured by the Vanderbilt, with correlations ranging from $\underline{r}=.39$ to $\underline{x}=.57$. Moderate correlations were found between Physical Function and the four Vanderbilt subscales with correlations ranging from $\underline{r}=$ .31 to $\underline{\underline{r}}=.43$. Slightly lower correlations were found between Pain Management and the four Vanderbilt subscales with only two of the four correlations reaching significance. Correlations between these scales ranged from $\underline{r}=.20$ to $\underline{r}=.29$. Thus, overall, patients who reported having high self-efficacy also reported utilizing cognitive coping strategies in dealing with their pain.

Finally, the Self-Control Scale correlated significantly with all of the other cognitive predictors. Correlations between the Self-Control scale and the Chronic Pain Self-Efficacy subscales ranged from $\underline{r}=.42$ to $\underline{r}=.62$ and correlations between the Self-Control scale and the 
Vanderbilt scales ranged from $\underline{\underline{ }}=.36$ to $\underline{\underline{r}}=.48$. Thus, patients reporting high control over their pain also tend to have higher self-efficacy and to make more use of cognitive coping strategies in dealing with their pain.

It was decided that the Self-Control scale would not entered into the subsequent factor analysis of cognitive variables because it consists of only two items. In addition, because the Self-Control scale was found to be highly correlated with the three self-Efficacy subscales, it was unlikely that it would contribute a significant amount of additional variance.

Relationships Among Emotional Variables

Zero-order correlations were performed on the emotional variables in order to determine their relationships with each other. The results of these analyses are shown in Table 7. The three subscales of the Trait Meta-Mood Scale demonstrated significant positive correlations with each other with correlations ranging from $\underline{\underline{F}}=.32$ to $\underline{\underline{r}}=.46$, suggesting that the subscales measure related but distinct components of meta-mood experience.

The Toronto Alexithymia scale demonstrated significant negative correlations with the Trait Meta-Mood subscales (Attention, Clarity, and Mood Repair) and the Emotional Approach Coping Scale. The Alexithymia scale was found to correlate $\underline{E}=-.37$ to $\underline{\underline{x}}=-.64$ with the subscales of the Meta-Mood Scale and $\boldsymbol{x}=-.47$ with the Emotional Approach Coping scale. Thus, alexithymic patients reported using 
Table 7

Zero-Order Correlations among Emotional Variables

\begin{tabular}{|l|l|l|l|l|l|}
\hline & 1 & 2 & 3 & 4 & 5 \\
\hline $\begin{array}{l}\text { I. Trait Meta-Mood } \\
\text { Scale - Attention }\end{array}$ & --- & $.36^{\star \star \star}$ & $.32^{\star \star}$ & $-.37^{\star \star \star}$ & $.42^{\star \star \star}$ \\
\hline $\begin{array}{l}\text { 2. } \\
\text { Scalt Meta-Mood }\end{array}$ & & --- & $.46^{\star \star \star}$ & $-.64^{\star \star \star}$ & $.33^{\star \star}$ \\
\hline $\begin{array}{l}\text { Trait Meta-Mood } \\
\text { Scale - Mood Repair }\end{array}$ & & & --- & $-.46^{\star \star \star}$ & .18 \\
\hline $\begin{array}{l}\text { Toronto Alexithymia } \\
\text { Scale }\end{array}$ & & & & --- & $-.47^{\star \star \star}$ \\
\hline $\begin{array}{l}\text { Emotional Approach } \\
\text { Coping Scale }\end{array}$ & & & & & --- \\
\hline
\end{tabular}

$\star=p<.05 ; \star \star=p<.01 ; \star \star \star=p<.001$ 
less emotional approach coping, paying less attention to their moods, having less clarity about their moods, and being less able to repair negative moods than other patients.

The Emotional Approach Coping Scale demonstrated significant correlations with two of the three Meta-Mood subscales, Attention and Clarity $(\underline{r}=.42, \underline{Q}<.001$ and $\underline{\underline{x}}=$ $.33, \mathrm{R}<.001$, respectively). The correlation between the Emotional Approach Coping Scale and the Mood Repair subscale of the Trait Meta-Mood Scale did not reach significance. Thus, patients who reported using emotional approach coping also reported high levels of attention to their moods and clarity about what they are feeling.

Relationships among outcomes

Zero-order correlations were performed on the outcomes to determine their relationships with one another. The results of these analyses are shown in Table 8 . In terms of the pain outcomes, the McGill Sensory and Affective scores were highly related to one another $(\underline{r}=.68, Q<.001)$. Thus, patients who reported high sensory pain also reported high affective pain. The correlation between the sensory Analogue Scale and the Affective Analogue Scale was $\underline{E}=.88$, $Q<.001)$. Patients appeared to be able to distinguish the sensory and affective components of pain more effectively using the McGill Pain Inventory than they were when rating their pain with the two Visual Analogue measures. Patients' ratings on the McGill Sensory and Affective Scales and on 
Table 8

\section{Zero-Order Correlations among Outcomes}

\begin{tabular}{|c|c|c|c|c|c|c|c|c|}
\hline & 1 & 2 & 3 & 4 & 5 & 6 & 7 & 8 \\
\hline $\begin{array}{l}\text { 1. McGill } \\
\text { Sensory } \\
\text { Pain } \\
\text { Scale }\end{array}$ & -- & $.68^{\star \star \star} \star$ & $.41^{\star \star \star}$ & $.41^{\star \star \star}$ & $.41 \star \star \star$ & $.27^{\star}$ & -.20 & $.29^{\star \star}$ \\
\hline $\begin{array}{l}\text { 2. McGill } \\
\text { Affective } \\
\text { Pain Scale }\end{array}$ & & -- & $.25^{*}$ & $.31^{* \star}$ & $.39 * \hbar$ & $.47 *+*$ & $-.35^{\star t}$ & $.47^{\star \star \star \star}$ \\
\hline $\begin{array}{l}\text { 3. Visual } \\
\text { Analogue } \\
\text { Scale - } \\
\text { Sensory }\end{array}$ & & & $-\infty$ & $.88 * \star \star$ & $.60 * \star \star$ & $.30 * t \hbar$ & -.12 & $.28 * t$ \\
\hline $\begin{array}{l}\text { 4. Visual } \\
\text { Analogue } \\
\text { Scale - } \\
\text { Affective }\end{array}$ & & & & -- & $.65 * \star \star$ & $.39 * \star \star$ & $-.24^{\star}$ & $.35^{\star \star}$ \\
\hline $\begin{array}{l}\text { 5. Inter- } \\
\text { ference } \\
\text { Scale }\end{array}$ & & & & & -- & $.45 \star \star \star$ & $.36 * \star \star$ & $.36 \star \star \star$ \\
\hline $\begin{array}{l}\text { 6. Center } \\
\text { for } \\
\text { Epidemio- } \\
\text { logic } \\
\text { Studies - } \\
\text { Depression } \\
\text { Scale }\end{array}$ & & & & & & -- & $-.65 \star \star \star$ & $.78^{\star \star \star}$ \\
\hline $\begin{array}{l}\text { 7. Positive } \\
\text { Affect } \\
\text { scale }\end{array}$ & & & & & & & -- & $-.47 \star \star \star$ \\
\hline $\begin{array}{l}\text { 8. Negative } \\
\text { Affect } \\
\text { Scale }\end{array}$ & & & & & & & & -- \\
\hline
\end{tabular}

$\star=\mathrm{p}<.05 ; \star \star=\mathrm{p}<.01 ; \star \star \star=\mathrm{p}<.001$ 
the Sensory and Affective Analogue Scale were positively correlated. The two sensory measures correlated most highly $(\underline{\underline{x}}=.41, \underline{Q}<.001)$ while the two Affective measures produced a correlation of $\underline{r}=.31, \underline{q}=.005$. These results suggest that patients who report high sensory pain also tend to report high affective pain although this appeared to be more true when patients rated their pain using the VAS than when using the McGill Pain Rating Inventory.

The Interference Scale, a measure of physical impairment, demonstrated a moderate positive relationship with affective and sensory pain. Thus, patients who reported higher pain also reported a greater degree of physical impairment. The Interference scale produced correlations of .41 and .39 with the McGill Sensory and Affective Scales and between .60 and .65 with the Sensory and Affective Analogue Scales.

Measures of affective functioning included the center for Epidemiologic Studies - Depression Scale and the Positive and Negative Affect Schedules - Expanded Version. The Depression Scale was found to correlate highly with the Positive Affect $(\underline{x}=-.65, \underline{Q}<.001)$ and Negative Affect Scales $(\underline{I}=.78, \mathbb{R}<.001)$. Thus, patients who reported higher levels of depression tended to report more negative affect and less positive affect.

The Center for Epidemiologic Studies - Depression Scale correlated positively with the both the McGill sensory ( $\underline{x}=$ $.27, Q=.02)$ and Affective scores $(x=.47, D<.001)$ but 
as expected produced a higher correlation with the Affective score. Similarly, the Depression Scale correlated positively with the sensory $(\underline{\underline{x}}=.30, \underline{2}<.001)$ and Affective $(\underline{x}=.39, \underline{Q}<.001)$ Analogue Scales but had a stronger relationship with the Affective score. Comparable results were found with the Positive and Negative Affect Scales. Higher correlations were found between the positive and Negative Affect Scales and the McGill Affective Scale and the Affective Analogue Scale than between the Positive and Negative Affect Scales and the McGill Sensory Scale and the Sensory Analogue scale. These results indicate that patients with higher levels of sensory and affective pain had higher levels of depression. Results also reveal that reports of affective pain were more highly associated with patients' affective states and depression than their reports of sensory pain.

Finally, depression also was found to be related to physical impairment such that patients who reported more interference in their daily activities due to pain also reported higher levels of depression on the Depression Scale $(\underline{\underline{r}}=.45, \Omega<.001)$. The Positive and Negative Affect Scales demonstrated a similar relationship with the Interference scale. The Interference Scale correlated $\underline{I}=$ $.36, \mathbb{R}=.001$ with the Positive Affect Scale and $\mathscr{E}=.36, \mathfrak{Q}$ $=.001$ with the Negative Affect scale. Thus, patients reporting more interference also reported less positive affect and more negative affect. 
Rather than factor analyzing outcome measures, it was decided instead to retain outcomes as original variables that are widely-used with samples of chronic pain patients. This allows for future comparison with results obtained with other samples of chronic pain patients. In two instances, it was decided that outcome measures simply would be dropped from further analysis in order to eliminate some redundancy in measurement.

First, it was decided that the Sensory and Affective Scales of the McGill Pain Rating Index would be chosen for further analysis, as measures of pain outcomes, over the Sensory and Affective Visual Analogue Scales. Patients' scores on the two visual analogue measures were highly correlated. Thus, it appeared that patients were unable to significantly differentiate sensory and affective pain using this measure. Patients appeared to be able to be able to discriminate sensory and affective pain somewhat better using the MCGill scales. Given this difference and McGill's excellent psychometric properties and longer and more prestigious empirical history, it was decided that the McGill Sensory and Affective Scales would be retained for further analysis instead of the Visual Analogue Scales.

Similarly, it was decided that the Center for Epidemiologic studies - Depression Scale would be used as the measure of affective outcome instead of the Positive and Negative Affect Scales. Both the Bositive and Negative Affect scales correlated quite highly with the Depression 
Scale. In addition, Positive and Negative Affect correlated significantly with each other which suggests that these measures are partially redundant and may not be as independent as has been argued previously. Further, because measures of depression such as the Center for Epidemiologic Studies - Depression Scale have been more standard measures of outcome in chronic pain populations, it was decided to retain the Depression Scale over the Positive and Negative Affect Scales.

\section{Factor Analyses}

As hypothesized, a considerable amount of redundancy was evident both among measures of cognitive and emotional predictors, suggesting the need to limit the number of variables entered into further analyses. It was decided to limit the number of predictors by factor analyzing the set of cognitive predictors and the set of emotional predictors. Factor analyses (principal components with oblique rotation) were performed in order to reduce the number of predictors to a smaller number of factors or dimensions to be used in subsequent analyses.

Factor analysis of cognitive predictors. The variable set for the first factor analysis on the cognitive predictor variables was comprised of the following seven scales: the Positive Reappraisal, Planful Problem-Solving, Distraction, and Distancing/Denial scales of the Vanderbilt Multidimensional Pain Coping Inventory; and the SelfEfficacy for Pain Management, Self-Efficacy for Coping with 
Symptoms, and the Self-Efficacy for Physical Function subscales of the Chronic Pain Self-Efficacy Scale.

Principal components analysis generated two factors with eigenvalues greater than 1.0. The two cognitive factors jointly accounted for 75.4 percent of the total variance. Two factors were extracted and were subjected to an oblique rotation (oblimin). The rotated loadings are presented in Table 9. The first factor can be identified as Cognitive Coping. Positive Reappraisal, Planful ProblemSolving, Distraction, and Distancing/Denial all have strong positive loadings (ranging from .78 to .92) on the factor. The second factor clearly corresponds to Self-Efficacy. Self-Efficacy for Physical Function, Pain Management, and Coping with symptoms all have strong positive loadings on the factor (ranging from .87 to .92) on this dimension.

Based on the results from this factor analysis, two cognitive composite scores were created by combining the variables that had the highest loadings on each factor. Thus, the composite score for "Cognitive Coping" was the average of the four Vanderbilt scales. Descriptive statistics for "Cognitive Coping" are as follows: Mean = $2.09, \underline{\mathrm{SD}}=0.76$, Range $=.06$ to $3.94 \mathrm{~J}$. The composite score for "Self-Efficacy" was the average of the three subscales of the Chronic Pain Self-Efficacy scale. Descriptive statistics for "Self-Efficacy" are as follows: Mean $=43.75$, $\underline{S D}=18.61$, Range $=10.00$ to 91.36$).($ Since cognitive measures for each factor were already measured on the same 
Table 9

Oblimin-Rotated Loadings of the Cognitive Predictors

\begin{tabular}{|l|c|c|}
\hline & Eactor 1 & Eactor 2 \\
& $\begin{array}{l}\text { Cognitive } \\
\text { Coping }\end{array}$ & $\begin{array}{l}\text { Self- } \\
\text { Efficacy }\end{array}$ \\
\hline Planful Problem-Solving & .79 & .31 \\
\hline Positive Reappraisal & .92 & .37 \\
\hline \begin{tabular}{l} 
Distraction \\
\hline $\begin{array}{l}\text { Distancing/Denial } \\
\text { Management }\end{array}$
\end{tabular} & .78 & .28 \\
\hline $\begin{array}{l}\text { Self-Efficay for } \\
\text { Physical Function }\end{array}$ & .25 & .91 \\
\hline $\begin{array}{l}\text { Self-Efficacy for } \\
\text { Coping with Symptoms }\end{array}$ & .59 & .87 \\
\hline $\begin{array}{l}\text { Percent variance } \\
\text { accounted for by factor }\end{array}$ & $55.5 \%$ & 19.93 \\
\hline
\end{tabular}

Loadings printed in bold indicate the factor onto which each variable loaded most highly. 
scale, composites were created by taking an average of the scores).

Factor analysis of emotional predictors. The variable set for the second factor analysis on the emotional predictor variables was comprised of the following five scales: the Toronto Alexithymia Scale, the Emotional Approach Coping Scale, and the Attention to Feelings, Clarity of Feelings, and Mood Repair subscales of the Trait Meta-Mood Scale.

Principal components analysis of the emotional predictors generated one factor with an eigenvalue greater than 1.0. This factor accounted for 52.6 percent of the total variance. All of the emotional predictors loaded strongly on the Emotional Coping factor. Emotional approach coping and the three meta-mood skills of attention, clarity, and mood repair had positive loadings on the Emotional Coping factor (ranging from .65 to .79). Alexithymia had a strong negative loading $(.84)$ on the Emotional Coping factor. Factor loadings are presented in Table 10.

Based on the results from the factor analysis, a composite score was created for the emotion factor, "Emotional Coping." A composite score was created by converting patients' scores on the measures of emotional predictors to standardized scores ( 2 scores) and taking an average of the standard scores. Prior to creating a composite score for "Emotional Coping," scores on the Toronto Alexithymia Scale were reversed because of their 
Table 10

Factor Loadings of the Emotional Predictors

\begin{tabular}{|c|c|}
\hline & $\begin{array}{l}\text { Eactor } 1 \\
\text { Emotional } \\
\text { Coping }\end{array}$ \\
\hline $\begin{array}{l}\text { Trait Meta-Mood Scale - } \\
\text { Attention }\end{array}$ & .66 \\
\hline $\begin{array}{l}\text { Trait Meta-Mood Scale - } \\
\text { Clarity }\end{array}$ & .79 \\
\hline $\begin{array}{l}\text { Trait Meta-Mood Scale - } \\
\text { Mood Repair }\end{array}$ & .66 \\
\hline Toronto Alexithymia scale & -.84 \\
\hline $\begin{array}{l}\text { Emotional Approach Coping } \\
\text { Scale }\end{array}$ & .65 \\
\hline $\begin{array}{l}\text { Percent variance } \\
\text { accounted for by factor }\end{array}$ & $52.6 f$ \\
\hline
\end{tabular}


negative loadings on the emotion factor. Descriptive statistics for "Emotional Coping" are as follows: Mean = 0, $\underline{S D}=0.72$, Range $=-2.26$ to 1.52 .

Exploratory factor analysis of both cognitive and emotional predictors. As an exploratory model, all twelve cognitive and emotional predictors (the Planful ProblemSolving, Positive Reappraisal, Distraction, and

Distancing/Denial scales of the Vanderbilt Multidimensional Pain Coping Inventory; the Pain Management, Physical Function, and coping with Symptoms subscales of the chronic Pain Self-Efficacy Scale; the Toronto Alexithymia Scale; the Attention, Clarity, and Mood Repair subscales of the Trait Meta-Mood Scale; and the Emotional Approach Coping Scale) were entered into a factor analysis in order to determine whether there is support for the conceptual notion of separate dimensions of coping that correspond to cognitive coping and emotional coping. This was considered purely exploratory because of the relatively large number of variables entered into the factor analysis and a less than optimal subject-to-variable ratio.

Principal components analysis of the emotional and cognitive variable sets produced a three factor solution. Three factors accounted for $68.3 \%$ of the total variance. These three factors with eigenvalues greater than 1.0 were extracted and subjected to oblique rotation (oblimin). The rotated factor loadings are presented in Table 11. The first Eactor can again be identified as Cognitive Coping. 
Table 11

Oblimin-Rotated Loadings of the Cognitive and Emotional Predictors

\begin{tabular}{|c|c|c|c|}
\hline & $\begin{array}{l}\text { Eactor } 1 \\
\text { Cognitive } \\
\text { Coping }\end{array}$ & $\begin{array}{l}\text { Factor } 2 \\
\text { Poor Emotional } \\
\text { Coping }\end{array}$ & $\begin{array}{l}\text { Eactor } 3 \\
\text { Self-Efficacy }\end{array}$ \\
\hline $\begin{array}{l}\text { Planful Problem- } \\
\text { Solving }\end{array}$ & .77 & -.45 & .20 \\
\hline $\begin{array}{l}\text { Positive } \\
\text { Reappraisal }\end{array}$ & .92 & -.37 & .32 \\
\hline Distraction & .78 & -.14 & .27 \\
\hline Distancing/Denial & .80 & -.22 & .46 \\
\hline $\begin{array}{l}\text { Self-Efficacy for } \\
\text { Pain Management }\end{array}$ & .21 & -.30 & .88 \\
\hline $\begin{array}{l}\text { Self-Efficacy for } \\
\text { Physical Function }\end{array}$ & .40 & -.24 & .90 \\
\hline $\begin{array}{l}\text { Self-Efficacy for } \\
\text { Coping with } \\
\text { symptoms }\end{array}$ & .57 & -.37 & .86 \\
\hline $\begin{array}{l}\text { Trait Meta-Mood } \\
\text { Scale - Attention }\end{array}$ & .23 & -.75 & .08 \\
\hline $\begin{array}{l}\text { Trait Meta-Mood } \\
\text { scale - Clarity }\end{array}$ & .10 & -.79 & .42 \\
\hline $\begin{array}{l}\text { Trait Meta-Mood } \\
\text { Scale - Mood } \\
\text { Repair }\end{array}$ & .56 & -.52 & .39 \\
\hline $\begin{array}{l}\text { Toronto } \\
\text { Alexithymia scale }\end{array}$ & -.37 & .76 & -.48 \\
\hline $\begin{array}{l}\text { Emotional } \\
\text { Approach Coping } \\
\text { Scale }\end{array}$ & .45 & -.67 & .17 \\
\hline $\begin{array}{l}\text { Percent variance } \\
\text { accounted for by } \\
\text { factor }\end{array}$ & 43.48 & 12.98 & $12.0 \frac{9}{3}$ \\
\hline
\end{tabular}

Loadings printed in bold indicate the factor onto which each variable loaded most highly. 
It is comprised of the four subscales of the vanderbilt Multidimensional Pain Coping Inventory with Positive Reappraisal, Planful Problem-Solving, Distraction, and Distancing/Denial all retaining their strong positive loadings on this dimension. The second factor corresponds to Poor Emotional Coping. Alexithymia had a positive loading on this factor. Emotional approach coping and the meta-mood skills of attention and clarity had negative loadings on this factor. The meta-mood skill of mood repair was approximately equally split between the two factors of Cognitive Coping and Emotional Coping. Finally, a third factor clearly corresponds to the Self-Efficacy factor with the Chronic Pain Self-Efficacy subscales of self-efficacy for physical function, pain management, and coping with symptoms producing strong positive loadings on this factor. Thus, the analysis lends some preliminary support to the notion of maintaining separate cognitive and emotional factors.

\section{Relationships Among Predictors}

The relationships among the new factors (composite scores for Cognitive Coping, Self-Efficacy and Emotional Coping) were examined using zero-order correlations. Results of these analyses are shown in Table 12. Moderate positive zero-order correlations were found among the three factors. A correlation of $I=.49, R<.001$ was found between the Cognitive Coping and Self-Efficacy factors. correlation of $\underline{\underline{I}}=.51, \underline{R}<.001$ was found between the 
Table 12

Zero-Order Correlations among Predictors

\begin{tabular}{|c|c|c|c|c|c|c|c|}
\hline & 1 & 2 & 3 & 4 & 5 & 6 & 7 \\
\hline $\begin{array}{l}\text { 1. Cognitive Coping } \\
\text { Eactor }\end{array}$ & --- & $.49 * \star \star$ & $.51 \star \star \star$ & -.11 & $-.39 \star \star \star$ & .10 & $.58 \star \star \star$ \\
\hline $\begin{array}{l}\text { 2. Self-Efficacy } \\
\text { Eactor }\end{array}$ & & --- & $.47^{\star \star \star}$ & -.19 & $-.60^{\star \star \star \star}$ & $.23^{\star}$ & $.30 * \star$ \\
\hline $\begin{array}{l}\text { 2. Emotional } \\
\text { Coping Factor }\end{array}$ & & & $-\infty$ & -.16 & $-.44 * \star \star$ & .19 & $.61 \star \star \star$ \\
\hline $\begin{array}{l}\text { 3. Total Stress } \\
\text { Score }\end{array}$ & & & & --- & .12 & -.06 & -.04 \\
\hline $\begin{array}{l}\text { 5. Catastrophizing } \\
\text { Scale }\end{array}$ & & & & & --- & -.05 & $-.27 \star$ \\
\hline 6. Disclosure Score & & & & & & -- & .10 \\
\hline $\begin{array}{l}\text { 7. Pain-Specific } \\
\text { Emotional } \\
\text { Approach } \\
\text { Coping Scale } \\
\end{array}$ & & & & & & & --- \\
\hline
\end{tabular}

$\star=p<.05 ; \star \star=p<.01 ; \star \star \star=p<.001$ 
Cognitive Coping and Emotional Coping factors. A correlation of $\underline{I}=.47, Q<.001$ was found between the Emotional Coping and the Self-Efficacy factors. Thus, patients with high levels of self-efficacy for coping with chronic pain also reported utilizing cognitive and emotional coping skills to a greater extent than those with low selfefficacy. Similarly, patients who reported greater use of cognitive coping also have greater self-efficacy and use more emotional coping. The same relationship holds for patients who reported greater use of emotional coping in that they also had greater self-efficacy for coping with chronic pain and used more cognitive coping.

In addition to the cognitive and emotional predictors, other predictors of secondary importance were examined. These included measures of life stress, catastrophizing, disclosure, and a pain-specific version of the Emotional Approach Coping Scale. These predictors were examined separately and not factor-analyzed because it is unclear how these predictors relate to traditional cognitive or emotional predictors of adjustment. In fact, one aim of this study was to better understand the nature of their relationships with coping and adjustment. The pain-specific version of the Emotional Approach Coping Scale was analyzed separately from the other emotional predictors in order to maintain consistency with the other emotional predictor variables which were all global in content and because the pain-specific version of the scale has not had any prior 
psychometric properties determined. Results of analyses with these additional predictors are shown in Table 12. First, the relationships between the total stress score, a sum of current stress intensity scores for all stressors endorsed, and the three cognitive and emotional factors were examined. The total stress score did not correlate significantly with any of the cognitive and emotional factors. Next, the relationship between Catastrophizing and the other predictors was examined. The Catastrophizing Scale was correlated inversely with Cognitive Coping $(\underline{r}=-.39, \underline{\underline{p}}<.001)$, Self-Efficacy $(\underline{r}=-$

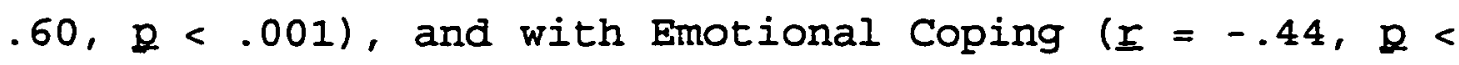
$.001)$. Thus, individuals who engage in more catastrophizing reported using less cognitive coping and less emotional coping. They also reported lower levels of self-efficacy. The Catastrophizing scale was not associated significantly with the Total stress score. Finally, the relationship between Disclosure and the other predictors was examined. The Disclosure Score was correlated positively with the Self-Efficacy factor $(\underline{I}=.23, Q=.04)$. Thus, patients who disclose about stressful life events also have higher levels of self-efficacy. The Disclosure Score was not correlated significantly with any of the other predictors including Cognitive Coping, Emotional Coping, Total Stress, or Catastrophizing. The pain-specific version of the Emotional Approach Scale was correlated positively with Cognitive Coping $(\underline{\Sigma}=.58, R<.001)$, Self-Efficacy $(\underline{x}=.30, \mathrm{R}=$ 
.006), and Emotional Coping $(\underline{\underline{x}}=.61$, $\mathbb{R}<.001)$. (The painspecific version of the Emotional Approach Coping Scale demonstrated a positive correlation of $I=.86,2<.001$ with the original Emotional Approach Coping Scale). The pain-specific version of the Emotional Approach Coping Scale demonstrated an inverse relationship with Catastrophizing ( $\underline{r}$ $=-.27, \mathbb{Q}=.02$ ) but was not correlated with Disclosure or Total stress. Thus, patients who use more emotional approach coping in dealing with their chronic pain also reported using more cognitive and general emotional coping, having more self-efficacy, and catastrophizing less.

Relationships between predictors and Qutcomes

Zero-order correlations also were examined between the cognitive and emotional factors and outcomes. Results of these analyses are shown in Table 13. First, the Cognitive Coping factor was examined in relation to the outcomes. Cognitive Coping correlated negatively with the McGill Affective Scale $(\underline{\underline{I}}=-.27, \underline{\underline{2}}=.02)$ and with the Center for Epidemiologic studies - Depression scale $(\underline{\underline{L}}=-.48, \Omega<$ .001). Cognitive Coping did not correlate significantly with the McGill Sensory Scale or with the Interference scale. Thus, patients who reported frequent use of cognitive coping techniques in dealing with their pain reported less affective pain and less depression.

Next, zero-order correlations were conducted between the Self-Efficacy and the adjustment outcomes. SelfEfficacy correlated inversely with the McGill sensory Scale 
Table 13

Zero-Order Correlations of Predictors with Outcome Variables

\begin{tabular}{|c|c|c|c|c|}
\hline & $\begin{array}{l}\text { McGill } \\
\text { Sensory } \\
\text { Pain }\end{array}$ & $\begin{array}{l}\text { McGill } \\
\text { Affective } \\
\text { Pain }\end{array}$ & $\begin{array}{l}\text { Interference } \\
\text { scale }\end{array}$ & CES-D \\
\hline $\begin{array}{l}\text { Cognitive Coping } \\
\text { Factor }\end{array}$ & -.10 & $-.27 \star$ & -.05 & $-.48 * * *$ \\
\hline $\begin{array}{l}\text { Self-Efficacy } \\
\text { Factor }\end{array}$ & $-.35 * *$ & $-.43 * * *$ & $-.59 * * *$ & $-.65 \star \star \star$ \\
\hline $\begin{array}{l}\text { Emotional Coping } \\
\text { Factor }\end{array}$ & $-.32 * \star$ & $-.48 * * \star$ & $-.26^{\star}$ & $-.66 * t *$ \\
\hline Total Stress Score & .06 & .16 & .04 & $.37 \star \star \star$ \\
\hline $\begin{array}{l}\text { Catastrophizing } \\
\text { Scale }\end{array}$ & $.26^{\star}$ & $.43 * \star \star$ & $.44 \star \star \star$ & $.69 \star \star \star$ \\
\hline Disclosure Score & -.18 & -.18 & $-.23^{\star}$ & -.10 \\
\hline $\begin{array}{l}\text { Pain-Specific } \\
\text { Emotional Approach } \\
\text { Coping Scale }\end{array}$ & $-.26^{\star}$ & $-.38 * \star \star$ & -.18 & $-.40^{* \star \star}$ \\
\hline
\end{tabular}

CES-D = Center for Epidemiological Studies - Depression Scale

$\star=\mathrm{p}<.05 ; \star \star=\mathrm{p}<.01 ; \star \star \star=\mathrm{p}<.001$ 
$(\underline{\underline{I}}=-.35, \mathrm{Q}=.002)$, the McGill Affective Scale, $(\underline{\underline{I}}=$ $.43, \mathfrak{Q}<.001)$, the Interference Scale $(\underline{\underline{x}}=-.59, \underline{\underline{Q}}<.001)$, and the Depression Scale $(\underline{r}=-.65, R<.001)$. Thus, patients with greater self-efficacy for coping with chronic pain reported less sensory and affective pain, less physical impairment, and less depression.

Zero-order correlations were conducted between Emotional coping and the adjustment outcomes. Emotional Coping was correlated inversely with the McGill Sensory Scale $(\underline{\underline{x}}=-.32, \mathfrak{Q}=.004)$, the McGill Affective Scale ( $\underline{\underline{x}}$ $=-.48, \mathbb{R}<.001)$, the Interference Scale $(\underline{\Sigma}=-.26, \mathbb{R}=$ $.02)$, and the Depression Scale $(\underline{\underline{r}}=-.66,2=-.66,2<$ $.001)$. Thus, patients who reported more frequent use of Emotional Coping reported less sensory and affective pain, less physical impairment, and less depression.

Zero-order correlations also were performed between the other predictors and outcomes. Results of these analyses also are presented in Table 13. The total stress score was found to correlate positively with the Depression scale ( $I=$ $.37, \mathrm{R}=.001)$. Thus, patients who reported greater current stress reported greater depression. The Catastrophizing Scale correlated positively with all of the outcome measures. Catastrophizing demonstrated a correlation of $\underline{I}=$ $.26, \mathbb{R}=.02$ with the MCGill sensory scale and a correlation of $\underline{x}=.43, \mathrm{Q}<.001$ with the McGill Affective Scale. Catastrophizing demonstrated a correlation of $\underline{x}=.44,2<$ .001 with the Interference scale and demonstrated a 
correlation of $\underline{\underline{r}}=.69, \underline{\mathrm{Q}}<.001$ with the Depression Scale. These results indicate that patients who demonstrated a high level of catastrophizing had higher levels of sensory and affective pain, physical impairment, and depression than other patients. The Disclosure Score was found to correlate negatively with the Interference Scale $(\underline{\underline{I}}=-.23, \underline{R}=.04)$ but was not significantly related with any of the other outcomes. Thus, patients who disclosed stressful life events had less physical impairment than other patients. Finally, the pain-specific version of the Emotional Approach Coping scale was found to correlate inversely with the McGill Sensory Scale $(\underline{\underline{x}}=-.26, \mathfrak{R}=.02)$, the McGill Affective Scale $(\underline{\underline{r}}=-.38, \underline{Q}=.001)$, and the Depression Scale $(\underline{x}=-.40, \mathbb{R}<.001)$. Thus, patients who used emotional approach coping more frequently in dealing with their pain had less sensory and affective pain and less depression than other patients.

\section{Partial Correlations}

A number of sociodemographic and medical history variables may confound or account for the observed relationships among predictors, or between the cognitive and emotional predictors and the adjustment outcomes. Thus, many studies in health psychology partial out or control for the effects of such variables in their analyses. In this study, age, sex, education, and pain duration were controlled and the relationships tested earlier were reexamined. 
Partial correlations among the predictors (Emotional Coping, Cognitive Coping, Self-Efficacy, Total stress, Catastrophizing, Disclosure, Pain-Specific Emotional Approach Coping) were examined, controlling for age, sex, education, and pain duration. The same significant correlations emerged with the partial correlations as did before with the zero-order correlations.

Partial correlations also were examined among predictors and outcomes. Results of these analyses are shown in Table 14. Results revealed that on the whole the same significant correlations emerged after controlling for demographic variables. Cognitive Coping remained correlated negatively with the McGill Affective Scale and the Depression Scale. Self-Efficacy remained correlated negatively with all of the outcome measures. Emotional Coping remained correlated negatively with the McGill Sensory Scale, the McGill Affective Scale, and the Depression scale but was no longer significantly negatively correlated with the Interference scale after controlling for sociodemographics. The Total Stress Score remained correlated positively with the Depression Scale. The Catastrophizing scale remained correlated positively with all of the outcome measures. The Disclosure Scale no longer correlated significantly with the Interference Scale after controlling for sociodemographics. The pain-specific version of the Emotional Approach Coping Scale remained correlated negatively with the McGill Sensory Scale, the 
Table 14

Partial Correlations of Predictors with Outcomes Controlling for Sex, Age, Education, and Pain Duration

\begin{tabular}{|c|c|c|c|c|}
\hline & $\begin{array}{l}\text { McGill } \\
\text { Sensory } \\
\text { Pain }\end{array}$ & $\begin{array}{l}\text { McGill } \\
\text { Affective } \\
\text { Pain }\end{array}$ & $\begin{array}{l}\text { Interference } \\
\text { Scale }\end{array}$ & CES-D \\
\hline $\begin{array}{l}\text { Cognitive Coping } \\
\text { Factor }\end{array}$ & -.10 & $-.30 * *$ & -.10 & $-.49 * * \star$ \\
\hline Self-Efficacy Eactor & $-.32 * *$ & $-.42^{\star \star \star}$ & $-.57 \star \star \star$ & $-.63 * \star \star$ \\
\hline $\begin{array}{l}\text { Emotional Coping } \\
\text { Factor }\end{array}$ & $-.27 \star$ & $-.43^{\star \star t}$ & -.21 & $-.64 \star \star \star$ \\
\hline Total Stress Score & .04 & .12 & .05 & $.34 * \star$ \\
\hline Catastrophizing Scale & $.23^{\star}$ & $.40^{\star \star *}$ & $.42^{\star \star \star \star}$ & $.68 * \star \star$ \\
\hline Disclosure Score & -.15 & -.14 & -.22 & -.08 \\
\hline $\begin{array}{l}\text { Pain-Specific } \\
\text { Emotional Approach } \\
\text { Coping Scale }\end{array}$ & $-.24 *$ & $-.36 * \star *$ & -.20 & $-.40 * * *$ \\
\hline
\end{tabular}

CES-D = Center for Epidemiological Studies - Depression Scale

$\star=\mathrm{p}<.05 ; \star \star=\mathrm{p}<.01 ; \star \star \star=\mathrm{p}<.001$ 
McGill Affective Scale, and the Depression Scale.

\section{Multiple Regression Analyses}

Hierarchical multiple regression analyses were used to predict each of the adjustment outcomes (sensory pain, affective pain, physical impairment, and affective functioning). For each of the outcomes, hierarchical multiple regressions were run in order to address individual hypotheses. Regression analyses were run only for those predictors and outcomes that demonstrated significant partial correlations above, when controlling for age, sex, education, and pain duration.

First, hierarchical multiple regressions were run in order to determine whether the two cognitive factors were both significant independent predictors of outcomes. For these analyses, sociodemographic variables (sex, age, education, and pain duration) were first controlled, followed by one cognitive factor, and then the other. Results revealed that when sociodemographics and Cognitive Coping were controlled, Self-Efficacy remained a significant predictor of sensory and affective pain, as measured by the MCGill Pain Inventory $(Q=.006$ and $Q=.005$, respectively), physical impairment, as measured by the Interference Scale $(Q<.001)$, and depression, as measured by the Center for Epidemiologic Studies - Depression Scale $(q<.001)$. Results for these analyses are shown in Tables 15-18. Regression analyses then were conducted to determine whether Cognitive coping would continue to predict affective pain 
Table 15

Hierarchical Regression of Sensory Pain on Self-Efficacy Controlling For Cognitive Coping and Sociodemographic Variables

\begin{tabular}{|c|l|l|l|l|}
\hline Variable Entered & Beta & Change $R^{2}$ & $T$ & p-value \\
\hline & & & & \\
\hline Demographic Block & & & & \\
\hline Sex & 0.04 & & 0.33 & .74 \\
\hline Pain Duration & -0.08 & & -0.01 & .99 \\
\hline Education & -0.15 & .037 & -0.59 & .56 \\
\hline Cognitive Coping & -0.10 & .009 & -0.85 & .40 \\
\hline Self-Efficacy & -0.39 & .094 & -2.83 & .006 \\
\hline Full Model: $\quad E(6,73)=1.98, \mathrm{R}^{2}=.14, \mathrm{p}=.08$ \\
\hline
\end{tabular}


Table 16

Hierarchical Regression of Affective Pain on Self-Efficacy, Controlling for Cognitive Coping and Sociodemographic Variables

\begin{tabular}{|c|l|l|l|l|}
\hline Variable Entered & Beta & Change $\mathrm{R}^{2}$ & $\mathrm{~T}$ & p-value \\
\hline Demographic Block & & & & \\
\hline Sex & -0.02 & & -0.17 & .87 \\
\hline Age & -0.13 & & -1.03 & .31 \\
\hline Pain Duration & 0.11 & & 0.86 & .39 \\
\hline Education & -0.39 & .142 & -3.49 & .001 \\
\hline Cognitive Coping & -0.29 & .078 & -2.73 & .008 \\
\hline Self-Efficacy & -0.36 & .081 & -2.91 & .005 \\
\hline Full Model: E $(6,73)=5.25, \mathrm{R}^{2}=.30, \mathrm{p}=.000$ & \\
\hline
\end{tabular}


Table 17

Hierarchical Regression of Physical Impaiment on Self-Efficacy, Controliing for Cognitive Coping and Sociodemographic Variables

\begin{tabular}{|c|l|l|l|l|}
\hline Variable Entered & Beta & Change $\mathrm{R}^{2}$ & $\mathrm{~T}$ & p-value \\
\hline Demographic Block & & & & \\
\hline Sex & -0.07 & & -0.67 & .50 \\
\hline Age & 0.18 & & 1.40 & .17 \\
\hline Pain Duration & -0.19 & & -1.41 & .16 \\
\hline Education & -0.19 & .090 & -1.61 & .11 \\
\hline Cognitive Coping & -0.10 & .010 & -0.90 & .37 \\
\hline Self-Efficacy & -0.73 & .337 & -6.60 & .000 \\
\hline Full Model: & $\mathrm{E}(6,73)=9.42, \mathrm{R}^{2}=.44, \mathrm{p}=.000$ & \\
\hline
\end{tabular}


Table 18

Hierarchical Regression of Depression on Self-Efficacy, Controlling for Cognitive Coping and Sociodemographic Variables

\begin{tabular}{|c|l|l|l|l|}
\hline Variable Entered & Beta & Change $\mathrm{R}^{2}$ & $\mathrm{~T}$ & p-value \\
\hline Demographic Block & & & & \\
\hline Sex & -0.03 & & -0.24 & .81 \\
\hline Age & -0.13 & & -1.02 & .31 \\
\hline Pain Duration & -0.10 & & -0.75 & .45 \\
\hline Education & -0.20 & .088 & -1.78 & .08 \\
\hline Cognitive Coping & -0.49 & .221 & -4.87 & .000 \\
\hline Self-Efficacy & -0.52 & .170 & -4.89 & .000 \\
\hline Full Model: $\quad E(6,73)=11.20, \mathrm{R}^{2}=.48, \mathrm{P}=.000$ & \\
\hline
\end{tabular}


and depression beyond the effects of sociodemographics and Self-Efficacy. Results revealed that Cognitive Coping remained a significant predictor of depression $(Q=.04)$ after controlling for sociodemographics and Self-Efficacy (see Table 19) but no longer was a significant predictor of affective pain beyond the effects of sociodemographics and Self-Efficacy.

Second, hierarchical multiple regressions were run in order to determine whether Emotional Coping would continue to significantly predict sensory pain, affective pain, and depression beyond the effects of sociodemographics and the cognitive factors. For these analyses, sociodemographics were entered and controlled for first, then both cognitive factors were entered together and controlled, and the emotional factor was entered last. In cases in which the emotional factor was not found to predict beyond the effects of sociodemographics and the two cognitive factors together, additional analyses were run in order to determine whether the emotional factor would predict beyond the effects of sociodemographics and either the self-efficacy or cognitive coping factors, when entered individually. The regressions revealed that Emotional Coping remained a significant predictor of both affective pain $(Q=.01)$ and depression ( $Q$ $<.001$ ) beyond sociodemographics and both cognitive factors. Results of these analyses are presented in Tables 20-21. Emotional Coping was found to no longer be related significantly to sensory pain when controlling for both 
Table 19

Hierarchical Regression of Physical Impairmert on Cognitive Coping, Controlling for Self-Efficacy and Sociodemographic Variables

\begin{tabular}{|c|l|l|l|l|}
\hline Variable Entered & Beta & Change $\mathrm{R}^{2}$ & $\mathrm{~T}$ & p-value \\
\hline Demographic Block & & & & \\
\hline Sex & -0.07 & & -0.67 & .50 \\
\hline Age & 0.18 & & 1.40 & .17 \\
\hline Pain Duration & -0.19 & & -1.41 & .16 \\
\hline Education & -0.19 & .090 & -1.61 & .11 \\
\hline Self-Efficacy & -0.58 & .295 & -5.95 & .000 \\
\hline Cognitive Coping & 0.28 & .052 & 2.60 & .01 \\
\hline Full Model: & $\mathrm{E}(6,73)=9.42, \mathrm{R}^{2}=.44, \mathrm{Q}=.000$ & \\
\hline
\end{tabular}


Table 20

Hierarchical Regression of Affective Pain on Emotional Coping, Controlling for Cognitive Eactors and Sociodemographic Variables

\begin{tabular}{|c|l|l|l|l|}
\hline Variable Entered & Beta & Change $\mathrm{R}^{2}$ & $\mathrm{~T}$ & p-value \\
\hline Demographic Block & & & & \\
\hline Age & -0.13 & & -1.03 & .31 \\
\hline Education & -0.39 & & -3.49 & .001 \\
\hline Pain Duration & 0.11 & & 0.86 & .39 \\
\hline Sex & -0.18 & .142 & -0.17 & .87 \\
\hline Cognitive Block & & & & .40 \\
\hline Cognitive Coping & -0.10 & & -0.86 & .005 \\
\hline Self-Efficacy & -0.36 & .159 & -2.91 & .01 \\
\hline Emotional Coping & -0.32 & .058 & -2.56 & \\
\hline Full Model: & E $(7,72)=5.78, \mathrm{R}^{2}=.36, \mathrm{p}=.000$ & \\
\hline
\end{tabular}


Table 21

Hierarchical Regression of Depression on Emotional Coping, Controlling for Cognitive Factors and Sociodemographic Variables

\begin{tabular}{|l|l|l|l|l|}
\hline Variable Entered & Beta & Change $\mathrm{R}^{2}$ & $\mathrm{~T}$ & p-value \\
\hline Demographic Block & & & & \\
\hline Age & -0.13 & & -1.02 & .31 \\
\hline Education & -0.20 & & -1.78 & .08 \\
\hline Pain Duration & -0.10 & & -0.75 & .45 \\
\hline Sex & -0.03 & .088 & -0.24 & .81 \\
\hline Cognitive Block & & & & \\
\hline Cognitive Coping & -0.22 & & -2.08 & .04 \\
\hline Self-Efficacy & -0.52 & .391 & -4.89 & .000 \\
\hline Emotional Coping & -0.46 & .122 & -4.69 & .000 \\
\hline Full Model: & E $(7,72)=15.50, \mathrm{R}^{2}=.60, \mathrm{~g}=.000$ & \\
\hline
\end{tabular}


sociodemographics and both cognitive factors simultaneously. Further analyses revealed that Emotional Coping was a significant predictor of sensory pain $(Q=.02)$ when sociodemographics and Cognitive Coping were controlled, as shown in Table 22. Emotional Coping did not predict sensory pain beyond the effects of both sociodemographics and selfEfficacy $(t(73)=-1.35, \mathrm{D}=.18)$.

Next, hierarchical multiple regressions were run in order to determine whether each of the cognitive factors would continue to predict each of the adjustment outcomes beyond the effects of sociodemographics and the Emotional Coping factor. For these analyses, sociodemographics were entered and controlled for first, then the Emotional Coping factor was entered, and then one of the cognitive factors was entered and controlled before testing the other cognitive factor. Further analyses were conducted in which the Self-Efficacy and the Cognitive Coping factors were entered individually after controlling for sociodemographics and the emotional factor. Results revealed that selfEfficacy remained a significant predictor for all of the adjustment outcomes beyond the effects of sociodemographics, the Emotional Coping factor and the Cognitive Coping factor. Results of these analyses are presented in Tables 23-26. Cognitive Coping was no longer a significant predictor of affective pain or depression when controlling for sociodemographics, Emotional Coping, and Self-Efficacy. Cognitive Coping also was not a significant predictor of 
Table 22

Hierarchical Regression of Sensory Pain on Emotional Coping, Controlling for Cognitive Coping and Sociodemographic Variables

\begin{tabular}{|c|l|l|l|l|}
\hline Variable Entered & Beta & Change $\mathrm{R}^{2}$ & $\mathrm{~T}$ & p-value \\
\hline Demographic Block & & & & \\
\hline Age & -0.00 & & -0.01 & .99 \\
\hline Education & -0.15 & & -1.28 & .20 \\
\hline Pain Duration & -0.08 & & -0.59 & .56 \\
\hline Sex & 0.04 & .037 & 0.33 & .74 \\
\hline Cognitive Coping & -0.10 & .009 & -0.85 & .40 \\
\hline Emotional Coping & -0.33 & .065 & -2.31 & .02 \\
\hline Eull Model: & & & & \\
\hline
\end{tabular}


Table 23

Hierarchical Regression of Sensory Pain on Cognitive Eactors, Controlling for Emotional Coping and Sociodemographic Variables

\begin{tabular}{|l|l|l|l|l|}
\hline Variable Entered & Beta & Change $R^{2}$ & $T$ & p-value \\
\hline Demographic Block & & & & \\
\hline Age & -0.00 & & -0.01 & .99 \\
\hline Education & -0.15 & & -1.28 & .20 \\
\hline Pain Duration & -0.08 & & -0.59 & .56 \\
\hline Sex & 0.04 & .037 & 0.33 & .74 \\
\hline Emotional Coping & -0.28 & .071 & -2.42 & .02 \\
\hline Cognitive Block & & & & .02 \\
\hline Self-Efficacy & -0.33 & & -2.44 & \\
\hline Cognitive Coping & 0.21 & .071 & 1.48 & .14 \\
\hline Full Model: & E $(7,72)=2.24, \mathrm{R}^{2}=.18$, & $\mathrm{R}=.04$ & \\
\hline
\end{tabular}


Table 24

Hierarchical Regression of Affective Pain on Cognitive Factors, Controlling for Emotional Coping and Sociodemographic Variables

\begin{tabular}{|l|l|l|l|l|}
\hline Variable Entered & Beta & Change $R^{2}$ & $T$ & p-value \\
\hline Demographic Block & & & & \\
\hline Age & -0.13 & & -1.03 & .31 \\
\hline Education & -0.39 & & -3.49 & .001 \\
\hline Pain Duration & 0.11 & & 0.86 & .39 \\
\hline Sex & -0.02 & .142 & -0.17 & .87 \\
\hline Emotional Coping & -0.43 & .159 & -4.11 & .000 \\
\hline Cognitive Block & & & & \\
\hline Self-Efficacy & -0.30 & & 0.25 & .80 \\
\hline Cognitive Coping & 0.03 & .058 & & \\
\hline Eull Model: & & & & \\
\hline
\end{tabular}


Table 25

Hierarchical Regression of Physical Impairment on Cognitive Factors, Controlling for Emotional Coping and Sociodemographic Variables

\begin{tabular}{|l|l|l|l|l|}
\hline Variable Entered & Beta & Change $\mathrm{R}^{2}$ & $\mathrm{~T}$ & p-value \\
\hline Demographic Block & & & & \\
\hline Age & 0.18 & & 1.40 & .17 \\
\hline Education & -0.19 & & -1.61 & .11 \\
\hline Pain Duration & -0.19 & & -1.41 & .16 \\
\hline Sex & -0.07 & .090 & -0.67 & .50 \\
\hline Emotional Coping & -0.25 & .042 & -1.89 & .06 \\
\hline Cognitive Block & & & & \\
\hline Self-Efficacy & -0.71 & & -6.30 & .000 \\
\hline Cognitive Coping & 0.32 & .309 & 2.66 & .01 \\
\hline Full Model: & E $(7,72)=8.10, \mathrm{R}^{2}=.44, \mathrm{P}=.000$ & \\
\hline
\end{tabular}


Table 26

Hierarchical Regression of Depression on Cognitive Factors, Controlling for Emotional Coping and Sociodemographic Variables

\begin{tabular}{|l|l|l|l|l|}
\hline Variable Entered & Beta & Change $\mathrm{R}^{2}$ & $\mathrm{~T}$ & p-value \\
\hline Demographic Block & & & & \\
\hline Age & -0.13 & & -1.02 & .31 \\
\hline Education & -0.20 & & -1.78 & .08 \\
\hline Pain Duration & -0.10 & & -0.75 & .45 \\
\hline Sex & -0.03 & .088 & -0.24 & .81 \\
\hline Emotional Coping & -0.66 & .376 & -7.20 & .000 \\
\hline Cognitive Block & & & & \\
\hline Self-Efficacy & -0.43 & & -4.49 & .000 \\
\hline Cognitive Coping & -0.02 & .138 & -0.21 & .83 \\
\hline Full Model: & $\mathrm{E}(7,72)=15.50, \mathrm{R}^{2}=.60, \mathrm{p}=.000$ & \\
\hline
\end{tabular}


affective pain $(t(73)=-0.75, Q=.45)$ or depression ( $t$ $(73)=-1.93, Q=.06)$ even when only controlling for sociodemographics and Emotional Coping.

The Relationship between stress and coping

Earlier zero-order and partial correlations between current life stress and adjustment outcomes revealed that current life stress significantly predicted only depression. Because life stress was not related significantly to outcomes other than depression, it was unlikely that controlling for stress would change significantly the relationships between the coping factors and the other three adjustment outcomes. Hierarchical multiple regressions were conducted to determine whether Self-Efficacy, Cognitive Coping, and Emotional Coping would remain significant predictors of depression beyond the effects of sociodemographics and current life stress. Results revealed that Self-Efficacy $(t(73)=-6.65, q<.001)$, Cognitive Coping $(\underline{t}(73)=-4.97, Q<.001$ ), and Emotional Coping ( $t$ $(73)=-7.20, Q<.001)$ remained significant predictors of depression beyond the effects of sociodemographics and current life stress.

Hierarchical multiple regression analyses were conducted in order to determine if there were significant interaction effects between the total stress score and the three factors (Cognitive Coping, Self-Efficacy, and Emotional Coping) in predicting the four adjustment outcomes. Interaction effects were examined by first 
entering main effects, followed by the interaction term of the total stress score multiplied by one of the factors into the multiple regression equation. No significant

interactions were found between current stress and Cognitive Coping, Self-Efficacy, or Emotional Coping in predicting any of the adjustment outcomes.

\section{Exploratory Moderator Analyses}

Some exploratory analyses were conducted in order to test for moderators of the relationship between coping and adjustment. Sex, age, education, and pain duration were all examined as potential moderators. Each of these four variables was tested in interaction with the three factors (Self-Efficacy, Cognitive Coping, and Emotional Coping) in predicting the four outcome variables. Out of the 48 analyses run, six of these analyses were significant using the traditional significance level of $p<.05$. Because of the exploratory nature of these analyses, it was decided not to alter the significance level to a more conservative value. Therefore, the results of these analyses should be viewed as tentative and interpreted with great caution due to the large number of analyses run and the likelihood of making a Type I error. Given those caveats, the following results emerged.

Sex was found to be a significant moderator of the relationship between Cognitive coping and sensory pain ( $t$ $(76)=-2.39, \mathrm{Q}=.02)$. Results were examined for each gender separately. A moderate negative correlation between 
Cognitive Coping and sensory pain was found for men ( $\underline{x}=$ $.49, \Omega=.03$ ) whereas there was a nonsignificant positive relationship between Cognitive Coping and sensory pain for women $(\underline{\underline{r}}=.07, \underline{Q}=.57)$. Pain duration was found to be a significant moderator of the relationship between Cognitive Coping and sensory pain $(t(76)=2.43, \mathbb{R}=.02)$. Subjects were split into short and long pain duration groups using a median split so that results for each group could be examined separately. A moderate negative relationship was found between Cognitive Coping and sensory pain for patients in the short pain duration group $(\underline{\Sigma}=-.39, \underline{R}=.01)$ whereas a nonsignificant positive relationship was found between Cognitive Coping and sensory pain for patients in the long pain duration group $(I=.17, \Omega=.31)$. Pain duration also was a significant moderator of Cognitive Coping and affective pain $(t(76)=2.24, Q=.03)$. A moderate negative relationship was found between Cognitive coping and affective pain for patients in the short pain duration group $(\underline{I}=-.49, \Omega=.001)$ whereas a nonsignificant negative relationship was found between Cognitive Coping and affective pain for the long pain duration group $(\underline{x}=-.03, \mathbb{R}$ $=.84)$. Education was found to be a significant moderator of the relationship between Emotional Coping and sensory pain $(t(76)=2.30, Q=.02)$. A median split was used to Subjects were split into high and low education groups using a median split so that results for each group could be examined separately. A moderate negative correlation 
between Emotional Coping and sensory pain was found for patients in the high education group $(x=-.34, \mathrm{P}=.005$ ) whereas there was a nonsignificant negative relationship between Emotional Coping and sensory pain for patients in the low education group $(\underline{x}=-.08, \underline{p}=.80)$. Education also was a significant moderator of the relationship between Cognitive Coping and sensory pain $(t(76)=2.07, Q=.04)$. In this case, results of correlations between cognitive Coping and sensory pain were nonsignificant for both groups; however, the slopes of the relationships indicated a trend from a moderate negative relationship between Cognitive Coping and sensory pain for the low education group ( $\underline{r}=-$ $.46, \mathrm{Q}=.13$ ) to a slight negative relationship between Cognitive Coping and sensory pain for the high education group $(\underline{\underline{r}}=-.08, \underline{q}=.51)$. Finally, education also was found to be a significant moderator of Cognitive Coping and physical impairment $(t(76)=2.42,2=.02)$. A moderate negative relationship between Cognitive Coping and physical impairment was found for patients in low education group ( $\underline{r}$ $=-.65, \mathrm{Q}=.02)$ while a nonsignificant positive relationship between Cognitive Coping and physical impairment was found for patients in the high education group $(\underline{I}=.002, \underline{z}=.99)$. 
Discussion

Myofascial pain is one of the primary types of chronic pain seen in general practice and chronic pain clinics in the United States. Psychological stress has been implicated as a factor that may play a role in the development, maintenance, and exacerbation of chronic pain conditions, particularly chronic myofascial pain syndromes. Once a chronic pain syndrome is established, pain becomes an additional stressor with which affected patients must cope. Further, the experience of chronic pain often is associated with a wide variety of negative physical, emotional, social, and economic effects. Thus, chronic pain patients must cope with numerous pain and nonpain stressors.

Research evidence suggests that the way in which one responds to stress, including the stress of pain, may be more important than the experience of stress, or pain, itself. The effects of stressors appear to be mediated by one's cognitive and emotional processing of them. Chronic pain patients demonstrate considerable individual

differences in the ways in which they process or cope with stressors. Some individuals cope well and have favorable adjustment outcomes, including low pain intensity and healthy physical, psychological, and social functioning, whereas others do not. Because of the role that psychological stress plays in the etiology and maintenance of chronic myofascial pain syndromes, it is particularly important to identify different processing styles or coping 
strategies that are effective in decreasing the negative consequences of pain and nonpain stressors on chronic myofascial pain patients. When these patients successfully negotiate stressors, including the stress of pain itself, through their coping efforts, the impact of these stressors on their psychological and physical health will be effectively reduced.

Much is known about the cognitive factors that predict adjustment outcomes in chronic pain patients, including specific pain beliefs and cognitive coping strategies. Considerably less is known about the relationship of emotional coping to adjustment. Historically, emotional coping has been associated with negative adjustment outcomes; however, studies of emotional coping have been plagued with conceptual and methodological problems that put these results in question. Recently, some evidence has emerged that suggests that certain types of emotional approach coping strategies and competencies may be associated with favorable adjustment outcomes. Unfortunately, very little research attention has been devoted to exploring the role of emotional factors and their contribution to adjustment in chronic pain patients.

This study examined the relationships of cognitive and emotional coping to adjustment in chronic myofascial pain patients. In essence, four main issues were at the heart of this study. First, one aim of this study was to provide a better understanding of the nature of cognitive and 
emotional coping. It was of particular interest to determine whether the cognitive and emotional predictors in this study were measuring unique constructs or whether there was a substantial degree of overlap among measures which would suggest that a smaller number of constructs actually are being represented. Two additional major aims of this study were to provide a better understanding of the relationship between cognitive coping and adjustment and between emotional coping and adjustment. It was of particular interest to determine which adjustment outcomes cognitive and emotional coping each would predict and to determine whether both cognitive and emotional coping would be able to predict unique variance in adjustment outcomes beyond the effects of each other. Another aim was to provide a better understanding of the role of life stress in predicting adjustment outcomes. Specifically, it was of interest to determine whether cognitive and emotional coping were more important than stress in predicting adjustment outcomes and to determine whether stress and coping interact in the predicting adjustment outcomes. Aims of secondary importance in this study involved providing a better understanding of catastrophizing and disclosure in the prediction of adjustment outcomes and exploring differences in the use of a general and pain-specific measure of emotional coping.

\section{Redundancy among Measures of Constructs}

The first hypothesis of this study was that there would 
be a considerable amount of redundancy among the measures of emotional constructs such that when factor-analyzed, a smaller number of factors would emerge. This hypothesis was supported. All of the emotional variables in the study loaded on one factor which accounted for $52.6 \%$ of the total variance. Emotional approach coping and the three meta-mood skills of Attention, Clarity, Mood Repair loaded positively on this factor whereas Alexithymia loaded negatively on this factor. This lends support to the notion of there being a more global emotional coping factor.

This finding suggests that although there are many measures of emotional competencies available, there likely are a smaller number of actual constructs being measured. It is likely than when trying to predict adjustment and other outcomes, it may be advantageous to examine more global constructs and to begin to work toward organizing emotional constructs into a larger framework. Such a framework might subsume one or more global constructs and their many submanifestations. One potential framework for emotional coping is emotional intelligence. Emotional intelligence has been proposed as a model that may have considerable heuristic value in organizing different emotion-related constructs or competencies that underlie individual differences in emotional processing (Salovey \& Mayer, 1990).

Although the term "emotional intelligence" is relatively new, its conceptual underpinnings have been 
present in the psychology research literature for a considerable period of time. In essence, emotional intelligence is a integrative framework that encapsulates several conceptually-related mental processes involving emotional information. Some of these mental processes include evaluating one's own emotions and those of others, expressing one's emotions, regulating one's own emotions and those of others, and using emotions in an adaptive manner to guide one's thinking and actions (Salovey \& Mayer, 1990).

Recently, attempts have been made to operationalize aspects of the emotional intelligence construct. Constructs such as alexithymia and emotional expression appear to underlie and measure some components of emotional intelligence. Thus, there is some evidence to suggest that emotion-focused coping, alexithymia, and emotional competencies such as meta-mood skills, can be subsumed under a broader construct, emotional intelligence, which is potentially associated with better adjustment outcomes.

Redundancy also was found among the measures of cognitive constructs. When the seven cognitive variables were factor-analyzed, a smaller number of cognitive factors emerged - Self-Efficacy and Cognitive Coping. The three Chronic pain Self-Efficacy Scale subscales (self-efficacy for pain management, physical function, and coping with symptoms) loaded fairly completely onto the Self-Efficacy factor which accounted for $19.9 \%$ of the total variance, and the four Vanderbilt Multidimensional Pain Coping Inventory 
subscales (planful problem-solving, positive reappraisal, distraction, and distancing/denial) loaded fairly completely onto the Cognitive Coping factor which accounted for $55.5 \%$ of the total variance. Unlike the emotional predictors, which could be reduced to one global factor, two cognitive factors emerged. Although these cognitive factors are separate constructs, they were found to be moderately related to one another $(x=.49)$. This is consistent with the coping literature in that a robust relationship often has been found between self-efficacy beliefs and coping. Individuals' beliefs about self-efficacy have been found to influence both the initiation and persistence of coping behaviors (Bandura, 1977). In chronic pain patients, selfefficacy beliefs have been found to be associated with the use of problem-focused coping strategies (Turk \& Rudy, 1991).

It should be noted that the cognitive variables loaded on factors that were consistent with their original scales, that is, the three subscales of the Chronic Pain SelfEfficacy Scale loaded fairly completely on one factor and the four subscales of the Vanderbilt Multidimensional Pain Coping Inventory loaded fairly completely on the second factor. In part, the resultant two factors could be a function of being from separate scales; that is, similar items and response sets might have led subscales to correlate more highly with each other than with another scale. On the other hand, it also is likely that the two 
factors that emerged accurately represent two somewhat separate constructs, pain-related beliefs and pain coping strategies. The self-Efficacy factor in this study appears to tap pain-related beliefs or attitudes, whereas the Cognitive Coping factor in this study appears to tap painspecific cognitive and behavioral coping strategies. The two factors likely represent a fundamental difference between coping appraisals or beliefs about the consequences of pain and one's ability to deal with it, and specific coping actions or purposeful efforts to manage the negative effects of pain. This finding would be consistent with much of the research Iiterature on chronic pain and adjustment which has treated pain-related beliefs and pain coping strategies as separate but related constructs.

An exploratory factor analysis also was conducted in order to determine how many independent factors would emerge when all of the cognitive and emotional predictors were entered. It was of interest to determine whether separate factors would emerge corresponding to cognitive and emotional coping or whether a single global coping factor would emerge. When all cognitive and emotional predictors were factor-analyzed, a three factor solution emerged. These factors roughly corresponded to Cognitive Coping, Self-Efficacy, and Poor Emotional Coping. Interestingly, the Mood Repair subscale of the Trait Meta-Mood Scale demonstrated a slightly higher positive loading on the Cognitive Coping factor than its negative loading on the 
Poor Emotional Coping factor. An examination of items on the Mood Repair subscale provided some explanation for this finding. Items on the Mood Repair subscale included significantly more cognitive content along with the emotional content than those on the other Trait Meta-Mood subscales of Attention and clarity. For example, samples of items on the Mood Repair subscale included "I try to think good thoughts no matter how badly I feel," "When I become upset, I try to remind myself of all the pleasures in life," and "When I am upset I realize that all the 'good things in life' are illusions." In contrast, items on the Attention and Clarity subscales more purely tapped emotional processes. For example, items included, "I am usually very clear about my feelings," "The best way for me to handle my feelings is to experience them to the fullest," and "I feel at ease about my emotions."

Overall, results of the factor analysis of the cognitive and emotional variables lent some preliminary support to the notion of maintaining separate cognitive and emotional factors. Although it is clear that there is shared variance between cognitive and emotional coping, it also appears that there is something unique to cognitive coping and to emotional coping. As will be discussed, each of the three factors not only predict different domains of functioning but also, predict adjustment beyond the effects of the other factors. 


\section{Cognitive Factors and Adjustment}

The next hypotheses in this study dealt with the prediction of adjustment. Adjustment was conceptualized as low levels of sensory and affective pain, physical impairment, and depression. In this study, it was hypothesized that cognitive constructs would be associated with positive adjustment outcomes. This hypothesis was largely supported. Self-Efficacy in particular was found to be associated with positive adaptation in patients with chronic myofascial pain whereas Cognitive Coping was found to be associated with some measures of positive adaptation but not with others. Results of zero-order correlations between the Self-Efficacy factor and outcome measures suggested that myofascial pain patients who have higher self-efficacy experience less sensory pain, affective pain, interference in daily activities due to their pain condition, and depression. These results remained significant after controlling for sociodemographics. Thus, Self-Efficacy was uniformly positive in that it was associated with better adjustment outcomes across all domains.

The finding that chronic myofascial pain patients with high self-efficacy showed better adjustment, less pain, and were functioning better both physically and emotionally than patients with lower self-efficacy is highly consistent with the existing literature in which self-efficacy has been shown to be a robust and reliable predictor of adjustment 
outcomes. Self-efficacy beliefs consistently have been associated with reports of less depression, pain, and physical impairment. For example, greater beliefs in one's ability to manage pain and other symptoms have been found to be related to lower levels of pain intensity, physical disability, and depression (Jensen et al., 1991). This study lends additional support to the strong relationship between self-efficacy beliefs and adjustment in a previously understudied population of chronic pain patients, chronic myofascial pain patients.

Cognitive Coping, the second cognitive factor that emerged from factor analysis, also was examined in this study. Results of zero-order correlations suggested that chronic myofascial pain patients who engage in cognitive pain coping strategies including planful-problem-solving, positive reappraisal, distraction, and distancing/denial, experience less affective pain and less depression. These results remained significant after controlling for sociodemographics. Thus, Cognitive Coping was related to the two outcomes that are emotionally-linked or manifestations of affect in contrast to the sensory and behavioral outcomes.

The finding that chronic myofascial pain patients who frequently used cognitive coping had better affective functioning and found their pain less emotionally distressing than patients who used cognitive coping strategies less frequently also is highly consistent with 
previous studies in the pain coping and adjustment literature. The use of cognitive coping strategies have been reliably linked with better affective outcomes. Studies using the Vanderbilt Multidimensional Pain Coping Inventory (VMPCI) reveal that greater use of active coping strategies, including planful problem-solving, positive reappraisal, distraction, and distancing/denial has been associated reliably with indices of better affective functioning and psychological adjustment. For example, Brown et al. (1989) found significantly higher levels of depression in patients using passive pain coping strategies than in those using active strategies. Similarly, Smith and Wallston (1996) found that active copers reported lower levels of depression and negative affect and higher levels of positive affect and life satisfaction than passive copers. In another study, Smith et al. (1997) found that the use of active coping strategies was associated with greater positive affect in two samples of chronic pain patients. The use of planful-problem solving and positive reappraisal were associated with less negative affect and the use of planful problem-solving and distancing/denial were associated with less depression. In contrast, differences in pain and physical functioning have not been found consistently between active versus passive copers. Smith and wallston (1996) found highly similar levels of pain and physical impairment in rheumatoid arthritis patients who used active coping strategies and those who 
used passive strategies. Similarly, Smith et al. (1997) found that the use of the active pain coping strategies generally were not associated with significantly lower levels of pain or physical impairment in two samples of chronic pain subjects. In summary, the results of this study are consistent with the past literature that suggests that the use of active cognitive pain coping strategies is associated with better psychological functioning but is not associated consistently with pain or physical impairment.

It is of interest that in this study, the use of active cognitive pain coping strategies was associated with decreased affective pain. Previous studies in the pain coping literature that have examined differences in patients who use active versus passive strategies have not differentiated between the sensory and affective components of pain. An important finding of this study is that when the sensory and affective components of pain are differentiated, the relationship of Cognitive coping is clarified. Cognitive Coping does not predict the sensory component of pain but does predict the affective component of pain. This underscores the importance of measuring the two aspects of pain as separate dimensions.

Two major conclusions can be drawn from the results of analyses dealing with cognitive coping and adjustment. First, it is clear that Self-Efficacy and Cognitive Coping are unique factors that are able to make unique predictions about different domains of adjustment. Second, Self- 
Efficacy was found to be a more robust predictor of adjustment outcomes than Cognitive Coping. This finding may have emerged for several reasons. First, Self-Efficacy may be more central to adaptive functioning because of its generality unlike Cognitive Coping, which is more limited, narrow, and specific. Second, it is possible that selfEfficacy is confounded with adjustment which would make correlations between these constructs spuriously high. It is possible that patients make appraisals based on their current level of functioning and that self-efficacy beliefs are simply manifestations of their current level of physical and psychological adjustment. For example, a patient with little pain may conclude, that he or she is efficacious in managing his or her pain. This would suggest that selfefficacy does not influence one's adjustment but that adjustment influences one's self-efficacy beliefs. Third, an examination of the content of items on the Chronic Pain Self-Efficacy Scale reveals some overlap with the content of adjustment measures. For example, the self-efficacy measure contains the item, "How certain are you that you can perform your household chores" and the physical impairment measure contain the item, "During the past month, how much has your pain changed your ability to do household chores." Another example of similar content on the self-efficacy and adjustment measures is the item, "How certain are you that you can continue most of your daily activities" which is on the self-efficacy measure and the item, "During the past 
month, how much has your pain problem interfered with your day to day activities" which a physical impairment item. It is possible that patients respond to these items with similar content, in similar ways and that high correlations may be due to the similarity in the way the questions are asked. Fourth, patients may make more global judgements about self-efficacy than they do about specific coping behaviors. Self-efficacy beliefs may be more global, stable, and similar to personality dispositions than one's tendency to engage in particular coping behaviors. Unlike self-efficacy, the choice of coping behaviors may be more situation-specific or skill-dependent. In responding to items, it is possible that patients responded to questions about self-efficacy in a more global fashion, viewing themselves as either generally efficacious or generally nonefficacious, whereas, they may have made more subtle distinctions in responding to items about the use of specific coping techniques.

Emotional Factor and Adjustment

Results of this study fully supported the third hypothesis that emotional constructs would be associated with positive adjustment outcomes. Specifically, results of zero-order correlations between the emotional coping factor and outcomes suggest that patients with chronic myofascial pain who engage in more emotional coping report less sensory and affective pain, less physical impairment, and less depression. These results remained significant after 
controlling for sociodemographics with the exception of the relationship between emotional coping and physical impairment. Overall, results of these analyses suggest that patients who use more emotional coping and have greater emotional competencies have better adjustment outcomes. These patients report less pain intensity, less emotional distress due to their pain, and less depression. Thus, emotional coping appears to be a fairly robust predictor, especially for more subjective, rather than behavioral outcomes.

Although there is a significantly smaller research literature on emotional coping and adjustment outcomes in chronically-ill patients, the results of this study are consistent with previous findings. Overall, these studies have suggested that certain emotional coping styles are associated with favorable adjustment outcomes, particularly in chronic pain patients. As in this study, emotional confrontation, which is akin to emotional approach coping, was associated with positive psychological adaptation, and alexithymia was associated with negative psychological functioning in a sample of breast cancer patients (Paez et al., 1995). Similarly, Dominguez et al. (1995) found high levels of emotional and physical distress in chronic pain patients who were less competent in expressing and communicating their emotional states. Finally, as in this study, Jordan and Lumley (1993) found that alexithymic rheumatoid arthritis patients reported more depression and 
anxiety, less functional capacity, and less perceived pain control than nonalexithymic patients. It is notable that an interaction effect was found in this study such that patients who had high levels of alexithymia and who failed to use active cognitive-behavioral coping strategies demonstrated the highest levels of negative affect. Thus, this study lends additional support to the association between healthy emotional processing and better psychological and physical adaptation in chronically-ill patients.

Unique Predictive Abilities of Emotional coping Another hypothesis was that the emotional constructs would remain associated with adjustment after controlling for the cognitive constructs. This hypothesis was supported in part. Emotional Coping remained associated with both affective pain and depression when controlling for both the effects of both of the cognitive factors, Self-Efficacy and Cognitive Coping. Emotional Coping was unable to predict sensory pain beyond the effects of both cognitive factors together. This likely occurred because Emotional coping and Self-Efficacy have sufficient overlap that the relationship with sensory pain was eliminated. Emotional Coping was able to account for unique variance in sensory pain beyond the effects of Cognitive coping. In summary, even when the effects of cognitive factors were controlled, chronic myofascial pain patients who engage in more emotional coping experience less distress due to their pain and less 
depression. Further, when the effects of Cognitive Coping were controlled, these patients experience less pain intensity.

The results of this study provide some evidence that assessing emotional constructs, can add predictive power to cognitive constructs that have typically been used to predict adjustment outcomes in chronic pain populations. This is particularly true for affective outcomes that were more global in nature. Emotional coping did less well than Self-Efficacy in predicting the non-affective outcomes of sensory pain and physical impairment. Overall, the finding that Emotional Coping was able to predict unique variance in outcomes beyond the cognitive predictors is important in that it underscores the importance of assessing emotional processing, a task that has largely been ignored in the chronic pain literature.

The Unique Predictive Abilities of Cognitive Factors

Although the relative predictive power of cognitive factors was not the subject of a hypothesis in this study, it was an issue of interest. Thus, additional analyses were conducted that provided information about the ability of the two cognitive factors to predict beyond the effects of the emotion factor. Results of this study indicated that selfEfficacy remained a significant predictor of all of the outcome measures beyond the effects of Emotional Coping and sociodemographics. Thus, patients who have greater selfefficacy in regard to pain management, physical function, 
and coping with symptoms demonstrate better adjustment with less sensory and affective pain, less interference in daily activities due to pain, and less depression. On the other hand, Cognitive Coping could no longer predict affective pain and depression beyond the effects of the Emotional Coping factor and sociodemographics. These analyses suggest that Self-Efficacy is able to provide additional information beyond what is provided by Emotional Coping but the same does not hold true for Cognitive Coping which is a less robust predictor than both Self-Efficacy and Emotional Coping. It is likely that Emotional Coping predicts the outcomes of affective pain and depression better than Cognitive Coping because they are largely affective outcomes. Self-efficacy may be the best predictor of the three factors because it is a global characteristic but was measured in a way that it also made reference to pain. Relationships between Life Stress, coping, and Adjustment

Finally, it was hypothesized that stress would be inversely correlated with adjustment. In this study, this finding was only true of depression such that chronic myofascial pain patients with high levels of current life stress reported poorer affective functioning as indicated by higher levels of depression. This finding is fairly consistent with the literature in which current life stress has tended to be related to depression and other indices of negative psychological adjustment but not to pain-specific adjustment outcomes such as pain and physical impairment. 
For example, in a study with rheumatoid arthritis patients, Lumley et al. (1997) found that current life stress was related to affective disturbance but not to pain or physical dysfunction. Similarly, Kelley et al. (1997) used a disclosure intervention in order to attempt to reduce the negative effects of stress in a sample of rheumatoid arthritis patients. These researchers found that depression declined during the course of treatment but that pain was not affected.

In part, the lack of a significant relationship between life stress and pain-specific adjustment outcomes may be explained by the fact that life stress is fundamentally different than pain as a stressor. When patients report pain as their primary life stressor, they tend to report greater pain intensity; however, when patients report a nonpain stressor as their primary stressor, they do not report greater pain intensity (Turner et al., 1987). In this study, current life stress was assessed using a measure that largely tapped nonpain stressors such as divorce or death or a loved one rather than pain stressors. Thus, perhaps it is not surprising that stress was not found to be related to pain and physical impairment for this sample.

Another possible explanation for the nonsignificant relationship between life stress and pain-specific adjustment outcomes is that life stress does not have a direct effect on pain-specific adjustment outcomes. Instead, the effects of life stress on pain-specific 
outcomes may be weak because its effects are distal. For example, stress may cause negative affective changes, that subsequently could influence pain-specific outcomes. In this study as in other studies with chronic pain patients, current life stress was found to be related significantly to depression. Further, depression was found to be related significantly to sensory pain, affective pain, and physical impairment.

It also was hypothesized in this study that both cognitive and emotional constructs would correlate with adjustment beyond the effects of life stress. This hypothesis was fully supported. Because current life stress was not correlated significantly with sensory pain, affective pain, and physical impairment, of course, controlling for life stress did not change the relationships of the cognitive and emotional factors to those outcomes. When predicting depression, Self-Efficacy, Cognitive Coping, and Emotional Coping, all continued to predict unique variance in depression beyond the effects of life stress and sociodemographics. These findings demonstrate that selfEfficacy, Cognitive Coping, and Emotional Coping all add unique variance beyond life stress in the prediction of adjustment outcomes in chronic myofascial pain patients. This finding is consistent with studies in the recent pain coping literature. For example, in a study with rheumatoid arthritis patients, Lumley et al. (1997) found that emotional processing variables added to the prediction of 
pain, physical functioning, and psychological functioning in rheumatoid arthritis patients beyond that which was predicted by the experience of stressful events.

\section{Additional Findings}

Catastrophizing and disclosure were treated as separate predictors in this study. First, findings regarding catastrophizing will be discussed, followed by findings regarding disclosure. Numerous concerns about the Catastrophizing subscale of the Coping strategies Questionnaire have been raised by researchers in the chronic pain and coping literature (Geisser et al., 1994; Jensen et al., 1991). The Catastrophizing subscale measures patient's tendencies to engage in negativistic thinking and to worry in response in pain (Rosentiel \& Keefe, 1983). At times, catastrophizing has been treated as a measure of coping; however, this has been questioned because catastrophizing does not appear to fit well with commonly-used definitions of coping that involve purposeful efforts to deal with stress (Lazarus \& Folkman, 1984). Catastrophizing has been proposed as an appraisal or a belief about pain rather than an attempt to cope with it (Jensen et al., 1991).

Questions also have been raised about the conceptual overlap between catastrophizing and depression (Jensen et al., 1991; Sullivan \& D'Eon, 1990). Catastrophizing consistently has been found to demonstrate very large correlations with depression in previous studies. This also was true in this study in which catastrophizing and 
depression demonstrated a significant correlation of .69 . An examination of items on the Catastrophizing scale reveals that many of the items do appear to tap depression symptoms and depression-related cognitions. For example, the item "I feel like my life is not worth living" is similar to many items found on standard depression inventories that indicate depressive symptomatology. Other items such as "I feel like I can't go on" and "It's terrible and it's never going to get any better" appear to tap cognitions that have been closely linked to depression such as hopeless, global, stable, and internal attributions.

Also of interest in this study, catastrophizing demonstrated correlations of $\underline{r}=-.39$ to $\underline{r}=-.44$ with the Cognitive and Emotional Coping factors and a correlation of $\underline{I}=-.60$ with the Self-Efficacy factor, which taps beliefs or appraisals about one's pain. Past research has found that self-efficacy has been linked with positive adaptation and a wide variety of positive adjustment outcomes. In general, self-efficacy also appears to be a protective factor against psychopathology, especially depression with which it is negatively related. Thus, although catastrophizing initially was thought of as a predictor in this study, I would suggest that a better conclusion would be that it should be viewed as a measure of adjustment, similar to that of depression or negative affect. In support of this view, stanton et al. (1994) found that scales like catastrophizing on general coping measures 
typically are assessed by clinicians as psychopathology. Disclosure also was treated as a separate predictor in this study due to some issues regarding the measurement of disclosure. In this study, disclosure demonstrated small but statistically significant correlations with the selfEfficacy factor and with Interference. Thus, patients in this study who disclosed about life stressors to more individuals were found to have more self-efficacy in regard to their pain condition and to have less physical impairment than their counterparts who disclosed stressors to fewer others. This finding is consistent with the results of a study with rheumatoid arthritis patients in which disclosure demonstrated a marginally significant negative relationship with physical impairment; however in that study, disclosure also demonstrated a marginally significant negative relationship with pain and a significant negative relationship with affective disturbance, findings that did not emerge in the prior study (Lumley et al., 1997).

It was unclear whether disclosure should be placed with the emotional or cognitive predictors in this study. Unfortunately, the measure of disclosure that was utilized was not overly specific about the disclosure process. Thus, it was unclear whether individuals indicating that they engaged in more disclosure simply disclosed the facts of situations to others or whether they actively emotionally processed events by sharing their feelings. Thus, it appears that in this study, disclosure was operationalized 
poorly. Once disclosure scores were obtained, it also was unclear whether a mean level or absolute level of disclosure was more relevant. Thus, it could be that disclosure did not show many significant relationships with other variables due to measurement issues.

Another hypothesis is that disclosure may not have shown many relationships with adjustment outcomes because the relationship is not a direct one. For example, the relationship between disclosure and adjustment outcomes may be mediated by another intervening variable. One such mediating variable that has been suggested is ambivalence over emotional expression. King and Emmons (1990) proposed that ambivalence over emotional expression was an important mediator of the relationship between emotional processing styles and physical and psychological well-being. These researchers suggest that although individuals may have similar emotional expression styles, they may differ in the extent to which they feel conflicted over expressing their emotions. Further, this ambivalence may have important repercussions on one's health and well-being.

King and Emmons (1990) suggested that the current view that emotional expression is healthy and emotional inhibition is unhealthy is too simplistic. They proposed that emotional inhibition in and of itself may not be unhealthy, rather the important element in determining whether lack of expression is physically and psychologically detrimental is the extent to which one has a desire to 
express their emotions. Thus, congruence or incongruence between one's desire and one's actions is of significance with negative effects occurring in those who willfully prevent or actively inhibit themselves from disclosing when they have a desire to disclose. This suggests an interaction effect such that the effects of disclosure depend on how one feels about it. Disclosure that is ambivalent or forced may be unhealthy, just as disclosure that is emotionally neutral will not help. This is consistent with Pennebaker (1985) who indicated that it is active inhibition (e.g., voluntarily suppressing outward expression of emotions, purposely avoiding stimuli that produce a particular emotion, etc.) that leads to the negative effects of chronic physiological arousal and ruminative thoughts.

An alternative hypothesis for the lack of association between disclosure and adjustment outcomes is that disclosure taps some other construct or process entirely; one that was not measured in this study. For example, disclosure outside of laboratory situations typically involves sharing one's thoughts and/or feelings with another individual, thereby making it an interpersonal process. Although in this study, disclosure was viewed as an individually-based coping process whereby information is cognitively and affectively assimilated, it is possible that the more salient feature of disclosure as measured in this study involved the interpersonal transaction involved in 
disclosing stressful life events. In the studies by Pennebaker and colleagues, the interpersonal component of disclosure often was reduced or eliminated by having patients disclose by writing or talking into a tape recorder. In those situations, it is likely that the emotional and cognitive processing elements of the task superseded any interpersonal component. In the current study, disclosure was measured by self-report and the influence of social factors was not assessed. Thus, disclosure, as measured in this study, may be more highly related to measures of other interpersonal processes or constructs such as social networks or perceptions of social support. In summary, both poor measurement of disclosure and conceptual differences between disclosure and emotional and cognitive coping constructs are possible explanations for the dearth of significant relationships with adjustment outcomes in this study.

In order to examine differences in the prediction of adjustment constructs, the Emotional Approach Coping scale was modified with pain-specific instructions. Both measures were found to be highly correlated with each other. zeroorder correlations revealed that when compared to the Emotional Approach Scale with general instructions, the pain-specific version demonstrated correlations of comparable magnitude with all of the adjustment measures. Thus, no clear differences in prediction emerged using a pain-specific measure. This finding should be interpreted 
cautiously since psychometric properties have not been established for this measure. Further, it is likely that patients experienced some response bias in the way that they filled out the two questionnaires since only the directions for the measure were altered rather than the item content. Further studies using pain-specific measures of emotional coping will be necessary to determine whether there are significant differences in prediction using these measures. Finally, exploratory moderator analyses were conducted in this study. Results revealed that more frequent use of cognitive coping was associated with decreased sensory pain for men but not women, and for those with short pain durations but not for those with long pain durations. Similarly, more frequent use of cognitive coping was associated with decreased sensory pain and decreased physical impairment for patients in the low education group but not for those with higher educational levels. More frequent use of emotional coping was associated with decreased sensory pain for patients in the moderate education group but not for those in the low and high education groups. Finally, more frequent use of cognitive coping was associated with decreased affective pain in patients in the short pain duration group but not for those in the long pain duration group. Although this study provides some suggestion that these relationships exist, these relationships should be viewed with extreme caution due to the large number of analyses run and the increased 
probability of Type I error. Further studies with larger sample sizes will be necessary to replicate these findings.

\section{Implications}

Chronic myofascial pain is one of the primary types of chronic pain treated in pain clinics and primary health care settings. Psychological stress has been implicated as a major factor in the development, maintenance, and exacerbation of chronic myofascial pain syndromes. It appears that myofascial pain patients may have more of a tendency to respond to stress in a manner that results in tonic muscle contraction. Pain and stress exist in a vicious cycle for these patients. Stress results in chronic muscle tension that aggravates and activates existing trigger points causing pain and additional stress. Coping has been a factor that has been shown to significantly influence adjustment to chronic pain. Understanding how to mitigate the effects of stressors in chronic myofascial pain patients could result in significant benefits for this population. Thus, it is important to better delineate the specific coping strategies that are associated with positive adjustment in these patients. Past studies examining these issues have been plagued with conceptual and methodological problems. This study sought to examine the relationship between emotional and cognitive coping and adjustment in chronic myofascial pain patients using improved measures of cognitive and emotional constructs.

The major implication of this study is that there are 
two very important sets of variables relevant to the prediction of adjustment outcomes in a chronic myofascial pain population. Cognitive factors encompassing painspecific cognitive coping skills and pain-related selfefficacy beliefs, and an emotional factor comprised of emotional skills such as appropriately identifying, expressing, and regulating one's emotions were found to predict adjustment outcomes beyond the effects of life stress and sociodemographic variables. The two cognitive factors, Cognitive Coping and Self-Efficacy, and the emotional factor, Emotional Coping were found to be separate predictors, each accounting for unique variance in different domains of adjustment.

Most importantly, this study sought to introduce emotional processes and coping into the cognitive realm of stress, pain coping, and adjustment. Emotional processes including emotional coping largely have been overlooked in the chronic pain coping literature. In this study, Emotional Coping was found to be a predictor of most of the adjustment outcomes, particularly those related to subjective states such as pain and depression. Patients who frequently utilized Emotional Coping reported less sensory and affective pain, and less depression. Emotional Coping was found to be a better predictor of adjustment than Cognitive Coping and was able to predict several dimensions of adjustment, specifically affective pain and depression, beyond the effects of Self-Efficacy. An important 
implication of these findings is that Emotional Coping is healthy, adaptive, and associated with positive physical and psychological outcomes. Traditionally, emotion-focused coping has been viewed as pathological in the chronic pain literature and was associated with "getting overly emotional" or catastrophizing. Emotional Coping was abandoned in favor of rational, cognitive and behavioral methods of coping. This study highlights the fact that Emotional Coping can be adaptive and should be studied as such, rather than reflexively relegated to the status of pathology .

Few studies to date, have attempted to connect and consolidate research on individual emotional processes, coping styles, and dispositions. Another important implication of this study is that redundancy in measurement was identified among specific cognitive and specific emotional variables that have traditionally been researched separately. These variables were composited into a smaller number of meaningful factors. Now that these factors have been examined and associations with adjustment outcomes have begun to be identified, research in this area should continue to assess both cognitive and emotional variables, and attempt to determine their independent and joint contributions to adaptation.

This study was among the first to examine the role of emotional coping in patients with chronic health problems. This study provides additional evidence that emotional 
coping and emotional processing competencies are associated with positive adaptation, particularly in chronic myofascial pain patients. One major implication of this finding is that emotional coping processes might be incorporated into intervention studies with patients who have chronic myofascial pain conditions. These interventions could target improving emotional coping strategies in order to decrease the negative effects of pain and nonpain stressors on these patients. Psychological interventions with this population generally have been cognitive and behavioral in nature, emphasizing changing pain-related cognitions and teaching cognitive and behavioral coping skills. This study suggests that it likely would be adaptive to begin to supplement these interventions with those focused on teaching emotional competencies including such skills as regulating emotions; attending to and increasing awareness of emotions; identifying and distinguishing emotions from physiological sensations; describing and clarifying emotions; repairing negative emotions; and expressing emotions. Taylor et al. (1991) provide numerous examples of such emotion-focused interventions that have been used in working with alexithymic patients with somatoform disorders and medically-ill patients with alexithymic characteristics. In general, these approaches attempt to enhance awareness of and modify deficits in the way patients process and experience emotions.

Interventions focused on decreasing alexithymic 
characteristics might be particularly well-suited for chronic myofascial pain patients. Chronic pain patients generally have been found to be more alexithymic than individuals in the general population (Postone, 1986; Sririam et al., 1987; Taylor, 1984). Alexithymia has been linked with tonic, sympathetic arousal (Friedlander et al., 1997; Lumley et al., 1996). In turn, chronic sympathetic arousal has been associated with the maintenance and exacerbation of myofascial pain. Alexithymic chronic pain patients may be excessively attuned to their somatic sensations which could lead to the reporting of increased levels of pain and psychological distress - signs of poor adjustment. Thus, emotional coping interventions and affect-regulation strategies that address alexithymic characteristics including tonic sympathetic hyperarousal may be particularly beneficial for chronic myofascial pain patients.

\section{Limitations of the study}

This study has several limitations that should be considered. In this study, specific attention was given to choosing measures with strong psychometric properties. In addition, measures were chosen that addressed some of the methodological problems in the extant literature. Nonetheless, one of the limitations of this study is that it relied solely on uncorroborated, patient self-reports. Sole reliance on self-report measures can inflate validity coefficients due to shared method variance. Without 
measuring actual coping behavior, it is difficult to determine the validity of patient's self reports of their coping activities or adjustment outcomes, such as physical limitations. The inclusion of non-self-report methods to collect data likely would offer less biased estimates of the relationships among constructs measured in this study. Thus, in future studies, validity would be enhanced with the addition of more behavioral measures or by obtaining additional data from patient's family members or significant others to corroborate information provided by the patients. Unfortunately, there is not a commonly accepted measure of physical status for myofascial pain, as there are for other chronic pain conditions such as rheumatoid arthritis, nor are there reliable or valid physiological measures of pain intensity for this population. The coping constructs may be more amenable to behavioral measurement. For example, future studies may wish to utilize observations of what patients actually do to cope with their pain, using diary studies or random time sampling throughout the day. In terms of emotional coping, specific emotional skills tasks, such as those involving identifying and describing emotions, could be administered in order to further validate selfreports of emotional coping.

Another measurement limitation has to do with comparing generic and nonspecific measures of emotional coping with pain-specific measures of cognitive coping. This is problematic because measures of predictors that are highly 
specific to outcomes typically predict outcomes better than general measures of predictors. Research has shown that more specific measures of self-efficacy tend to be more highly predictive of adjustment outcomes than general measures. Thus, measures of pain-specific self-efficacy such as the Chronic Pain Self-Efficacy Scale (Anderson et al., 1995) and the Arthritis Self-Efficacy Scale (Lorig et al., 1989) were developed. Unfortunately, although a large number of pain-specific cognitive coping measures exist, at present, there are no pain-specific measures of emotional coping.

In this study, the Emotional Approach Coping Scale was modified to examine whether relationships with outcome measures were significantly enhanced as a function of using the pain-specific rather than general emotional coping measure; however, this measure's psychometric properties have not been evaluated, and response bias in filling out the two measures was very likely. As stated above, in general, it is expected that pain-specific measures would have more predictive power in predicting pain-specific outcomes, particularly sensory pain and physical impairment. This problem should be addressed in future studies by creating pain-specific emotional coping measures. Alternatively, more generic measures of cognitive coping could be added to future studies to allow for more direct comparisons with generic emotional measures in the prediction of pain adjustment. 
An additional measurement issue concerns the operationalization of disclosure. Disclosure was not operationalized well in this study; therefore, it was unclear exactly what disclosure was measuring. Further, the specific measure of disclosure that was used may have limited the relationships observed in this study. Certainly, ways of getting people to disclose emotionally stressful life events are available and have been utilized in other studies including those by Pennebaker and colleagues; however, it is unclear how to measure the extent to which patients disclose to others in the course of their daily lives. Depending on the research question, it may be possible to measure specific elements of disclosure by using the Pennebaker disclosure paradigm. For example, future studies could use indices such as number of emotion words expressed during the course of a disclosure session.

The cross-sectional, correlational design also imposes limitations on this study because cause-effect relations cannot be inferred. This study was one of the first to deal with emotional coping in a chronic pain population. This study was able to effectively reduce the number of emotional and cognitive variables to a more reasonable number by factor analyzing them to a smaller number before determining their relationships with adjustment outcomes in a chronic pain population. Although this was a necessary step, further studies will need to establish causal relationships between these factors and adjustment outcomes. Now that 
some preliminary relationships between emotional and cognitive factors and adjustment have been identified by this study, future studies should be more experimental in nature. The ultimate test of the importance of the emotional and cognitive variables in adjustment to chronic illness will be derived from controlled experimental or intervention studies, in which such variables are manipulated or changed, and the effects on adjustment are noted.

Generalizability of the results of this study is somewhat limited. First, the subject population for this study was not racially diverse due to the rural location from which subjects were drawn. Thus, results may not be generalizable to nonwhite patients or to those living in different geographic locations. Second, the results of this study may be generalizable only to pain patients with chronic myofascial pain syndromes and not to other chronic pain patient populations. Conversely, patients with myofascial pain syndromes often have additional chronic pain diagnoses such as mechanical back pain. One problem with research with this population is the difficulty of obtaining a pure sample of patients with only myofascial pain. Although attempts were made to recruit a relatively homogenous population, in this study, many patients did have additional pain diagnoses. For example, $25 \%$ of patients had an additional diagnosis of mechanical pain, 20\% had an additional diagnosis of arthritis, 15\% had an additional 
diagnosis of migraine headaches, and $7.5^{\circ}$ had an additional diagnosis of neuropathic pain. Although attempts were made to have patients answer questions in regard to their myofascial pain syndrome, it likely was difficult for patients to discriminate effects due to various pain conditions. Future studies may wish to address whether the findings of this study also apply to individuals with other pain conditions such as lower back pain or rheumatoid arthritis. Third, all patients in this study had obtained some type of treatment for their myofascial pain syndrome in the past. At the time of the study, $82.5 \%$ of patients actively were in treatment. Chronic pain patients who are recruited from clinic sites typically have more severe and refractory pain conditions. They tend to report more pain and physical dysfunction. This held for the subjects in this study who reported very high levels of pain. studies utilizing patients recruited from the community or other nonclinic locales may have produced significantly different results.

\section{Future Research Directions}

In addition to the improvements and suggestions for future research noted above, the results of this study also suggest several other directions for future research. First, future studies can begin to delineate situations in which patients rely on emotional versus cognitive coping techniques and to determine under which circumstances using a particular type of coping might be more adaptive. There 
is some research literature that suggests that patients may use more cognitive or problem-focused coping in situations that they view as more controllable or changeable, and more emotion-focused coping in situations in which they view the outcomes as being out of their control (Folkman \& Lazarus, 1988; Mattlin, Wethington, \& Kessler, 1990). Additional studies are needed to confirm this finding and to determine whether there are beneficial effects to the selection of particular types of coping under different circumstances.

An interesting issue that emerges when attempting to conduct research in the area of coping and adjustment concerns the level at which these constructs should be studied. It appears that the broadest level one can focus on is that of generalized health or generalized mental health. Healthy coping appears to account for much of the variance in adjustment outcomes. Subsumed under the level of generalized health is the level of second-order factors. This is the level at which this study was aimed. The results of this study suggest that there at least three second-order factors that can be subsumed under healthy coping: Emotional Coping, Self-Efficacy, and Cognitive Coping. In turn, each of these second-order factors subsumes many variables. For example, Emotional Coping appears to consist in part of alexithymia, emotional approach coping, and meta-mood skills. The level at which one studies these issues appears to be in part dependent on one's needs or the questions one wants to answer. For 
example, if one's aim is to globally categorize people into healthy versus unhealthy copers or to develop a basic screening tool to determine which patients are in need of psychological services, one may wish to focus at the broadest level of generalized healthy coping. In contrast, if one has an interest in developing a specific treatment program for patients, it may be necessary to focus on a lower-level of analysis. For example, if one wishes to identify particular emotional coping deficits in patients or to design treatment interventions aimed at improving Emotional Coping, it may be necessary to look at more specific facets or dimensions which underlie Emotional Coping. Thus, future studies in this area may need to focus on different levels of these constructs depending on their particular needs or the specific research questions to be answered.

In conclusion, this study was conducted to provide a better understanding of the relationships among coping and adjustment in chronic myofascial pain patients. In particular, this study sought to introduce emotional regulation to the realm of pain coping and adjustment. Further, this study sought to reduce the number of available cognitive and emotional predictors to a meaningful number of factors capable of predicting adjustment outcomes. Results of this study suggest that both cognitive and emotional factors are important in the prediction of physical and psychological adjustment outcomes in chronic myofascial pain 
patients. Self-Efficacy, Cognitive Coping, and Emotional Coping all were found to be separate predictors, each accounting for unique variance in different domains of adjustment. In particular, Emotional Coping was found to predict adjustment outcomes beyond the effects of life stress and to be a more robust predictor than Cognitive Coping. These findings underscore the importance of continuing to include emotional constructs in pain coping research and beginning to incorporate a focus on emotional coping strategies into psychological treatment interventions with this patient population. 
Appendix A

Informed Consent

175 

TO ADJUSTMISTT TO CHRONIC MYOFR.SCIAL PAIN

PRINCIPAI INVESTIGATOR: Julie A. Smith, M.A. CO-INVESTIGATOR: David J. Longo, Ph.D. PHONE NUMBER: (717) 271-6516 24 HOUR PHONE NUMBER: (717) 271-6211 (HOSPITAL SWITCHBOARD)

Chronic pain is a problem that has been associated with a variety of negative effects. Individuals who experience chronic pain often must cope with serious physical, emotional, social, and economic stressors. Individuals differ in the ways in which they cope with pain and other life stressors. The coping strategies one uses may be associated with important differences in the extent to which stress affects one's physical and emotional health. I am being asked to participate in a research study that will examine how I have been affected by the experience of pain and other life stressors and how I attempt to manage or cope with pain and stress. This study is being conducted at Geisinger Medical Center and will involve about 70 subjects. This research study will assess the relationship between coping strategies and a variety of physical and emotional outcomes in chronic pain patients.

If I choose to participate in the research study, I will be asked to complete several questionnaires about my health, pain, coping, and emotions. It will take about 90 minutes to complete the questionnaires. I understand that $I$ do not need to complete all the questionnaires in one sitting. I will be asked to mail the packet of questionnaires back to the investigators in the self-addressed envelope provided with the packet. All postage will be prepaid by the investigators. The principal investigator also will review my medical record to obtain information about my medical history such as treatment I have received for my pain. Information which identifies me will be kept in confidence to the extent permitted by law. All information about me will be kept in confidence. I will be identified only by a research number and all information I provide will be kept in a locked file cabinet. Accounts of this research that may be made known or published in the scientific literature will not identify me in any way.

I have read this consent form, or it has been read to me. I understand its contents and freely consent to participate in this study. I have received a copy of this consent form. I have had an opportunity to have all questions answered by the investigator.

STUDY SUBJECTS' SIGNATURE

WITNESS' SIGNATURE

\section{DATE}

DATE

DATE
INVESTIGATOR'S SIGNATURE 
Appendix B

Patient Information Form 


\section{PATIENT INRORMATION EORM}

Please provide the following information by filling in or circling the appropriate responses.

Sex

Age

Race

Work Status:

Employed Unemployed Retired Other

What is your occupation?

Are you currently receiving disability payments?

Are you currently receiving workman's compensation?

Relationship status:

Single/Never Married Married Divorced

Widowed Unmarried/Living with partner

Years of education: Circle the highest year completed.

$\begin{array}{lllllllllllll}1 & 2 & 3 & 4 & 5 & 6 & 7 & 8 & 9 & 10 & 11 & 12 & \text { years }\end{array}$

Associates Degree or Other Formalized Training

Bachelors Degree Masters Degree Doctoral Degree

Medical History:

How long have you experienced chronic pain?

Are you receiving medical treatment for your pain at this time? No Y Yes_ (circle what type below).

Physical therapy Occupational therapy Biofeedback.

Massage therapy Counseling/Therapy Medications

Stress Management Biofeedback Surgery

Steroid Injections Epidural Blocks Other 
Have you received medical treatment for your pain in the past? No Y_ Yes ___ (circle what type below).

Physical therapy Occupational therapy Biofeedback

Massage therapy Counseling/Therapy Medications

Stress Management Biofeedback Surgery

Steroid Injections Epidural Blocks Other

Please list any over-the-counter medications that you are taking for your pain?

Please list any prescription medications that you are taking for your pain?

Please list any other health problems that you have: 
PAIN DRAWING

Directions: Please mark the areas of your body where you feel the pain. Mark all affected body areas .with an ' $\underline{X}$ ' or other symbol
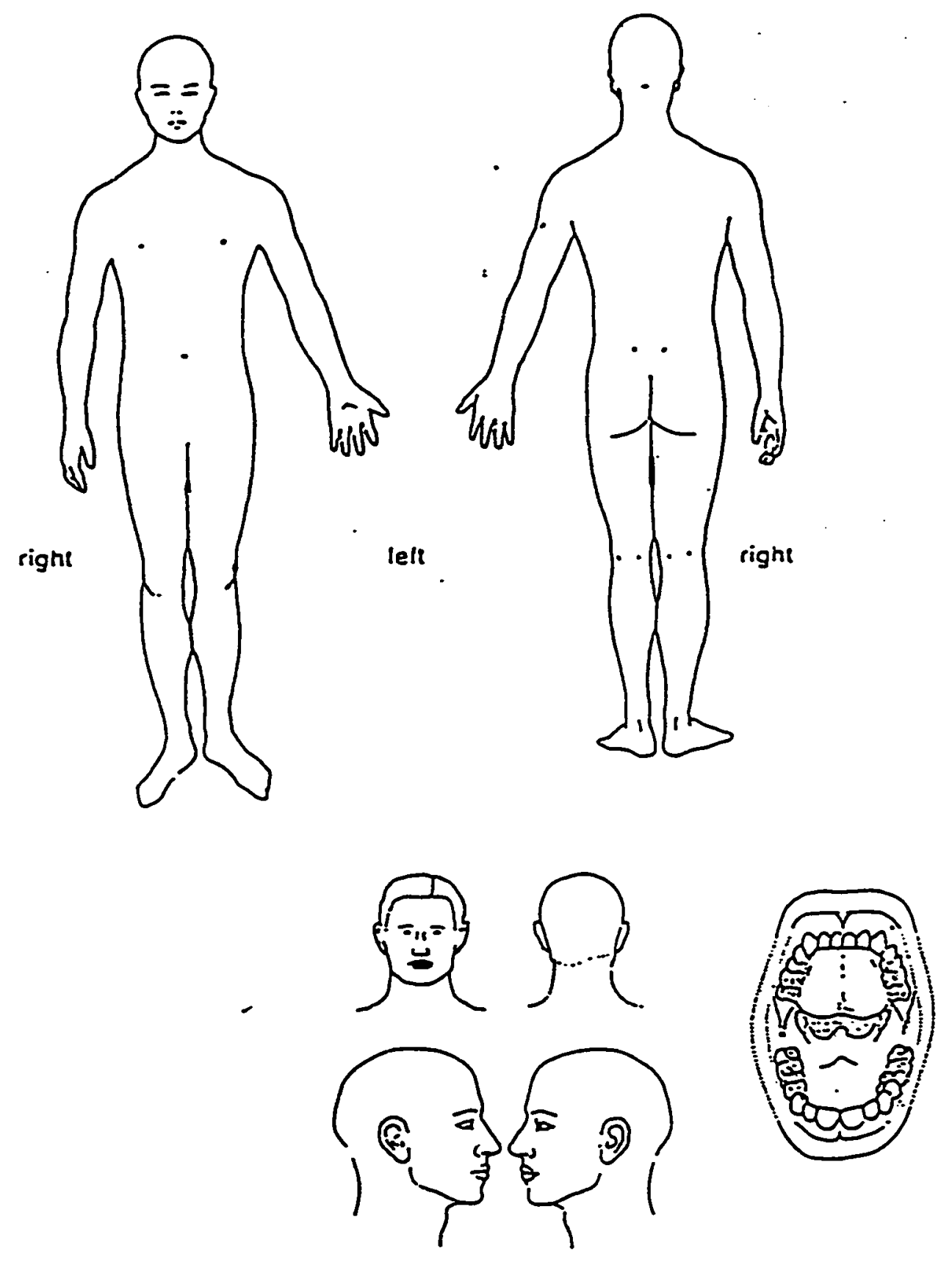
Appendix C

Vanderbilt Multidimensional Pain coping Inventory (VMPCI) 


\section{NOTE TO USERS}

Copyrighted materials in this document have not been filmed at the request of the author. They are available for consultation in the author's university library.

\section{Pages 182-185}

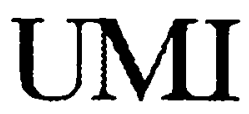




\section{Appendix D \\ Chronic Pain Self-Efficacy Scale (CPSS)}




\section{NOTE TO USERS}

Copyrighted materials in this document have not been filmed at the request of the author. They are available for consultation in the author's university library.

Pages 187-191

UMI 
Appendix E

Self-Control scale 


\section{NOTE TO USERS}

Copyrighted materials in this document have not been filmed at the request of the author. They are available for consultation in the author's university library.

Page 193

UMI 


\section{Appendix $\mathrm{F}$}

Twenty-Item Toronto Alexithymia scale (TAS-20) 


\section{NOTE TO USERS}

Copyrighted materials in this document have not been filmed at the request of the author. They are available for consultation in the author's university library.

\section{Page 195}

UMI 
Appendix G

Emotional Approach Coping Scale 


\section{NOTE TO USERS}

Copyrighted materials in this document have not been filmed at the request of the author. They are available for consultation in the author's university library.

Page 197

UMI 
Appendix $\mathrm{H}$

Trait Meta-Mood Scale - 30 Item Form (TMMS-30) 


\section{NOTE TO USERS}

Copyrighted materials in this document have not been filmed at the request of the author. They are available for consultation in the author's university library.

Pages 199-200

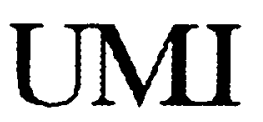


Appendix I

Life Events Checklist 


\section{NOTE TO USERS}

Copyrighted materials in this document have not been filmed at the request of the author. They are available for consultation in the author's university library.

\section{Pages 202-203}

UMI 
Appendix $\mathrm{J}$

Catastrophizing scale 


\section{NOTE TO USERS}

Copyrighted materials in this document have not been filmed at the request of the author. They are available for consultation in the author's university library.

Pages 205-206

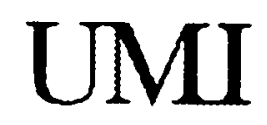


Appendix $\mathrm{K}$

Emotional Approach Coping scale - Pain Version 


\section{NOTE TO USERS}

Copyrighted materials in this document have not been filmed at the request of the author. They are available for consultation in the author's university library.

Page 208

UMI 
Appendix L

Pain Rating Index (PRI) of the MCGiIl Pain Questionnaire (MPO) 


\section{NOTE TO USERS}

Copyrighted materials in this document have not been filmed at the request of the author. They are available for consultation in the author's university library.

Page 210

UMI 
Appendix $M$

Visual Analouge Scales (VAS)

211 


\section{NOTE TO USERS}

Copyrighted materials in this document have not been filmed at the request of the author. They are available for consultation in the author's university library.

Page 212

UMI 
Appendix $\mathrm{N}$

Interference Scale 


\section{NOTE TO USERS}

Copyrighted materials in this document have not been filmed at the request of the author. They are available for consultation in the author's university library.

Pages 214-215

UMI 
Appendix 0

Center for Epidemiologic Studies - Depression Scale (CES-D) 


\section{NOTE TO USERS}

Copyrighted materials in this document have not been filmed at the request of the author. They are available for consultation in the author's university library.

\section{Page 217}

\section{UMI}


Appendix $\mathrm{P}$

Positive and Negative Affect Schedule - Expanded Form (PANAS - X) 


\section{NOTE TO USERS}

Copyrighted materials in this document have not been filmed at the request of the author. They are available for consultation in the author's university library.

\section{Page 219}

UMI 


\section{REFERENCES}

Anderson, K.O., Dowds, B.N., Pelletz, R.E., Edwards, W.T., \& Peeters-Asdourian, C. (1995). Development and initial validation of a scale to measure self-efficacy beliefs in patients with chronic pain. Pain, 63, 77-84.

Bagby, R.M., Parker, J.D.A., \& Taylor, G.J. (1994). The twenty-item Toronto Alexithymia Scale-I: Item selection and cross-validation of the factor structure. Journal of Psychosomatic Research, 38, 23-32.

Bagby, R.M., Taylor, G.J., \& Parker, J.D.A. (1994). The twenty-item Toronto Alexithymia Scale-II: Convergent, discriminant, and concurrent validity. Journal of Psychosomatic Research, 38, 33-40.

Bandura, A. (1977). Self-efficacy: Toward a unifying theory of behavioral change. Psychological Review, 84, 191215 .

Berenbaum, H., \& James, T. (1994). Correlates and retrospectively reported antecedents of alexithymia. Psychosomatic Medicine, 56, 353-359.

Blalock, S.J., DeVellis, B.M., Brown, G.K., \& Wallston, K.A. (1989). Validity of the Center for Epidemiological Studies Depression scale in arthritis populations. Arthritis and Rheumatism, 32, 991-997.

Blalock, S.J., DeVellis, B.M., \& DeVellis, R.F. (1989). Social comparison among individuals with rheumatoid arthritis. Journal of Applied Social Psychology, 19, 665680 .

Blumenthal, J.A. (1986). Relaxation therapies and biofeedback: Applications in medical practice. In J.L. Houpt, \& H.K.H. Brodie (Eds.), Psychiatry, Velume 3: consultation-liaison psychiatry and behavioral medicine. (pp. 273-285). Philadelphia: Lippincott.

Bonica, J.J. (1990a). Definitions and taxonomy of pain.

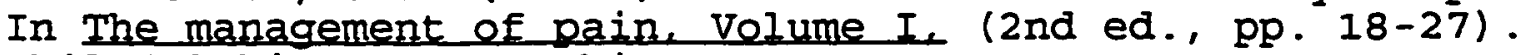
Philadelphia: Lea \& Febiger.

Bonica, J.J. (1990b). General considerations of chronic

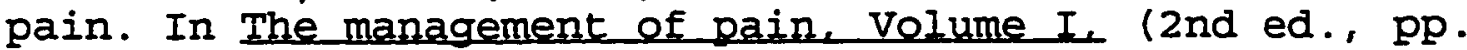
180-196). Philadelphia: Lea \& Febiger.

Brown, G.K. (1990). A causal analysis of chronic pain and depression. Journal of Abnormal Psychology, 92, 121-137.

Brown, G.K., \& Nicassio, P.M. (1987). Development of a questionnaire for the assessment of active and passive 
coping strategies in chronic pain patients. Pain, 31, 53-63.

Brown, G.K., Nicassio, P.M., \& Wallston, K.A. (1989). Pain coping strategies and depression in rheumatoid arthritis. Journal of consulting and Clinical Psychology, 57. 652-657.

Chapman, S.I. (1991). Chronic pain: Psychological assessment and treatment. In J.J. Sweet, R.H. Rozensky, \& S.M. Tovian (Eds.), Handbook of clinical psychology in medical settings (pp. 401-420). New York: Plenum Press.

Dolce, J.J., Crocker, M.F., Moletteire, C. \& \& Doleys, D.M. (1986). Exercise quotas, anticipatory concern and selfefficacy expectancies in chronic pain: A preliminary report. Pain, 24, 365-372.

Dominguez, B., Valderrama, P., Meza, M.A., Perez, S.L., Silva, A., Martinez, G., Mendez, V.M., \& Olvera, Y. (1995). The roles of disclosure and emotional reversal in clinical practice. In J.W. Pennebaker (Ed.), Emotion, disclosure, and health (pp. 255-270). Washington DC: American Psychological Association.

Esterling, B.A., Antoni, M.H., Fletcher, M.A., Margulies, S., \& Schneiderman. (1994). Emotional disclosure through writing or speaking modulates latent Epstein-Barr Virus antibody titers. Journal of Consulting and clinical Psychology, 62, 130-140.

Fernandez, A., Sriram, T.G., Rajkumar, S., Chandrasekar, A.N. (1989). Alexithymic characteristics in rheumatoid arthritis: A controlled study. Psychotherapy and esychosomatics, 44, 132-138.

Fernandez, E., \& Turk, D.C. (1989). The utility of cognitive coping strategies for altering pain perception: A meta-analysis. Pain, 38, 123-135.

Flor, H., \& Turk, D.C. (1988). Chronic back pain and rheumatoid arthritis: Predicting pain and disability from cognitive variables. Jounnal of Behavioral Medicine 11 251-265.

Folkman, S., \& Lazarus, R.S. (1988). Coping as a mediator of emotion. Journal of Personality and Social Psychology, 54, 466-475.

Francis, M.E., \& Pennebaker, J.W. (1992). Putting stress into words: The impact of writing on physiological, absentee, and self-reported emotional well-being measures. American Journal of Health Promotion, $6,280-287$.

Friedlander, L., Lumley, M.A., Farchione, T., \& Doyal, 
G. (1997). Testing the alexithymia hypothesis: Physiological and subjective responses during relaxation and stress. Journal of Nervous and Mental Disease, 185, 233-239.

Geisser, M.E., Robinson, M.E., \& Hensen, C.D. (1994). The Coping Strategies Questionnaire and chronic pain adjustment: A conceptual and empirical reanalysis. Clinical Journal of Pain, 10, 98-106.

Hall, R.C.W., \& Beresford. (1986). Psychiatric manifestations in physical illness. In J.L. Houpt, \& H.K.H. Brodie (Eds.), Psychiatry, Volume 3: Consultation-liaison psychiatry and behavioral medicine, (pp. 95-112). Philadelphia: Lippincott.

Horvath, T.B. (1986). Organic brain syndromes. In J.L. Houpt, \& H.K.H. Brodie (Eds.), Psychiatry, Volume 3: consultation-liaison esychiatry and behavieral medicine, (pp. 77-94). Philadelphia: Lippincott.

Jensen, M.P., \& Karoly, P. (1991). Control beliefs, coping efforts, and adjustment to chronic pain. Journal of consulting and clinical Psychology, 59, 431-438.

Jensen, M.P., \& Karoly, P. (1992). Pain-specific beliefs, perceived symptom severity, and adjustment to chronic pain. The clinical Journal of Pain, 8, 123-130.

Jensen, M.P., Turner, J.A., Romano, J.M., \& Karoly, P. (1991). Coping with chronic pain: A critical review of the literature. Pain, 47, 249-283.

Jordan, M.S., \& Lumley, M.A. (1993). Alexithymia and rheumatoid arthritis. Psychosomatic Medicine, $55,123$.

Karoly, P., \& Jenson, M. (1987). Multimethod assessment ef chronic pain. New York: Pergamon.

Keefe, F.J., Brown, G.K., Wallston, K.A., \& Caldwell, D.S. (1989). Coping with rheumatoid arthritis pain: Catastrophizing as a maladaptive strategy. Pain, 37, 51-56.

Keefe, F.J., Dunsmore, J., \& Burnett, R. (1992). Behavior and cognitive-behavioral approaches to chronic pain: Recent advances and future directions. Journal of consulting and Clinical Psychology, 60, 528-536.

Kelley, J.E., Lumley, M.A., \& Leisen, J.C.C. (1997). The health effects of emotional disclosure in rheumatoid arthritis. Health Psycholegy, 16, 331-340.

Kerns, R., \& Haythornthwaite, J. (1988). Depression among chronic pain patients: Cognitive-behavioral analysis and effects on rehabilitation outcome. Journal of consulting 
and Clinical Psychology, 56, 870-876.

Kerns, R.D., Turk, D.C., \& Rudy, T.E. (1985). The West Haven-Yale Multidimensional Pain Inventory (WHYMPI). Pain, 23, 345-356.

Kerns, R.D., Turk, D.C., \& Holzman, A. (1985). Comparison of cognitive-behavioral and behavioral approaches to the outpatient treatment of chronic pain. Clinical Journal of Pain, 1 , 195-203.

King, L.A., \& Emmons, R.A. (1990). Conflict over emotional expression: Psychological and physical correlates. Journal of Personality and Social Psychology, 58, 864-877.

Kores, R.C., Murphy, W.D., Rosenthal, T.L., Elias, D.B., \& North, W.C. (1990). Predicting outcome of chronic pain treatment via a modified self-efficacy scale. Behavior, Research, and Therapy, 28, 165-169.

Lazarus, R.S. (1993) . Coping theory and research: Past, present, and future. Psychosomatic Medicine, 55, 234-247.

Lazarus, R.S. , \& Folkman, S. (1984). Stress, appraisal, and coping, New York: Springer.

Lethem, J., Slade, P.D., Troupe, J.D.G., \& Bentley, G. (1983). Outline of a fear-avoidance model of exaggerated pain perception - I. Behaviour, Research, and Therapy, 21 , 401-408.

Longo, D., Messier, L., Nelson, R., Krishnan, K. , \& Alessi, T. (1998). Pain prediction as a function of coping style and primary stressor in chronic pain patients. Manuscript submitted for publication.

Lorig, K., Chastain, K.L., Ung, E., Shoor, S. , \& Holman, H.R. (1989). Development and evaluation of a scale to measure perceived self-efficacy in people with arthritis. Arthritis and Rheumatism, 32, 37-44.

Lumley, M.A., Asselin, L.A., \& Norman, S. (1997). Alexithymia in chronic pain patients. Comprehensive Psychiatry, 38, 160-165.

Lumley, M.A., Kelley, J.E., \& Leisen, J.C.C. (1997) . Predicting pain and adjustment in rheumatoid arthritis: The role of life stress and emotional processing. Journal of Health Psychology, 2, 255-264.

Lumley, M.A., Stettner, L., \& Wehmer, F. (1996). How are alexithymia and physical iliness linked? $A$ review and critique of pathways. Journal of Psychosomatic Research, 41 , 505-518. 
Mattlin, J.A., Wethington, E., \& Kessler, D.C. (1990). Situational determinants of coping and coping effectiveness. Journal of Health and Social Behavior, 31, 103-122.

Melzack, R. (1975). The McGill Pain Questionnaire: Major properties and scoring methods. Pain, 1, 277-299.

Melzack, R. \& Katz, J. (1992). The McGill Pain Questionnaire: Appraisal and current status. In D.C. Turk \& R. Melzack (Eds.), Handbook of pain assessment (pp. 152168). New York: Guilford Press.

Merskey, H., \& Bogduk, N. (Eds.).(1994). Classification of chronic pain: Descriptions of chronic pain syndromes and definitions of pain terms, second edition, seattle: IASP Press.

Mims, B.C. (1989). Sociologic and cultural aspects of pain. In C.D. Tollison (Ed.), Handbook of chronic pain management (pp. 17-25). Baltimore: Williams \& Wilkins.

Paez, D., Basabe, N., Valdoseda, M., Velasco, C., \& Iraurgi, I. (1995). Confrontation: Inhibition, alexithymia, and health. In J.W. Pennebaker (Ed.), Emotion, disclosure, and health (pp. 195-222). Washington DC: American Psychological Association.

Pennebaker, J.W. (1985). Traumatic experience and psychosomatic disease: Exploring the roles of behavioural inhibition, obsession, and confiding. Canadian Psychology, 26, 82-95.

Pennebaker, J.W. (1989). Confession, inhibition, and disease. Advances in Experimental Social Psychology, 22 , 211-244.

Pennebaker, J.W., \& BealI, S.K. (1986). Confronting a traumatic event: Toward an understanding of inhibition and disease. Journal of Abnormal Psychology, 95, 274-281.

Pennebaker, J.W., Colder, M., \& Sharp, L.K. (1990). Accelerating the coping process. Journal of Personality and Social Psychology, 58, 528-537.

Pennebaker, J.W., \& Francis, M.E. (1996). Cognitive, emotional, and language processes in disclosure. Cognition and Emotion, 10, 601-626.

Pennebaker, J.W., Hughes, C.F., \& O'Heeron, R.C. (1987). The psychophysiology of confession: Linking inhibitory and psychosomatic processes. Journal of Personality and social Psychology, 52, 781-793.

Pennebaker, J.W., Kiecolt-Glaser, J.K., Glaser, R. 
(1988). Disclosure of traumas and immune function: Health implications for psychotherapy. Journal of Consulting and clinical Psychology, 56, 239-245.

Petrie, K.J., Booth, R.J., Pennebaker, J.W., Davison, K.P., \& Thomas, M.G. (1995). Disclosure of trauma and immune response to a Hepatitis $B$ vaccination program. Journal of counsulting and Clinical Psychology, 63, 787-792.

Philips, H. (1988). Changing chronic pain experience. Pain, 32, 165-172.

Postone, N. (1986). Alexithymia in chronic pain patients. General Hospital Psychiatry, 8, 163-167.

Price, D.D., McGrath, P.A., Rafii, A., \& Buckingham, B. (1994). The validation of visual analouge scales as ratio scale measures for chronic and experimental pain. pain, 17 . 45-56.

Radloff, L.S. (1977). The CES-D scale: A self-report depression scale for research in general populations. Applied Psychological Measurement, $1,385-401$.

Romano, J., \& Turner, J. (1985). Chronic pain and depression: Does the evidence support a relationship. Psychological Bulletin, 97, 18-34.

Rosenstiel, A.K., \& Keefe, F.J. (1983). The use of coping strategies in chronic low back pain patients: Relationship to patient characteristics and current adjustment. Pain. 17, 33-44.

Salovey, P., \& Mayer, J.D. (1990). Emotional intelligence. Imagination, cognition, and personality, 9 , 185-211.

Salovey, P., Mayer, J.D., Goldman, S.L., Turvey, C., \& Palfai, T.P. (1995). Emotional attention, clarity, and repair: Exploring emotional intelligence using the Trait Meta-Mood Scale. In J.W. Pennebaker (Ed.), Emotion, disclosure, and health (pp. 125-154). Washington DC: American Psychological Association.

Simons, D.G. (1989). Single-muscle myofascial pain syndromes. In C.D. Tollison (Ed.), Handbook of chronic pain management (pp. 490-508). Baltimore: Williams \& Wilkins.

Simons, D.G., \& Simons, I.S. (1989). Chronic myofascial pain syndrome. In C.D. Tollison (Ed.), Handbook of chronic pain management (pp. 509-529). Baltimore: Williams \& Wilkins.

Smith, C.A. \& Wallston, K.A. (1996). An analysis of 
coping profiles and adjustment in persons with rheumatoid arthritis. Anxiety, Stress, and Coping, 9, 107-122.

Smith, C.A., Wallston, K.A., Dwyer, K.A., \& Dowdy, S.W. (1997). Beyond good and bad coping: A multidimensional examination of coping and pain in persons with rheumatoid arthritis. Annals of Behavioral Medicine, 19, 11-21.

Smith, T.W., Peck, J.R., Milano, R.A., \& Ward, J.R. (1988). Cognitive distortion in rheumatoid arthritis: Relation to depression and disability. Journal of Consulting and Clinical Psychology, 56, 412-416.

Smith, T.W., Follick, M.J., Ahern, D.K., \& Adams, A. (1986). Cognitive distortions and disability in chronic low back pain. Cognitive Therapy and Research, 10, 201-210.

Sola, A.E., \& Bonica, J.J. (1990). Myofascial pain syndrome. In J.J. Bonica, The management of pain, volume I (2nd ed., pp. 352-367). Philadelphia: Lea \& Febiger.

Spera, S.P., Buhrfeind, E.D., \& Pennebaker, J.W. (1994). Expressive writing and coping with job Ioss. Academy of Management Journal, 37, 722-733.

Sriram, T.G., Chaturvedi, S.K., Gopinath, P.S., \& Shanmugam, V. (1987). Controlled study of alexithymic characteristics in patients with psychogenic pain disorder. Psychotherapy and Psychosomatics, 47, 11-17.

Stanton, A.I., Danoff-Burg, S., Cameron, C.I., \& Ellis, A.P. (1994). Coping through emotional approach: Problems of conceptualization and confounding. Journal of Personality and Social Psychology, 66, 350-362.

Stein, M., Shleifer, S.J., \& Keller, S.E. (1986). Brain, behavior, and immune processes. In J.I. Houpt, \& H.K.H. Brodie (Eds.), Psychiatry, Volume 3 ; Consultationliaison psychiatry and behavioral medicine, (pp. 401-419). Philadelphia: Lippincott.

Sternbach, R.A. (1990). Psychophysiologic pain syndromes. In J.J. Bonica, The management of pain. Volume I (2nd ed., pp. 287-290). Philadelphia: Lea \& Febiger.

Sullivan, M., \& D'Eon. J. (1990). The relationship between catastrophizing and depression in chronic pain patients. Journal of Abnormal psycholegy, 99, 260-263.

Taylor, G.J. (1984). Alexithymia: Concept, measurement, and implications for treatment. American Journal of Psychiatry, 141, 725-732.

Taylor, G.J., Bagby, R.M., \& Parker, J.D.A. (1991). The 
alexithymia construct: A potential paradigm for psychosomatic medicine. Psychosomatics, 32, 153-164.

Turk, D.C., \& Rudy, T.E. (1988). Toward an empirically derived taxonomy of chronic pain patients: Integration of psychological assessment data. Journal of consulting and Clinical Psychology, 56, 233-238.

Turk, D.C., \& Rudy, T.E. (1989). A cognitive-behavioral perspective on chronic pain: Beyond the scalpel and syringe. In C.D. Tollison (Ed.), Handbook of chronic pain management (pp. 222-236). Baltimore: Williams \& Wilkins.

Turk, D.C., \& Rudy, T.E. (1991). Neglected topics in the treatment of chronic pain patients - relapse, noncompliance, and adherence enhancement. Pain, 44, 5-28.

Turner, J.A., Clancy, S., \& Vitaliano, P.P. (1987) . Relationships of stress, appraisal, and coping to chronic back pain. Behaviour Research, and Therapy, 25, 281-288.

Watson, D. (1988). The vicissitudes of mood measurement: Effects of varying descriptors, time frames, and response formats on measures of positive and negative affect. Journal of Personality and Social Psychology, 55 . 128-141.

Watson, D., \& Clark, L.A. (1994). The PANAS-X: Manual for the Positive and Negative Affect Schedule - Expanded Form. The University of Iowa.

Watson, D., Clark, L.A., \& Tellegen, A. (1988). Development and validation of a brief measure of Positive and Negative Affect: The PANAS Scales. Journal of Personality and Social Psychology, 54, 1063-1070.

Watson, F., \& Pennebaker, J.W. (1989). Health complaints, stress, and distress: Exploring the central role of negative affectivity. Psychological Review, 96, 234-254.

Wilkie, D.J., Savedra, M.C., Holzemer, W.L. Tesler, M.D. \& Paul, S.M. (1990). Use of the McGill Pain Questionnaire to measure pain: A meta-analysis. Nursing Research, 39, 36-41. 


\begin{abstract}
THE RELATIONSHIP OF COGNITIVE AND EMOTIONAL COPING TO ADUUSTMENT TO CHRONIC MYOEASCIAL PAIN
\end{abstract}

by

JULIE ANN SMITH

December 1998

Advisor: Mark A. Lumley, Ph.D.

Major: Psychology (Clinical)

Degree: Doctor of Philosophy

In the chronic pain literature, pain coping and adjustment traditionally have been studied using cognitive and behavioral constructs. Little research attention has been devoted to exploring the role of emotional factors and their contribution to adjustment in chronic pain patients. Recent empirical evidence suggests that certain emotional approach coping strategies and competencies are associated with positive adaptation. This study was conducted to provide a better understanding of the relationship between cognitive and emotional coping and adjustment in chronic myofascial pain patients. In particular, this study sought to introduce emotional processes into the pain coping and adjustment 1iterature. Cognitive and emotional models were compared with each other and with the influence of life stress, in the prediction of adjustment. study participants were 80 adults with chronic myofascial pain syndromes. Patients completed self-report measures of cognitive constructs (self-efficacy and cognitive pain coping 
strategies), emotional constructs (emotional approach coping, alexithymia, and meta-mood skills), life stress, and adjustment. Healthy adjustment was conceptualized as low levels of sensory and affective pain, physical impairment, and depression. Results of this study revealed that both cognitive and emotional factors were important in the prediction of physical and psychological adjustment outcomes in chronic myofascial pain patients. When factor analyzed, emotional constructs loaded together on one Emotional Coping factor. Cognitive constructs loaded onto two factors, SelfEfficacy and Cognitive Coping. An overall factor analysis including emotional and cognitive constructs produced a three factor solution. Self-Efficacy, Cognitive Coping, and Emotional Coping all were found to be separate predictors, accounting for unique variance in different domains of adjustment and predicting adjustment beyond the effects of life stress. Self-Efficacy was found to be a particularly robust predictor of positive adaptation. Emotional Coping was found to be a more robust predictor than Cognitive Coping. Both cognitive and emotional constructs play a key role in understanding adjustment in chronic myofascial pain patients. Further, assessing emotional constructs can add predictive power to cognitive constructs typically used to predict adaptation to chronic pain. 


\section{AUTOBIOGRAPHICAL STATEMENT}

\section{Julie Ann Smith}

Julie Smith was born and raised in a suburb of Chicago, Illinois. She attended Nazareth Academy High School in LaGrange Park, IL. She received her B.S. from Loyola University of Chicago in 1990. She graduated Summa Cum Laude with an Honors degree in Psychology. Julie earned an M.A. in Clinical Psychology in 1995 and $a$ Ph.D. in Clinical Psychology in 1998 from Wayne state University in Detroit, Michigan. In 1996-7, she completed her predoctoral internship in Adult Clinical Psychology and Behavioral Medicine at Geisinger Medical Center in Danville, Pennsylvania. She now Iives in Reisterstown, Maryland and is a Linda Pollin Foundation Postdoctoral Fellow in Medical Crisis Counseling at the University of Maryland Medical Center in Baltimore.

Julie worked in a wide variety of clinical settings as part of her clinical training. She completed clinical practica in Detroit, MI at wayne state University Psychology Clinic, Wayne County Probate Court - Clinic for Child study, Children's Center of Wayne County, and Wayne state University School of Medicine - University Psychiatric Center. She served as a Clinic Co-Director and Coordinator of Therapy and Assessment Services at the Wayne state University Psychology Clinic. In addition to her clinical training experiences, she also provided clinical and research services at Childrens' Memorial Hospital (Chicago, IL), Michigan Health Care Corporation's Aurora Hospital for Children and Adolescents (Detroit, MI), Wayne state University School of Medicine - University Health Center (Detroit, MI), and Penn State Geisinger Health System Geisinger Medical Center (Danville, PA).

Julie has a broad range of psychology interests. Her primary focus is medical psychology including consultationIiaison, women's health issues, and emergency services. Additional psychotherapy interests include eating disorders, abuse issues, and personality disorders. Her research interests are in the areas of health psychology, adult attachment, and emotional processes. Her research publications and presentations have dealt with subjects including regimen adherence in diabetic adolescents, risk factors for substance use, and metacognition.

In her spare time, Julie enjoys being an aunt to her nephew, Zachary; playing with her two cat companions, Max and Alexandra; reading; spending time with friends and family; seeing movies; dining at ethnic restaurants; travelling; spending time in nature; surfing the Internet; and learning more about cultural anthropology and sociobiology. 